\title{
Vibration Signature Analysis for Gearbox Spalling Detection
}

\author{
by \\ Weidong Li
}

A thesis submitted to the Faculty of Graduate and Postdoctoral Affairs in partial fulfillment of the requirements for the degree of

Master of Applied Science

in

Mechanical Engineering

Carleton University

Ottawa, Ontario

(C) 2016, Weidong Li 


\begin{abstract}
Condition monitoring of gearboxes has been increasingly demanded in machinery maintenance. Many research efforts have been devoted to developing efficient and effective techniques for gear spalling detection. Most of them rely on predefined spalling and results are not representative in particular cases. This thesis presents a progressive development of the techniques to detect an irregular spall in a gearbox. Results show that well-adapted techniques such as amplitude demodulation and wavelet map are prominent in describing the amplitude modulation caused by a spall. Phase demodulation indicates the phase variation introduced by a spall. A fault indicator based on the ratio of spectral power is proposed to monitor the development of the spalling. An enhanced empirical mode decomposition (EMD) and Teager's Kaiser Energy Operator (TKEO) is developed to effectively extract the critical characteristics of spalling diagnosis. The relation between the digital signatures and the physical state of gear is comprehensively discussed.
\end{abstract}




\section{Acknowledgements}

First and foremost, I offer my sincerest gratitude to my supervisor Dr. Jie Liu, who has offered me the opportunity to participate in the graduate study while providing guidance and encouragement throughout this project. I would also like to express my sincere appreciation to my co-supervisor, Dr. Azzedine Dadouche for his effort in helping me solve technical problems and overcome personal difficulties and I am grateful for his support on completing the experiments and finishing the research.

I am thankful to the National Research Council Canada providing me with access to its facilities and equipment to complete the experiments and research work. I also thank Department of National Defence for the funding the experimental part of this research.

I would like to thank an earnest technician, Mr. Randy Payette, who has helped me to set up the test rig in the laboratory and passed me invaluable experience on machines and electric equipment.

My deepest gratitude goes my parents who have devoted their time, efforts and patient to my master study. They never force me to do things I don't like and always offer me full support on my decision. It makes my life full of freedom and creativity as well as a challenge. 
To My Extraordinary Parents 


\section{Table of Contents}

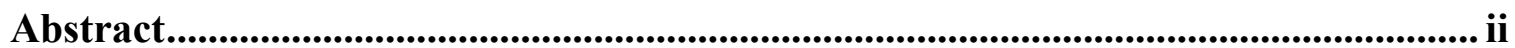

Acknowledgements .................................................................................................ii

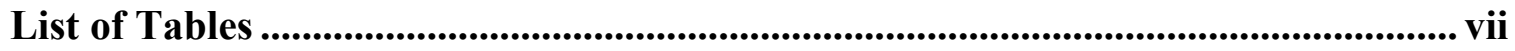

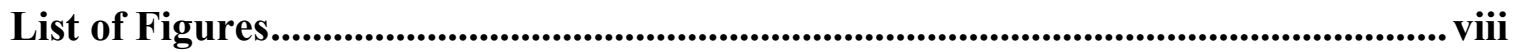

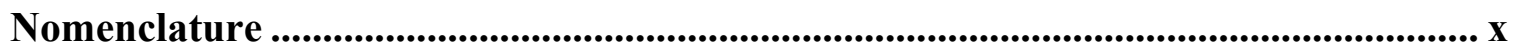

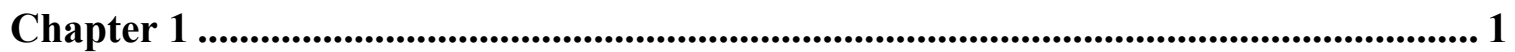

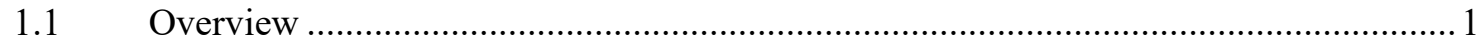

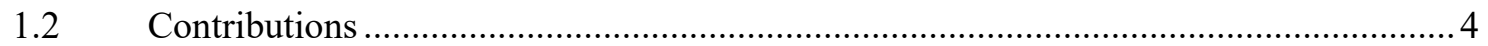

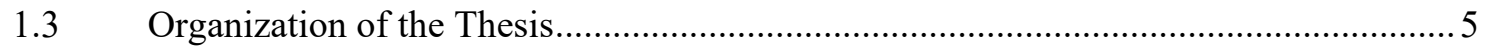

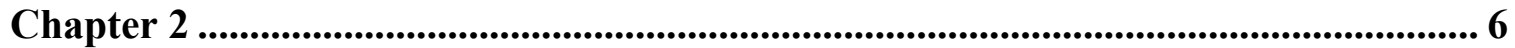

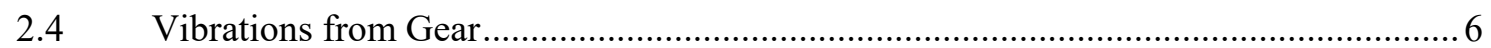

2.5 Gear Fault Detection Techniques Based on Vibration Signals ................................. 8

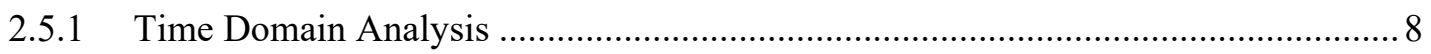

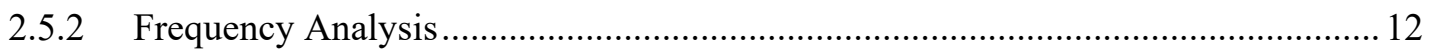

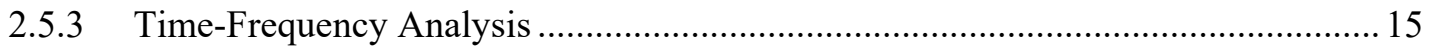

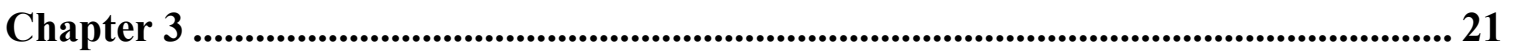

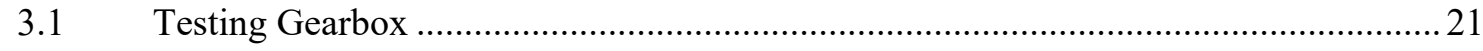

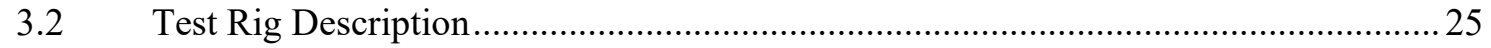

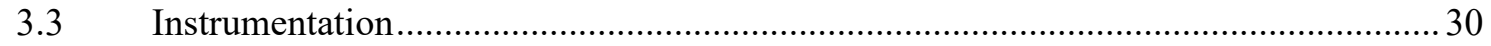

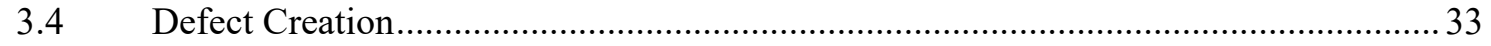

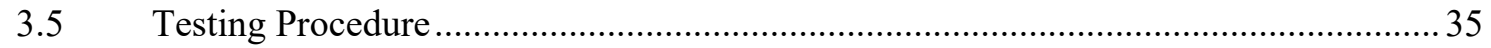

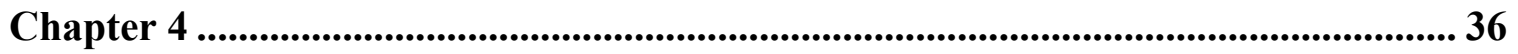




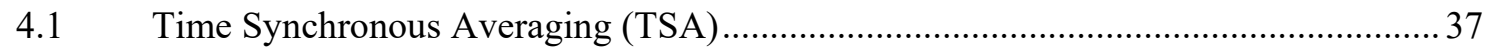

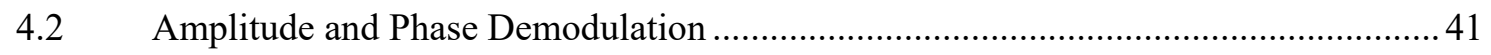

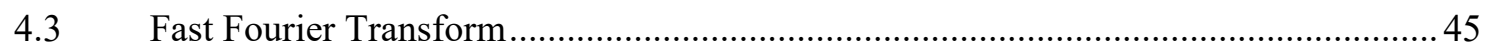

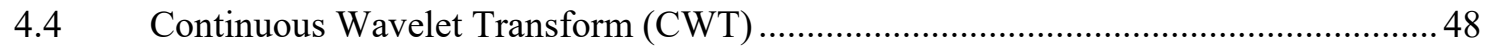

4.5 Enhanced Empirical Mode Decomposition (EMD) and Teager's Kaiser Energy

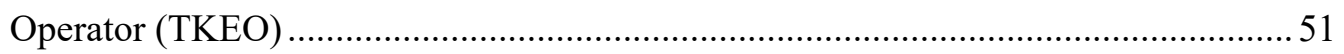

Chapter 5 ................................................................................................................................... 65

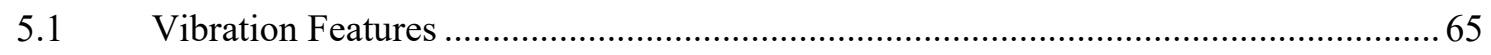

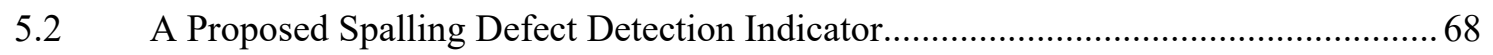

5.3 Gear Assembly Phase Frequency (GAPF) …..................................................... 71

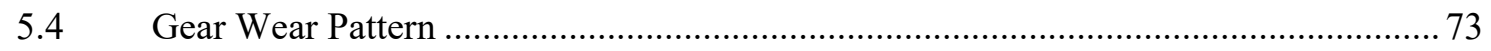

5.5 Vibration Signatures as Results of Gear Physical State ............................................ 76

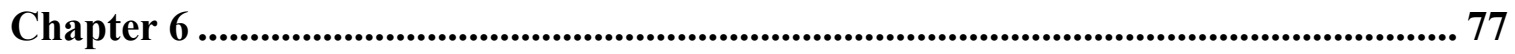

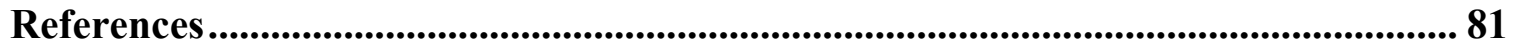




\section{List of Tables}

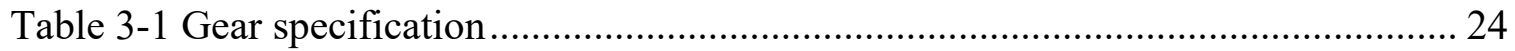

Table 3-2 Rotation frequencies of shafts and gear meshing frequency ......................... 24

Table 5-1 Magnitude ratio of recorded data ................................................................ 70 


\section{List of Figures}

Figure 1-1 A schematic of a typical spall and pit [3] ................................................. 3

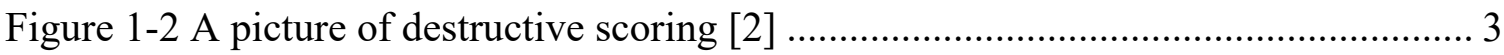

Figure 3-1 General Electric J85 engine and its accessory gearbox [79] ......................... 23

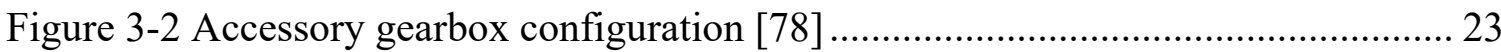

Figure 3-3 Schematic of the accessory gearbox and attached mechanical accessories [78]

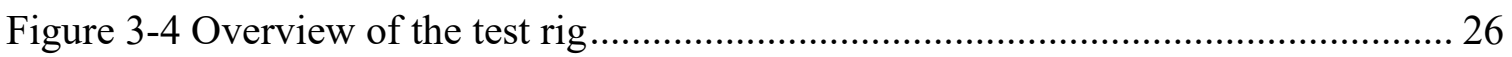

Figure 3-5 Schematic of lubrication system: a. original, b. modified [78] ...................... 29

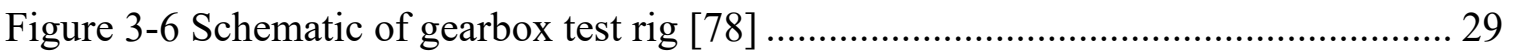

Figure 3-7 Gearbox condition monitoring sensors …………………......................... 30

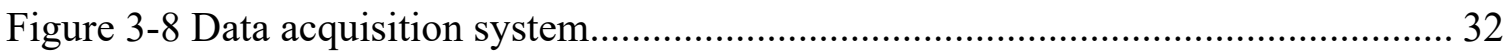

Figure 3-9 Tachometer coupled to the gearbox ………………..................................... 32

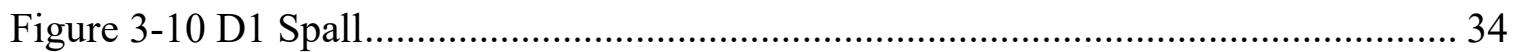

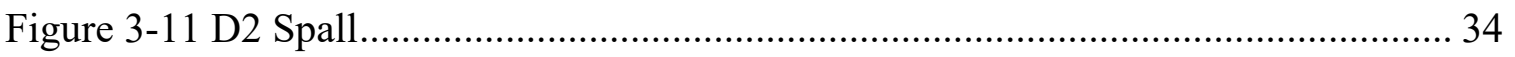

Figure 4-1 (a) Raw Signal (b) TSA signal................................................................ 39

Figure 4-2 TSA signal at applied load \#3 of 1) Baseline test, 2) D1 test, 3) D2 test........ 40

Figure 4-3 Amplitude Demodulation of TSA signals ...................................................... 44

Figure 4-4 Phase demodulation of TSA signals ........................................................... 44

Figure 4-5 TSA spectrum: 1) baseline test, 2) D1 test, 3) D2 test................................. 47

Figure 4-6 Wavelet map of TSA signals: 1) baseline test, 2) D1 test, 3) D2 test............. 50

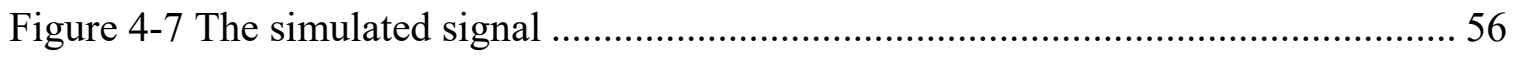

Figure 4-8 End effect illustration. Envelopes 1) without extrapolation 2) with 
extrapolation

Figure 4-9 EMD decomposition of the simulated signal ............................................... 58

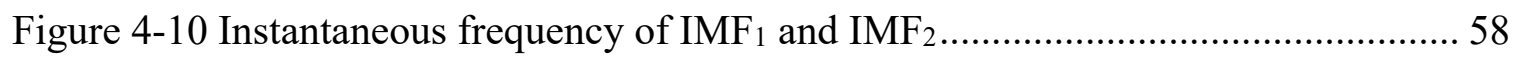

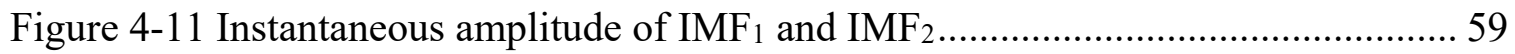

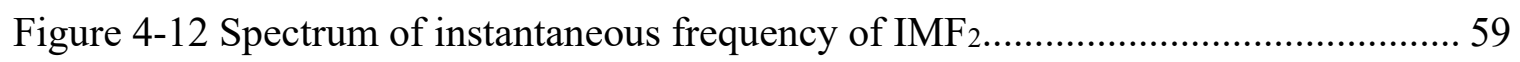

Figure 4-13 The first 4 IMFs of baseline TSA EMD decomposed signal ......................... 61

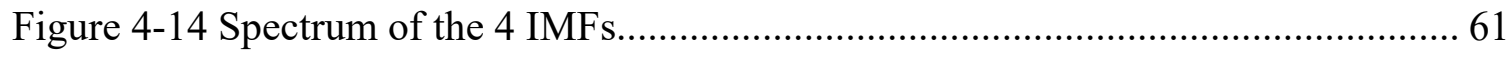

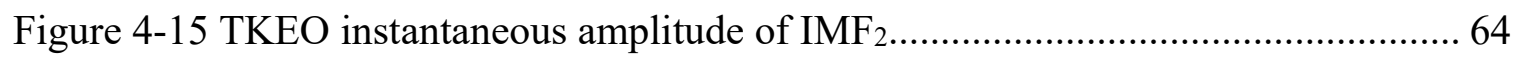

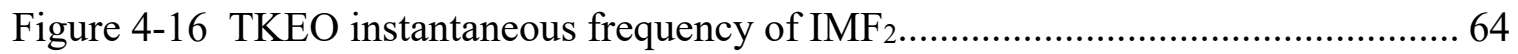

Figure 5-1 TSA signal segments overlap 1) Baseline test, 2) D1 test, 3) D2 test............. 67

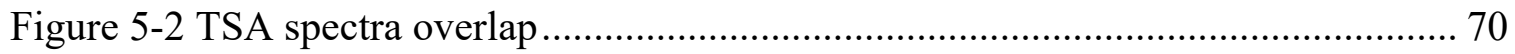

Figure 5-3 A schematic of gear F2 and gear C .......................................................... 72

Figure 5-4 The conditions of the 24 teeth of gear F2. …………….......................... 75

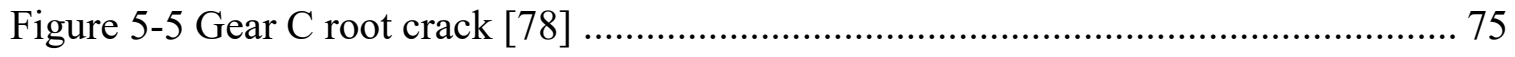




\section{Nomenclature}

EMD: Empirical Mode Decomposition

TKEO: Teager's Kaiser Energy Operator

GMF: Gear Mesh Frequency

RMS: Root Mean Square

TSA: Time Synchronous Averaging

NBD: Narrow-Band Demodulation

STFT: Short Time Fourier Transform

CWT: Continuous Wavelet Transform

DWT: Discrete Wavelet Transform

DTCWT: Dual-Tree Complex Wavelet Transform

IMF: Intrinsic Mode Functions

EEMD: Ensemble Empirical Mode Decomposition

VF: Vertical Front (accelerometer)

VB: Vertical Back (accelerometer)

HR: Horizontal (accelerometer)

ACUT: Air-Coupled Ultrasonic Transducer

AE: Acoustic Emission

D1: Defect \#1 (Spalling with size 1)

D2: Defect \#2 (Spalling with size 2)

FFT: Fast Fourier Transform

GAPF: Gear Assembly Phase Frequency

MR: Magnitude Ratio 


\section{Chapter 1}

\section{Introduction}

\subsection{Overview}

Machinery maintenance is an important action in the industry world, which 'cures the diseases' within machines or plants. It ensures the productivity, reliability, high performance and safety of machines. Inefficient maintenance procedures or improper maintenance schedule will increase maintenance cost and extend machine downtime or could cause serious machine failures. Gears represent one of the most critical components of various rotating machinery, especially in aircraft accessory gearboxes. Malfunction of gearboxes could be caused by unexpected gear damages and may eventually lead to sudden machinery failures. Thus, monitoring the condition of gearboxes is necessary for effective maintenance and preventing catastrophic machine damage. Condition-based maintenance is widely used in machinery maintenance since it gives high accuracy on fault diagnostics and high efficiency on maintenance procedures. The most common methodology of condition-based monitoring is accomplished through vibration-based analysis which has been well established for gearbox fault detection. The vibration-based analysis is performed on vibration signals where feature extraction techniques are extensively developed. Abnormal operation of a gearbox is usually a sign of gear failure. Common gear failures can be categorized into distributed and localized defects [1]. Distributed gear 
fault often refers to teeth surface wear and localized defects including pitting, spalling, scoring and tooth breakage [2]. The causes of gear failures can be summarized into internal factors of gears and external effects. Internal factors are gear material fatigue and subsurface crack development. On the other hand, external effects involve inadequate lubrication, contamination, gearbox overloaded and gear misalignment. This thesis focuses on spalling detection of gearboxes as well as teeth wear diagnosis.

Spalling is a common incipient gear failure due to surface contact fatigue under normal operating condition. According to [2], the formation of ordinary spalling is the development of pitting redistribution. Initial pitting occurs when teeth surface contact stresses reach the fatigue limit of the gear material. Then more and larger pits appear on the teeth surface as the same amount of load is distributed across the unpitted area. Pitting continues to aggravate until the edge of adjacent pits break away and large irregular craters are formed. Recent research provides primary descriptions of the difference between the developments of pitting and spalling [3]. The latter article points out that pitting is developed from surface defects whereas spalling mainly develops from subsurface cracks. As shown in Figure 1-1, a spalling appears as an irregular cavity at a tooth surface near the pitch line of a depth of 20-100 $\mu \mathrm{m}$. On the contrary, a common pitting covers only onethird area of a typical spalling and its depth is usually less than $10 \mu \mathrm{m}$.

Gear scoring is the continuous development of gear tooth wear and is the result of high operation temperature and pressure when gears are overloaded [2]. A picture of a gear with destructive soring is given in Figure 1-2. Inadequate lubrication, high operation temperature or pressure generate excessive heat that leads to local lubricant film breakdown and causes substantial sliding. It allows the mating gears metal-to-metal contact 
and forms welding which appears as scratch lines in the direction of sliding near addendum or dedendum.

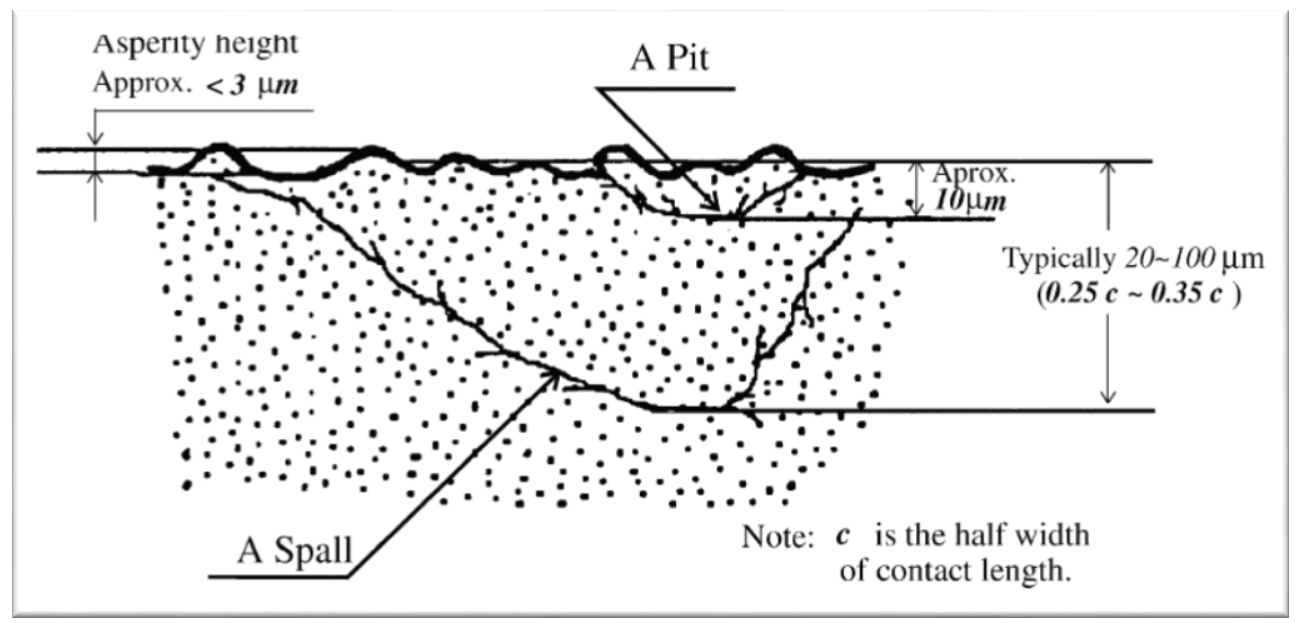

Figure 1-1 A schematic of a typical spall and pit [3]

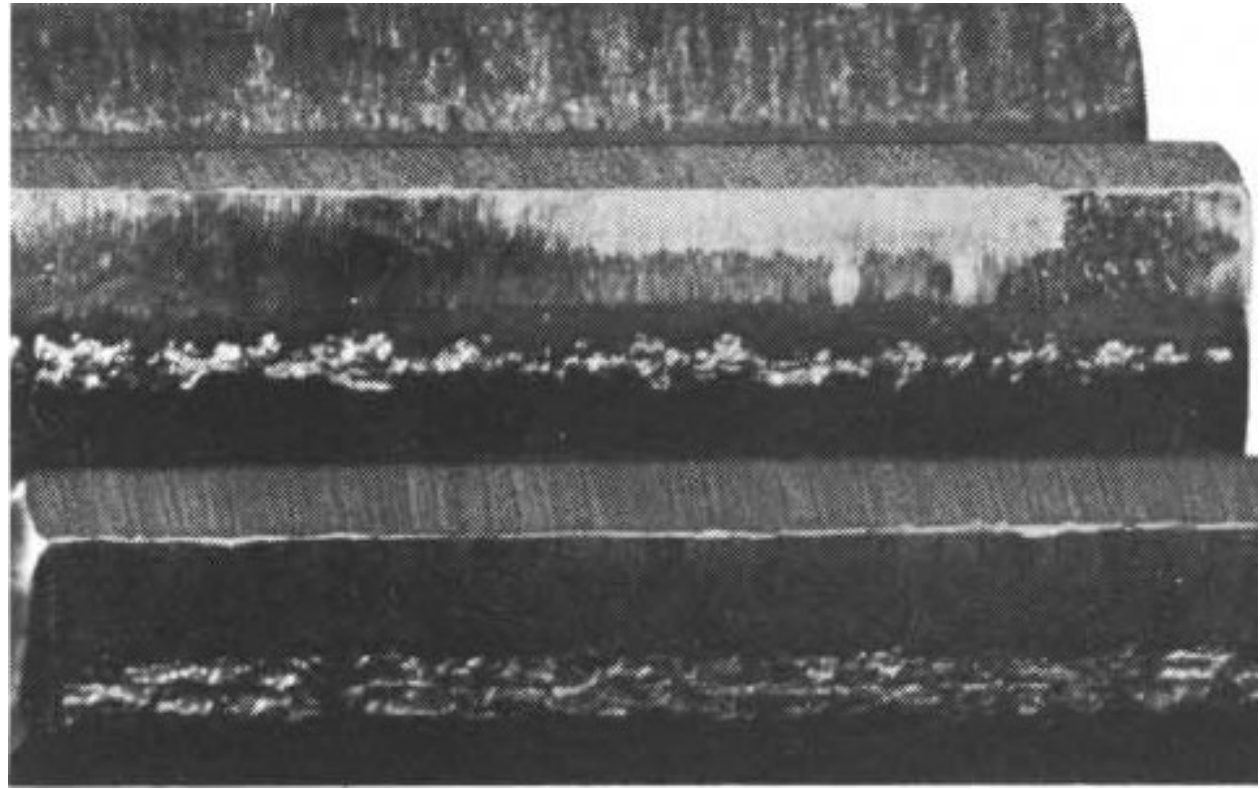

Figure 1-2 A picture of destructive scoring [2] 


\subsection{Contributions}

This research project aims at contributing to the field of vibration-based gearbox fault detection and developing reliable and robust analysis techniques applied to gearbox health management. The main contributions are described below:

1. Design and creation of a spalling defect on an aircraft accessory gearbox and run experiments simulating the operating environment on an aircraft engine.

2. Evaluation of the performance of commonly adopted signal processing techniques in extracting defect related features from vibration signals acquired from experiments, including time domain, frequency domain, and time-frequency domain techniques.

3. Propose a reliable fault indicator for spalling development monitoring and develop a self-adaptive time-frequency technique for the extraction of critical information of fault diagnostic features.

4. In-depth investigation of the sources of various vibration signatures where unique wear patterns on the studied gears have been noticed and allocated to specific feature noted on the various vibration signals. The effect of the feature on spalling detection is also discussed. 


\subsection{Organization of the Thesis}

The thesis is constructed with seven chapters and is organized as follows: Chapter 1 provides a general introduction to machinery condition monitoring and common gear failure modes along with the contributions. Chapter 2 introduces sources and features of gear vibrations and reviews the various adopted signal processing techniques for gearbox fault diagnosis. In Chapter 3, an extensive description of the experimental setup and the configuration of gearbox test rig are provided. Spalling creation and details on the testing procedure are also described in this chapter. The mathematical equations and the algorithms in practical fault detection methods implementations are given in Chapter 4. This chapter also introduces the development of a self-adaptive time-frequency technique for gearbox fault detection. Chapter 5 provides in-depth descriptions of vibration features obtained from analytical results along with remarkable findings. A spall fault indicator with a capability to monitor the development of spalling is proposed in this chapter. In addition, this chapter explores the physical condition of the gear being studied and interprets the source of the unique vibration signatures of the gear. The last chapter concludes the findings and discoveries of this study along with possible future developments of this research. 


\section{Chapter 2}

\section{Literature Review}

This chapter gives an overview of general sources of gear vibrations and reviews some commonly-used diagnostic techniques related to gear defect.

\subsection{Vibrations from Gear}

Vibrations of gears are generated from the impact excitation forces during gear teeth meshing where two teeth are compressed and deformed under load. The deflection of teeth deviates from the ideal gear involute profile and introduces transmission error (TE) [4]. Other cause of TE may be on the account of gear teeth geometric errors and geometric modifications [5]. Geometric errors are imported during the manufacturing process unintentionally whereas geometric modification is introduced deliberately to reduce the impact of teeth mesh. A modified TE model was proposed in [6] to simulate the vibrations of a single stage gearbox in order to compute the TE. The model can be used to study the effect of tooth stiffness variation due to time-varying load points on the TE.

All vibrations from gears regardless their health state can be classified into two groups: mean effects for all tooth pairs and variations from the mean [7]. The first group is usually 
the results of (1) tooth deflection; (2) initial profile errors resulting from manufacturing tolerances and processes; and (3) uniform operational wear over all teeth. Teeth wear patterns usually appear on both side of the pitch line due to pure rolling action at the pitch line and sliding action on both sides. The effect of wear on the gear vibrations will usually be an increase in the second harmonic of gear mesh frequency (GMF), which provides useful information for monitoring development of teeth wear [4]. The second group can be caused by (1) random errors; (2) local faults; (3) slow variations; and (4) systematic errors. Slow variations can be non-uniform wear and will raise the energy of low harmonics of GMF. In contrast, both local faults and random errors could cause an increase in the magnitude of most harmonics of GMF and the associated sidebands. However, the magnitude of harmonics of GMF is primarily dependent on the transmission path between the source of vibration and the location where the sensor picks up the signal. Hence, different observations may be examined on the GMF harmonics of vibration data acquired from various locations and thus gear condition should be monitored based on the signals recorded from the same sensor.

Gear defects could generate modulation to the vibration of healthy gear and sidebands are formed around major sidebands of GMF harmonics. The associated sidebands of GMF harmonics, on the other hand, normally contain more diagnostic information [8]. Both local and distributed gear defects will cause amplitude and phase modulation, resulting in sideband formation or sideband variation in a spectrum [9] [10] [11]. The major cause of modulation effect is gear teeth stiffness variation due the presence of defects. Many researchers have been dedicated to studying the modulation effect on vibration signature from the dynamic perspective. Shengxiang and Ian created a 26 degree of freedom gear 
dynamic model to differentiate the effects of spalling and crack on spur gear vibrations. Their results suggest that the changes in amplitude modulation caused by spalling are negligible when compared to those caused by tooth root crack [12]. Fakher et al derived analytical expressions to study the change of gear mesh stiffness caused by spalling [13]. The analytical expressions were then applied to a one-stage spur gear dynamic model to quantify the reduction of gear mesh stiffness due to spalling. The authors indicated that gear mesh stiffness reduction introduces amplitude modulation in the way that it increases the number and the amplitudes of sidebands around the dominant GMF. A similar conclusion has been drawn from a theoretical calculation and experimental tests as outlined in [14] and [15].

\subsection{Gear Fault Detection Techniques Based on Vibration Signals}

Vibration-based analysis has been well established for gearbox condition monitoring in the past few decades. Feature extraction techniques are also extensively developed based on vibration signals. Those techniques can be broadly classified as time domain analysis, frequency/spectrum analysis and time-frequency analysis.

\subsubsection{Time Domain Analysis}

Time domain analysis is the simplest analysis method which features simple algorithms and requires the least processing efforts. The changes of the conditions of gearboxes may be indicated by various time statistical indices [16] [17]. Gear defects practically generate 
periodical impulsive excitation forces whose features can be picked up by an accelerometer. Statistical indices are then applied to the acquired signals to measure vibration energy produced from defects. They can be broadly divided into two categories: statistical measurement based on (1) raw signals and (2) preprocessed signals.

Root mean square (RMS), crest factor and kurtosis are widely adopted fault indicators, and they are commonly used on raw signals. RMS is effective to determine the overall peak level in gearbox condition monitoring, but it is unable to detect transient peaks in the signals generated by localized defects. On the contrary, crest factor and kurtosis measure the peakedness of the vibration signal, which estimates the transient peaks' energy due to gear defects [18]. As defects occur on gear teeth, impulsive excitation forces are generated and raise the vibration level which increases the number of peaks in the signal. Kurtosis characterizes the impulsive nature of the signal in fourth power. Similarly, the crest factor describes the impulsive nature in second power [19]. It is concluded from a study that kurtosis is a more sensitive indicator than the crest factor in measuring transient peaks of vibration signals because kurtosis requires less shock repetition period than crest factor [20]. The second category of statistical indices relies on preprocessed signals, which includes synchronous averaged signals, residual signals, and different signals.

Time synchronous averaging (TSA) is a signal processing technique which enables periodic features extraction from additive noise [21] [22]. For gearbox application, TSA separates the vibration signatures of the gear being monitoring from not only background noise but also the vibrations of the attached mechanical components whose rotation is nonsynchronized with the gear. The process of TSA requires the detailed information of the periodic frequency of the signals of the target shaft or a signal that is synchronized with 
the rotation of the target shaft. Digital resampling is employed in TSA to accommodate the effect of shaft speed variation and interpolation is proven to be an alternative to the phaselocked frequency multipliers [23]. McFadden [24] proposed a revised combo filter model for TSA, which is able to accurately extract the periodic waveform from noisy signals by applying a rectangular window to sample vibration signal in frequency domain. Enayet et al [25] performed TSA on the wavelet transformed signals across all the scales. Their results show that the processed signals effectively strengthen the periodic peaks generated by gear defects and the technique successfully detects both localized and distributed faults. Two secondary signals can be developed based on synchronous averaged signals, which are residual signal and difference signal [26]. Residual signal is obtained by removing shaft rotation frequency associated with its harmonics and the GMF along with its harmonics from SA signal, while difference signal is determined by eliminating the first order sidebands from the residual signal. The specific process of removing the desired frequency components can be accomplished with the implementation of ideal filtering which sets the spectrum target values to zero and inverse transforms the filtered frequency data to time domain. Several gear fault detection parameters are developed based on residual signal and difference signal, including FM4, NA4/NA4* and NB4/NB4* [19]. Choy et al [27] investigated the performance of those parameters on monitoring the development of gear pitting. They concluded that FM4 parameter is not sensitive to pitting development while NA4/NA4* is able to monitor the growth of gear damage and NB4/NB4* are similar to NA4/NA4* in addition to their ability to indicate the severity of pitting. However, those indicators are unreliable when the shaft speed variation is substantial since they are dramatically influenced by the shaft speed. Furthermore, they lack of the ability to 
distinguish local and distributed damage. Recently, a number of researchers have employed autoregressive modeling to extract residual signals from SA signals [28] [29] [30]. The techniques have been proven to be more effective and possess better performance compared to the traditional methods. Wang and Makis [28] used autoregressive residual signals along with K-S test which compares cumulative distribution functions of healthy and defective vibration signatures to detect the presence of gear shaft crack. The results, however, were obtained under the assumption of specific faults that are strong enough to alter the cumulative distribution functions of original vibration signature, for which the technique is not implementable for incipient gear defects such as pitting and spalling. Endo and Randall [29] proposed an enhanced autoregressive model to differentiate gear spalls and tooth fillet cracks. Minimum entropy deconvolution filter with an ability to characterize the phase information of gear vibration signal is embedded in the AR residual signal, combined with kurtosis to improve the performance of emerging gear tooth faults detection. Combet and Gelman [30] used spectral kurtosis (SK) as a filter to de-noise AR residual signals. The technique was applied to detect relatively small tooth surface pitting in a two-stage helical reduction gearbox and the results suggested that SK-based filtered residual signals gain higher signal-to-noise ratio and improve detection of initial nonstationary faults.

If defects develop on a gearbox, vibrations of the gearbox in healthy state are amplitude and phase modulated. The amplitude of overall vibration is modulated by a frequency component with lower order and the phase modulation usually happens on the dominant GMF. The fault features are hidden in the modulating components which can be revealed through the amplitude and phase demodulation. Narrow-band demodulation (NBD) is an 
early demodulation method which is achieved by band-pass filtering around the dominant GMF while the amplitude and phase information of the filtered signal can be obtained from analytic signal. McFadden [31] applied NBD to evaluate the amplitude and phase modulation due to gear fatigue crack in a gear tooth. He suggested that the location of the fatigue crack on the face width of the tooth may cause the change of phase angle of the time synchronous averaged vibration signals. Chen and Zuo [32], on the other hand, extracted characteristic frequencies generated by gear crack from the amplitude demodulated signals with adaptive spectrogram. The technique has been successfully implemented in both simulated and experimental vibration signals to detect gear cracks and the results were very prominent. For cases where the structural resonance excited by gear crack is located outside the dominant GMFs, NBD is less effective in extracting vibration signatures of gear faults. Wang [33] proposed an application of resonance demodulation on a particular case. Resonance demodulation is performed on a band-pass filtered residual signal instead of a solely synchronous averaged signal. Then structural resonances excited by gear tooth crack can be detected within the range of gear meshing harmonics. The author has demonstrated the remarkable implementation of resonance demodulation in particular situations with experimental data.

\subsubsection{Frequency Analysis}

Gearbox components are vibrating at different frequencies and these frequencies contain unique vibration signatures of individual component and vary along with the change of applied load and driving speed. Monitoring the variation of particular frequency bands 
provides evaluation of the condition of the corresponding vibrating components. Faults can be detected by comparing the spectrum of a damaged gearbox with the spectrum of a healthy one [34]. After gear vibration signals are preprocessed with TSA, the signals contain only the vibration signature of the gear of interested in exact one revolution. During TSA, due to the application of digital resampling to accommodate the shaft speed fluctuation, the equivalent sampling frequency are changing continually. In order to interpret the frequency components on a spectrum, the frequency axis is expressed in terms of the rotational rate of the shaft of interest with the unit order. Local defects such as surface pits and fatigue cracks generate impacts which excite resonances of the gearbox over a wide frequency range [35]. Low-pass-filter can be applied to isolate the strong resonance in low-frequency range and the filtered signal provides diagnostic information. As mentioned earlier, sidebands around the GMF harmonics are results of modulation due to gear faults, so faults can be identified by monitoring the behavior of sidebands. El-Shafei's [36] experiments showed that significant sidebands occurred on enveloped signals obtained from a gear with excessive wear whereas the sidebands disappeared after the faulty gear was replaced. Dalpiaz et al [37] examined the activities of sidebands due to gear cracks and they concluded that local cracks mainly modify the sidebands around dominant harmonics of GMF. Diwakar et al [38] conducted an experiment to simulate a tooth-broken gearbox failure by removing two teeth from a gear artificially. The spectrum of defective gear clearly indicated the presence of peaks at sub-harmonics of GMF. Gear defect can be also detected using the envelope spectra of high frequency random signal [39]. The technique is capable of identifying gear defects at the earliest stages of development, even when the machines are operated at low speed, in which the gear defects are non-detectable 
with narrow band pass signals.

Cepstrum was first proposed by Bogert [40] to detect echoes in seismic signals. It is defined as "the power spectrum of the logarithm of the power spectrum". The application of cepstrum is further extended to mechanical problems, such as gear fault diagnosis [41]. Spectrum is the measure of the periodicity of time signal while cepstrum highlights the periodicity of spectral components. Cepstrum, therefore, can be used to evaluate abnormal activities of GMF and its harmonics as well as the associated sidebands. It can be concluded from the above reviews that sidebands provide significant diagnostic information. However, in the case of an incipient defect, the global vibration signatures are not significantly affected to alter the number and amplitude of the main sidebands [37]. On the other hand, the defect may vary the spacing of the sidebands and it can be detected by cepstrum analysis [42]. Order cepstrum analysis has been proven to be successful in identifying a small pit in a vehicle gearbox as outlined by Morsy and Achtenová [43]. The authors have demonstrated that order cepstrum is a sensitive fault indicator for early gear damage. Badaoui et al. [44] have developed a robust defect identifier called moving cepstrum integral $(\mathrm{MCI})$ which utilizes the merit of power cepstrum that enables the isolation of defect impulses and no-defect excitations. MCI was employed in both numerical and experimental data and the results were consistent. The authors indicated that MCI is capable of spalling detection and localization. Further research has confirmed the robustness of power cepstrum as a gear defect indicator [45]. Frequency analysis has been proven to be popular in machinery condition monitoring due to its strong mathematical basis and ease of application. However, the property of frequency transformation limits its ability to handle non-stationary signals. Fourier transform measures the energy of existing 
frequency components of a signal across the entire time, which is applicable when the analyzing signal is stationary. Gearbox signals are usually non-stationary and their frequencies vary along with time, hence spectrum/cepstrum cannot provide comprehensive analysis on the condition of gearboxes.

\subsubsection{Time-Frequency Analysis}

Time-frequency analysis is developed according to the need for non-stationary signal analysis. The earliest time-frequency method is the Short Time Fourier Transform (STFT) which was introduced by Gador [46] to analyze speech signals. STFT assumes that a nonstationary signal is locally stationary in a desired time period and the corresponding frequency components are determined by Fourier transform. This is accomplished by applying a window function with a certain size that moves along time axis to perform localized FT [47]. Safizadeh et al [48] applied STFT to diagnose a gearbox with a broken tooth. The time-frequency representation of STFT clearly shows that an abnormal pulse occurs near the GMF of $400 \mathrm{~Hz}$ and repeats every $0.66 \mathrm{sec}$. They concluded that the abnormal pulse is due to a gear broken tooth and they were able to determine the damaged gear according to the repetitive frequency of the pulse. Mosher et al [49] developed a new metric for gear damage detection that employed STFT as one of the time-frequency transforms. The STFT embedded metric produced systematic numerical representation which indicates the changes in vibration signatures of a damaged spiral bevel gear. Polikar [50] illustrated an example to demonstrate the effectiveness of STFT in analyzing nonstationary signals. He created two signals in which one is stationary and the other one is 
non-stationary. The spectrums of the two signals are identical whereas the time-frequency plot of SFTF is able to distinguish the two signals. He also pointed out that the disadvantage of STFT is the time-frequency resolution problem. STFT can provide good resolution in either time domain or frequency domain but not both due to Heisenberg Uncertainty Principle. In some cases, signal analysis prefers high frequency resolution at low frequency components and fine time resolution at high frequency transients. STFT with fixed windows cannot achieve this desired analysis.

The concept of wavelet was frequently discussed in the early 1980s, which is the synthesis of ideas from mathematics, physics and engineering. Jean Morlet [51] applied the concept of wavelet and developed the Continuous Wavelet Transform (CWT) to provide a solution for the time-frequency resolution problem associated with STFT. He proposed a basis function called Mother wavelet to form a wavelet family by translation and dilation of mother wavelet. The wavelets are used to decompose a signal by convolution. The dilated wavelets obtain a feature of narrow bandwidth in frequency domain so they preferably pick up low frequency components with fine frequency resolution; while the compressed wavelets with short time duration have analogous signatures as the high frequency transients, which transient structures of a signal are efficiently detected with a good time resolution. Due to the effectiveness of wavelet in analyzing non-stationary signals, it has been widely applied to gearbox condition monitoring where it is progressively developed for detection of various gear damages. Staszewski and Tomlinson [52] used wavelet transform to create contour plots for signals for a spur gear with a broken tooth. Then they calculated the statistical similarity measurements between the healthy and the damaged signals, called the Mahalobis distance. Their results suggested that the Mahalobis is a more 
sensitive fault indicator than the kurtosis of the envelope signals. Wang and McFadden [53] attempted to detect a fatigue crack in a helicopter gearbox with orthogonal wavelets such as Daubechies 4 and 20. However, they concluded that although orthogonal wavelet transform provides fast algorithms and no redundant information, the wavelet amplitude map lacks the ability to describe small variations from gear defect due to the limited number of scales of orthogonal wavelet transform. Their subsequent research [54] employed non-orthogonal wavelets such as Morlet wavelet to differentiate the vibration signatures generated by localized and distributed gear defects. Morlet wavelet is the most popular mother wavelet for gearbox fault detection since the nature of Morlet wavelet is highly similar to the impulse vibration generated by gear defects [52]. Further studies have shown that CWT is a prominent and powerful tool for gearbox condition monitoring [55] [56] [57] [58] [59] [60].

Discrete wavelet transform (DWT) is a derivation of CWT where DWT discretizes the scale and translation parameters of wavelet function to form wavelets and scaling functions [61]. Then any signal can be decomposed in terms of wavelets and scaling functions. Dyadic discretization is the most popular wavelet discretization that provides a fast computerized implementation. Discretization process can be achieved by recursively lowpass filtering and down sampling the signal [62]. Signal reconstruction is the reverse process of the decomposition that involves up sampling and high-pass filtering. DWT thereby has advantages of less computational effort and no redundancy over the CWT. It has been effectively applied to extract features excited by gear faults. The feaures were then fitted into a neural network algorithm able to recognize the changes caused by gear 
faults as well as classify various gear damage modes [63] [64]. The problems introduced by DWT, however, limited the spread of its application and development of more advanced techniques became necessary. As pointed out by Selesnick [65], DWT causes four main problems: coefficient oscillations, shift variance, aliasing and lack of directionality. Kingsbury [66] proposed a relatively recent technique that provides an alternative to DWT, called dual-tree complex wavelet transform (DTCWT), which has properties of nearly shift-invariant and directionally selective and perfect reconstruction. DTCWT applies two different sets of low-pass and high-pass FIR filters to perform two parallel DWTs. Two sets of wavelets and scaling coefficients are assembled to form an analytical wavelet. DTCWT was originally designed for image processing applications but was further implemented for gear crack detection [67]. Results obtained from DTCWT were compared to results from second-generation wavelet transform and the demonstration proved that DTCWT offers better shift invariance and less spectral aliasing. The frequency response of DTCWT [66] indicated that the technique does not completely remove spectral aliasing since the filters cross over to the negative frequency plane at both low and high-frequency bands. The cause is due to the fact that the FIR filters are finite support and the resulting wavelets are approximately analytic instead of fully analytic [65].

EMD is an elaborately developed method for analyzing non-stationary data proposed by Huang [68]. The idea of EMD is the decomposition of non-stationary signals into a finite number of intrinsic mode functions (IMF) that allow accurately calculation of instantaneous frequencies. EMD is an adaptive signal decomposition technique since it is based on the local characteristic of a signal. Therefore, EMD has the advantage over the non-adaptive wavelet coefficients whose correlation with a signal is restricted by the nature 
of mother wavelet. Researchers have adopted the principle of EMD and have widely applied it to machinery fault diagnosis. Loutridis [69] calculated the energy of the second IMF and used it as a fault indicator to detect a gear crack from vibration signal of a dynamic model and a test rig. Wang [70] extended the method to derive a prediction curve based on average energy values for the prediction of tooth crack development. The outcome showed that the predicted curve is consistent with experimental results, and the author proposed that EMD scheme can be used to monitor the condition of gearbox systems. Cheng [71] applied energy operator to extract instantaneous amplitude and phase from IMFs. The energy operator reduces window effect due to Hilbert transform and provides more precise signal demodulation. However, it is indicated that energy operator can be applicable for only mono-component signals, i.e. IMFs. The performance of energy operator-based demodulated signals is evaluated through implementation of the method for detecting a fault on the outer race of a roller bearing. The results show that energy operator based EMD is a more reliable diagnostic technique than the conventional Hilbert transform based EMD. Teng [72] et al compared the effectiveness of fault modulation information extraction between Hilbert transform based demodulation and EMD on the data collected from a wind turbine gearbox. The results show that EMD is more superior in detecting incipient gear defects such as pitting than Hilbert transform based demodulation. The main drawback of EMD is mode mixing problem in which disparate frequencies occur in a single IMF [73]. Mode mixing is usually caused by signal intermittency in which the physical meaning of individual IMF may be interpreted differently. Another implication of mode mixing is that energy leaks from one IMF into adjacent IMF. As a consequence, the decomposed signals are inadequate due to lack of inherent orthogonality characteristic of IMFs caused by 
energy leakage [68]. Ensemble empirical mode decomposition (EEMD) is proposed to attenuate the effect of mode mixing with additional white noise to the original signal [73]. Research experiments have demonstrated that EEMD is a powerful tool and offers prominent application in gearbox fault diagnosis [74] [75] [76].

In this thesis, TSA is primarily used as a preprocessing tool and the processed signals contain only the vibration signatures of the gear of interest. All the techniques mentioned in this thesis are applied to the TSA signals. Then the signals are transformed into frequency domain for spectrum analysis. Amplitude and phase demodulation are employed to extract the modulating components. Continuous wavelet transform is used to obtain the time-frequency analysis. For more advanced vibration signatures analysis, EMD is implemented to decompose the TSA signals of several IMFs which contain important features of a gear defect. 


\section{Chapter 3}

\section{Experimental Setup and Testing Procedure}

The thesis has been forcing on the development of diagnostic techniques towards gear spalling. A spall was manually introduced to a pinion of an accessory gearbox of an aircraft turbojet engine. Prior to this study, a test rig was constructed based on a real operating system of the gearbox at the National Research Council Canada. The testing procedure was deliberately designed to conduct experiments that provide representative data. This chapter gives extensive descriptions of the test rig, spalling creation and detailed testing procedure.

\subsection{Testing Gearbox}

The test gearbox is a real accessory gearbox of a J85 engine which is equipped on several aircrafts such as Canadair CT-114 Tutor [77]. The gearbox is integrated at the bottom of the engine, as shown in Figure 3-1. It is mainly composed of four parallel shaftgears supported on rolling-element bearings at their extreme ends (Figure 3-2). For illustration convenience, each shaftgear was allocated a letter. Spur gears are solely used in the gearbox to reach high power transmission efficiency and obtain ease of installation. 
The accessory gearbox has several functions on an aircraft such as: 1) transmits power from the engine to the fuel pump; 2) powers electric equipment of the aircraft, 3) drives the various hydraulic systems, 4) start the engine and, 5) supplies lubricant to the various engine and gearbox bearings. Figure 3-3 depicts the gearbox and its accessories. Shaftgear $\mathrm{B}$ is the main drive shaft which is driven by the engine through a transfer gearbox. The other end of shaftgear B is coupled to an overspeed governor, limiting the rotation speed of shaftgear B below $7800 \mathrm{rpm}$. During an aircraft take-off, shaftgear B reaches $80 \%$ of its maximum speed, accounting for $6240 \mathrm{rpm}$. While the aircraft is in cruise state, the rotation speed of shaftgear B is maintained at about $3666 \mathrm{rpm}$, which represents $47 \%$ of the maximum speed. Shaftgear D is directly connected to a fuel pump, and it drives an oil pump via an internal gear ring (Gear OP). Shaftgear $\mathrm{F}$ is a pinion which contains two identical gears integrated on one shaft. Power is transmitted to a hydraulic pump and a starter/generator through shaftgear C.

Table 3-1 gives the number of teeth for each gear while the rotation frequencies of all shafts are given, under three operating conditions, in Table 3-2. The test gearbox was acquired by NRC from Orenda's overhaul centre after being in service for an unknown period of time. In addition to its previous operation on an aircraft, the gearbox was previously tested with a root-cracked tooth for more than one hundred hours [78]. Its condition at the beginning of this study had a significant impact on the results that will be discussed later in the thesis. 


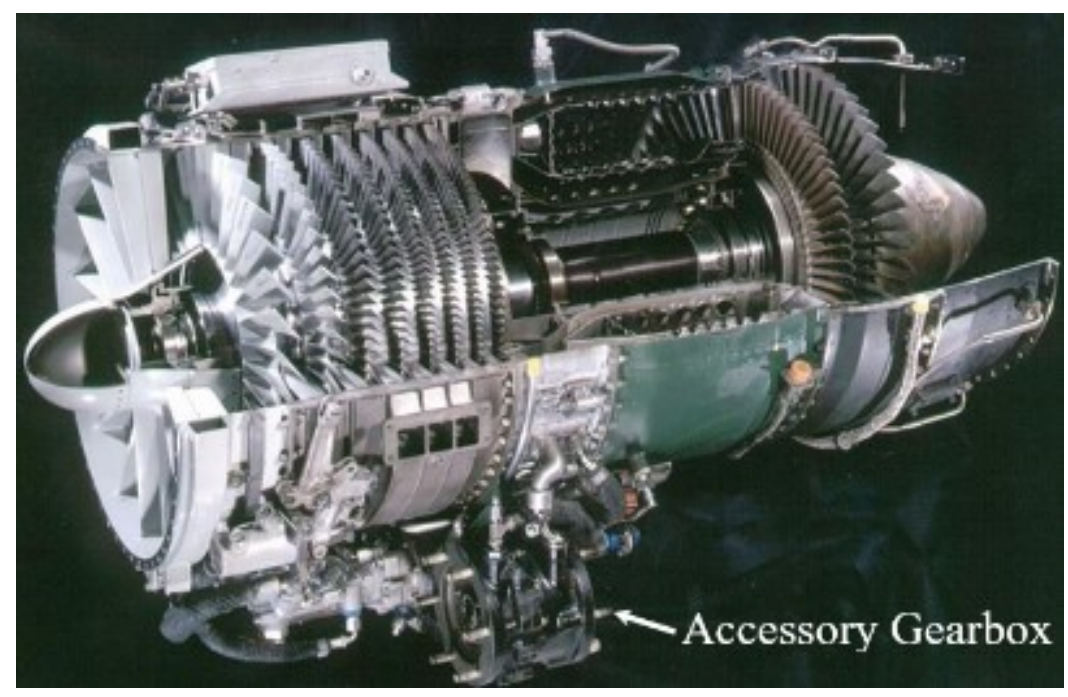

Figure 3-1 General Electric J85 engine and its accessory gearbox [79]

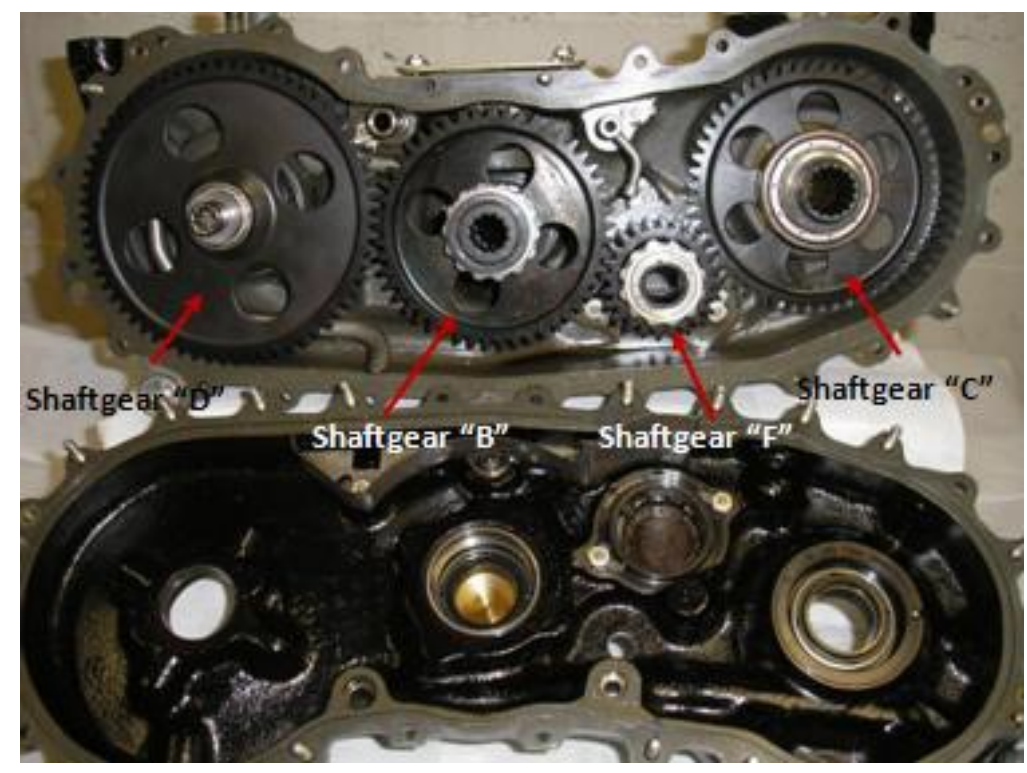

Figure 3-2 Accessory gearbox configuration [78] 


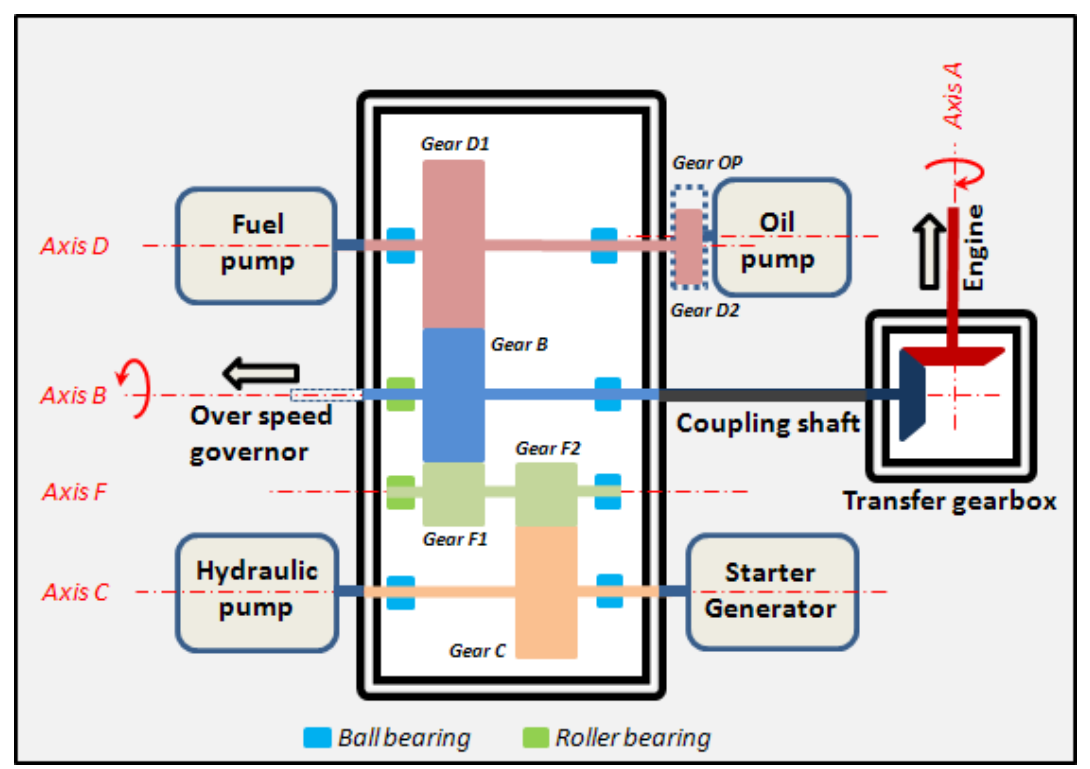

Figure 3-3 Schematic of the accessory gearbox and attached mechanical accessories [78]

Table 3-1 Gear specification

\begin{tabular}{|l|c|c|c|c|c|c|c|}
\hline & \multicolumn{7}{|c|}{ Shaft } \\
\cline { 2 - 8 } & Shaftgear B & Shaftgear C & \multicolumn{2}{|c|}{ Shaftgear D } & \multicolumn{2}{|c|}{ Shaftgear F } & $\begin{array}{l}\text { Oil } \\
\text { Pump }\end{array}$ \\
\hline Gear & B & C & D1 & D2 & F1 & F2 & OP \\
\hline $\begin{array}{l}\text { Number } \\
\text { of teeth }\end{array}$ & 49 & 54 & 63 & 20 & 24 & 24 & 29 \\
\hline
\end{tabular}

Table 3-2 Rotation frequencies of shafts and gear meshing frequency

\begin{tabular}{|l|c|c|c|c|c|c|}
\hline \multirow{2}{*}{ Speed } & shaft B & shaft D & shaft C & shaft F & Gear mesh & Oil pump mesh \\
\cline { 2 - 7 } & $\mathrm{Hz}$ & $\mathrm{Hz}$ & $\mathrm{Hz}$ & $\mathrm{Hz}$ & $\mathrm{Hz}$ & $\mathrm{Hz}$ \\
\hline Maximum & 130 & 118 & 101 & 266 & 6379 & 2025 \\
\hline Take-off & 104 & 95 & 81 & 213 & 5103 & 1620 \\
\hline Cruise & 61 & 56 & 48 & 125 & 2998 & 952 \\
\hline
\end{tabular}




\subsection{Test Rig Description}

As described in the previous section, the test facility used to conduct the experiments is NRC's gearbox test rig (Figure 3-4). In order to simulate real operating environment of the gearbox, a number of changes have been brought to the various accessories as will be described below. The test rig is assembled on a steel frame which is supported by a second steel frame meanwhile the second frame is secured to the ground with bolts. Contact between upper and lower frames is padded with rubber sheets to isolate vibration transmission from one to another. The gearbox is solidly bolted to the top bar of the upper frame, enclosed by various mechanical components. Since it is economically impractical to implement a turbojet engine as the power source in laboratory, a $15 \mathrm{~kW}$ variable speed electric motor is used to drive the gearbox via a pulley-belt mechanism connected to shaftgear B. The parameters of the pulley-belt system are deliberately designed to prevent the main drive shaft of the gearbox from exceeding the limited speed when the electric motor outputs its maximum speed. The electric motor is equipped with a variable frequency drive which is a remote speed controller that enables the gearbox reaching desired speeds at a constant rate. 


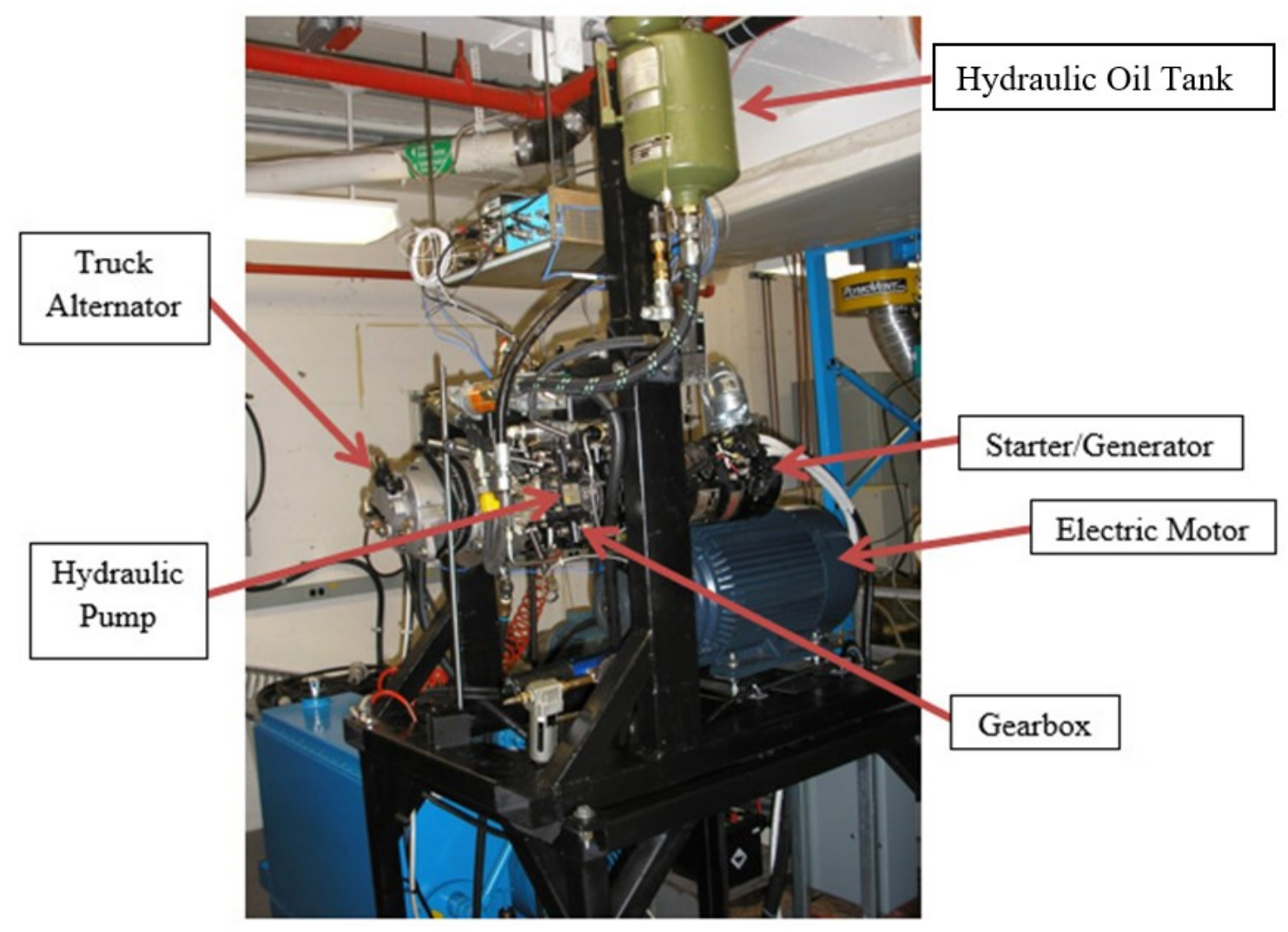

Figure 3-4 Overview of the test rig 
A major modification of the test rig assembly from original engine system is the lubrication system. The oil pump installed on the right side of shaft D (Figure 3-3) circulates oil to all components requiring lubrication in the engine/gearbox assembly (bearings and gears). The pump consists of one pressure and five scavenge elements, as indicated in Figure 35a. The pressure element pumps oil from the oil tank (integral to the pump) into the accessory gearbox then redirects the flow to the transfer gearbox and bearings and power take-off assembly of the engine, through a system consisted of internal channels, flexible pipes and nozzles. Oil and air mixture forms during the lubrication process and are drawn back to the oil tank by the five scavenge elements. As the engine and transfer gearbox are replaced by an electric motor and pulley-belt mechanism respectively, modifications to the lubrication system have been made to accommodate the changes in order to simulate proper operating conditions of the oil pump. Two orifice restrictors are used to replace the engine and transfer gearbox elements in the lubrication system, in which pressure flow diverges into scavenged flows. Due to the formation of oil and air mixture within the engine, one of the orifice restrictors has one mouth connected to an opened check valve on a vent tube. A detailed configuration of the modified lubrication system is shown in Figure 3-5b.

The fuel pump (Figure 3-3) on the left side of shaft D is replaced by a truck alternator (Figure 3-6) to simulate the power drawn by the fuel pump. It is powered by a $12 \mathrm{~V}$ battery and the amount of voltage delivered to the truck alternator is adjusted by a load bank which is integrated with five amperage switches. The hydraulic pump on the left side and the starter/generator on the right side of shaft $\mathrm{C}$ also consume significant parts of the engine power. For testing purposes, the hydraulic pump is set to simply circulating oil between itself and the hydraulic oil tank. A pressure regulator is installed in between hydraulic pump 
and oil tank to maintain the hydraulic pressure in the range of $9.65 \mathrm{MPa}$ to $11.4 \mathrm{MPa}$ at different input speeds as instructed in the operation manual of the gearbox. The starter/generator, as indicated by literal meaning, starts the engine and generates power for electric apparatuses on an aircraft. During the experiments, only the generator is used and its output voltage is regulated around $28 \mathrm{~V}$ by a voltage regulator. The generator is loaded by a multiple-switch load bank. During the experiments, three electrical loads (100 A, 140 A and 167 A) are applied to the gearbox through the load bank. The load settings are achieved by estimating the total electrical power consumption of a typical aircraft (Falcon 20) under three operational scenarios. Falcon 20 is propelled by two CF700 engines which are the improved version of the J85 engine with similar accessory gearboxes. 


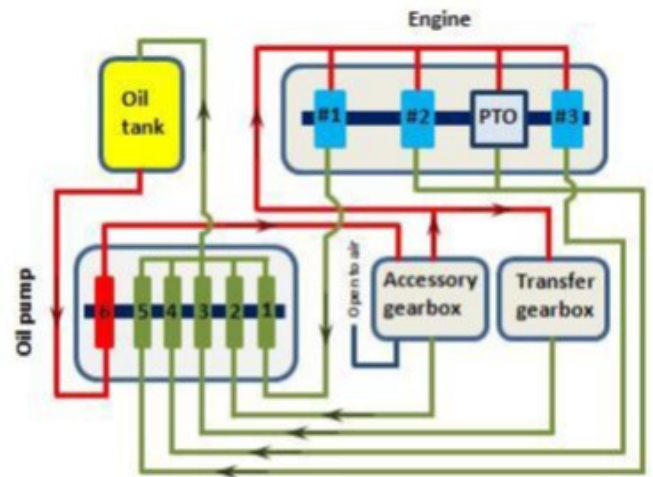

a.

Bearing $\square$ Powertake-off assembly

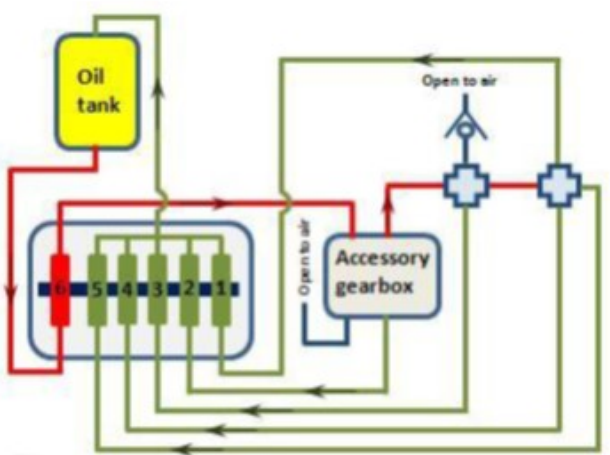

b.

Figure 3-5 Schematic of lubrication system: a. original, b. modified [78]

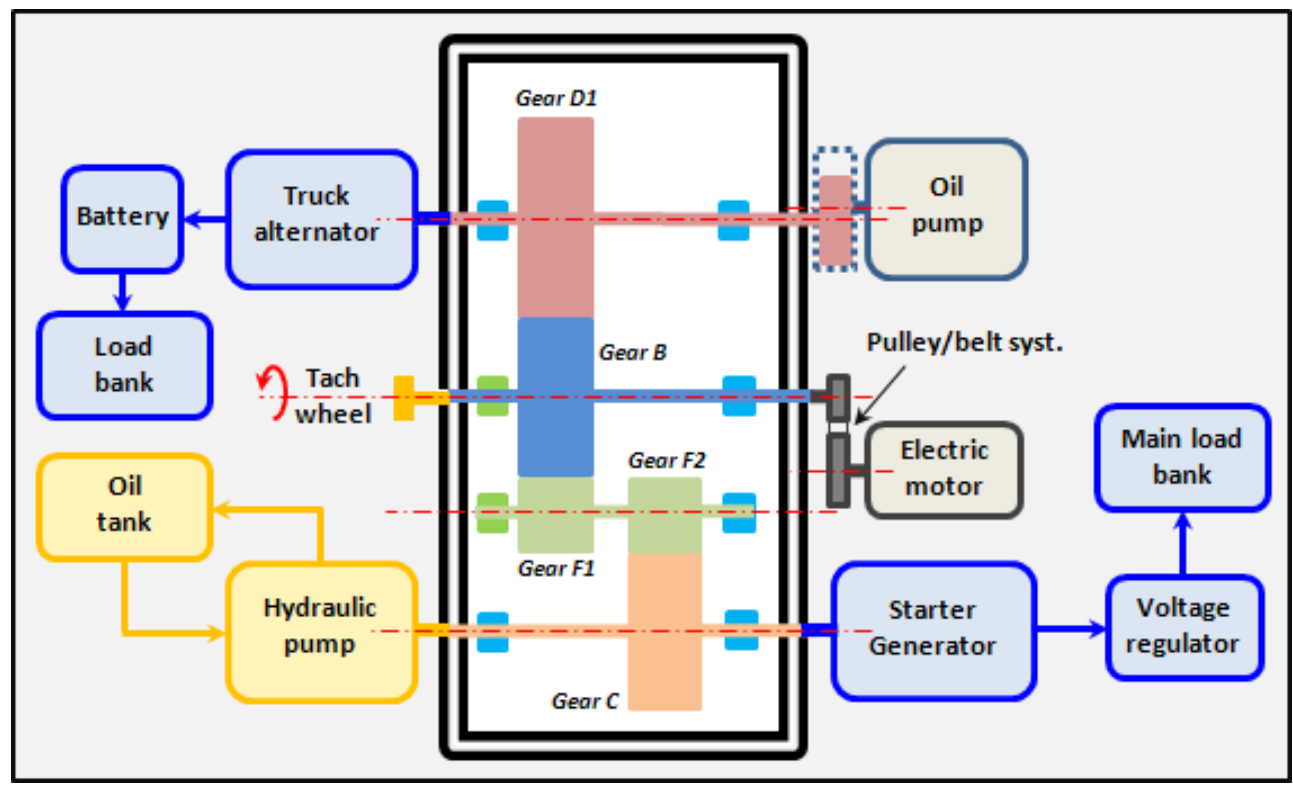

Figure 3-6 Schematic of gearbox test rig [78] 


\subsection{Instrumentation}

The test facility was instrumented by various sensors providing the measurements of dynamic and static signals of different parameters characterizing the rig in operation. Dynamic signals relate to the condition of the accessory gearbox which includes vibration and acoustic emission signals, whereas static states refer to the operating environment of the gearbox, i.e., temperature, pressure, power consumption, speed and applied load. In total, three accelerometers and two acoustic sensors are used to measure dynamic signals. As shown in Figure 3-7, vertical front (VF), vertical back (VB) and horizontal (HR) accelerometers were stud mounted in three different locations of the gearbox housing to measure the vibration signals. All sensors including both dynamic and static measurements are wired to a LMS data acquisition system (Figure 3-8) which was set to acquire the signals at a sampling rate of $25,600 \mathrm{~Hz}$. The captured data were saved in a computer connected to the LMS system. Since static signals do not utilize the maximum capacity of the system, they were sampled at a much lower rate.

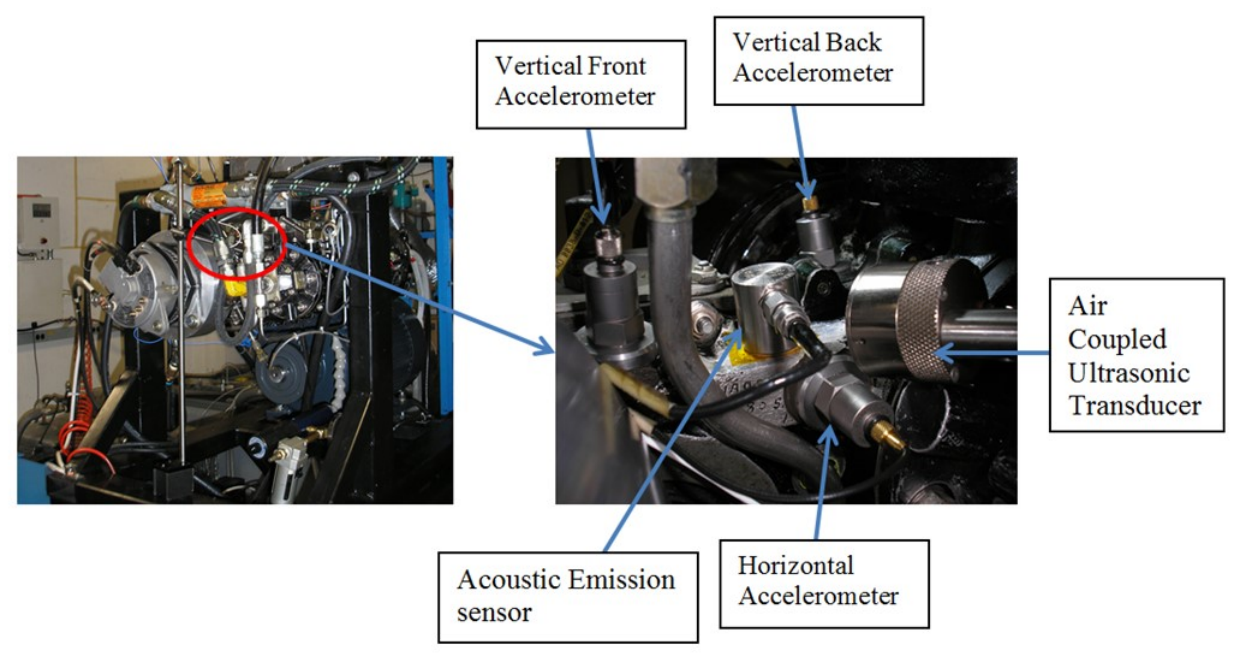

Figure 3-7 Gearbox condition monitoring sensors 
Air-coupled ultrasonic transducer (ACUT) outputs voltage proportional to pressure wave arriving at its aperture. The frequency response of ACUT reaches the maximum of 2.25 $\mathrm{MHz}$ with a $6 \mathrm{~dB}$ down point at the upper frequency. More detailed information on the ACUT can be found in the literature [80] [81]. ACUT is placed towards the upper edge of the gearbox housing in order to reduce the effect due to signal weakened by the distance between the source to the transducer. Acoustic Emission (AE) sensor converts the transient elastic waves generated by the release of strain energy to an electrical signal [82]. AE sensor has a resonant frequency at $29 \mathrm{kHz}$. At the peak sensitivity, it is able to operate from 25 to $70 \mathrm{kHz}$. The AE sensor is glued on the top of the gearbox housing. Due to calibration issues during the experiments, signals from the AE sensor were distorted and consequently have not been considered in this analysis. A tachometer measures the speed of shaftgear B through a thirty-tooth wheel coupled to the end of shaftgear B as shown in Figure 3-9. The tachometer is used to measure the speed of shaftgear B and outputs a sequential square wave with a maximum of $5 \mathrm{~V}$ and minimum of $0 \mathrm{~V}$. The speed of shaftgear $\mathrm{B}$ can be determined by calculating the period of 30 pulses. Another significant application of tachometer signals is to provide the phase information of the gear of interest for the determination of SA signals. 


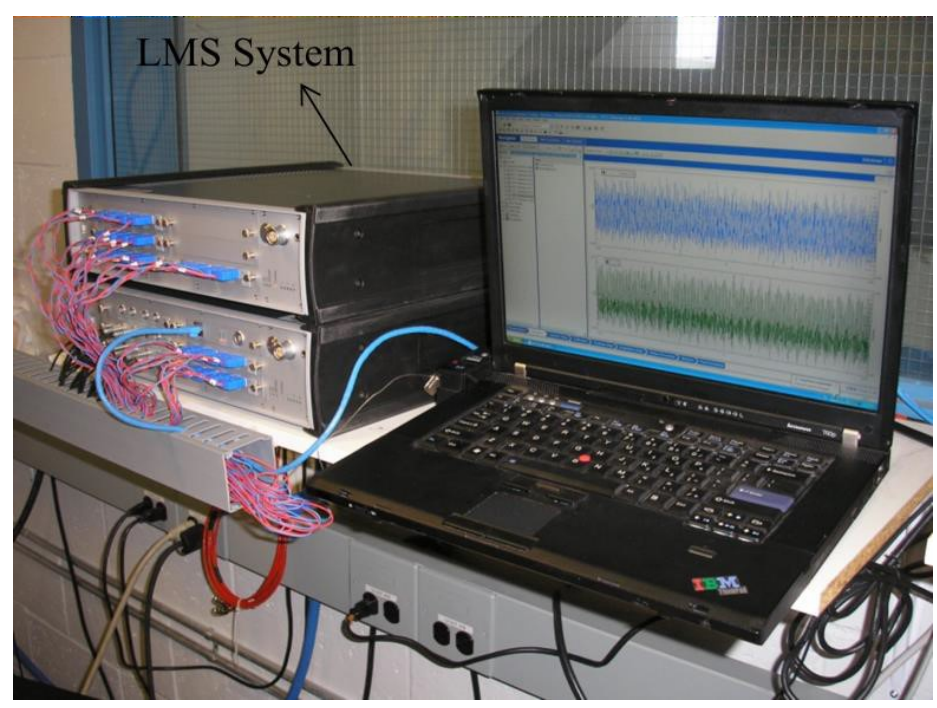

Figure 3-8 Data acquisition system

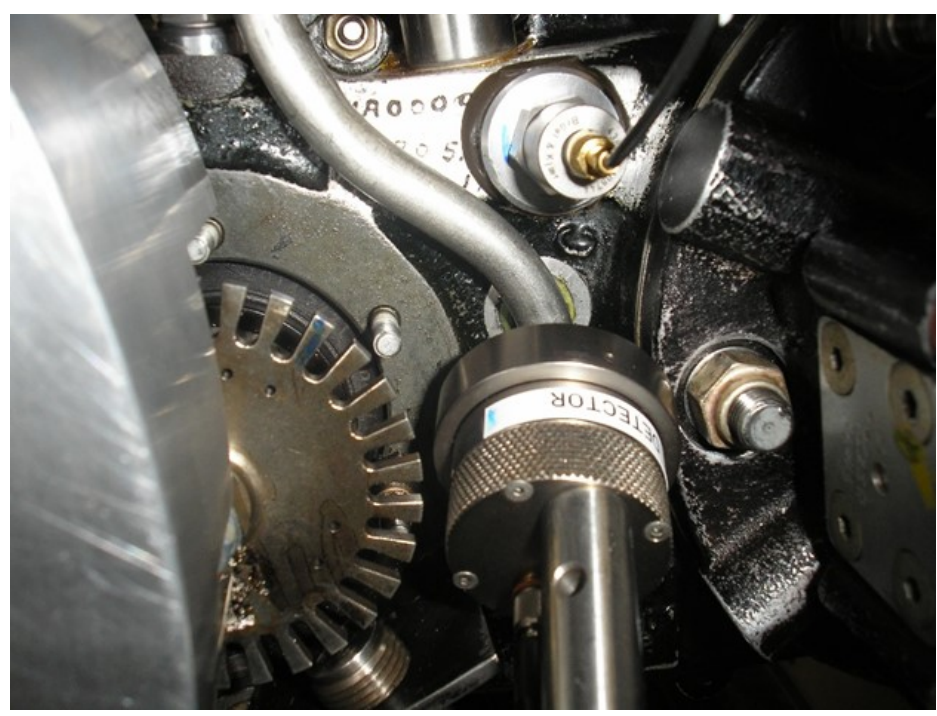

Figure 3-9 Tachometer coupled to the gearbox 


\subsection{Defect Creation}

The goal of the research project is to identify spalling on gear tooth which naturally occurs due to the high compressive contact stress. Such defects may occur after significant operation/testing time which is impractical within a laboratory environment. Considering its high expense in both cost and time, it is usually impractical to run the gearbox until a spall develops on the gear. As a follow-up study to a previous study focused on tooth cracking faults, a spall been then manually introduced to one of the gear teeth. For this purpose, gear F2 was chosen as the studied defected gear. As mentioned in Chapter 2, a spall appears as an irregular cavity at a tooth surface near the contact pitch circle of a depth

of 20-100 $\mu \mathrm{m}$. A spall of two different sizes with similar shape are created using a rotary power tool. The location of the spall was chosen to be particularly on the pitch line where the highest pressure is experienced during teeth meshing. The first spall, denoted D1 (Figure 3-10), covers one-ninth of the total tooth surface (meshing side). The depth and width of the spall are approximately $1.3 \mathrm{~mm}$ and $3.5 \mathrm{~mm}$ respectively. The second spall (D2) was enlarged based on D1 where the defected area was extended to one edge of the tooth surface as shown in Figure 3-11. D2 simulates a growth of defect D1 as a result of high stress taken by a smaller contact area. 

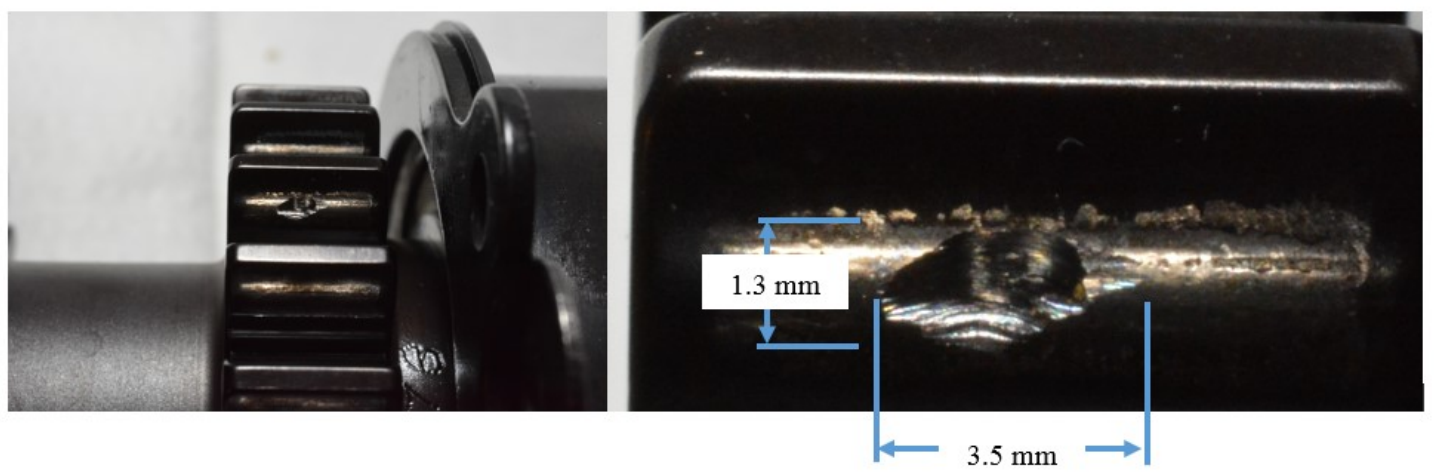

Figure 3-10 D1 Spall
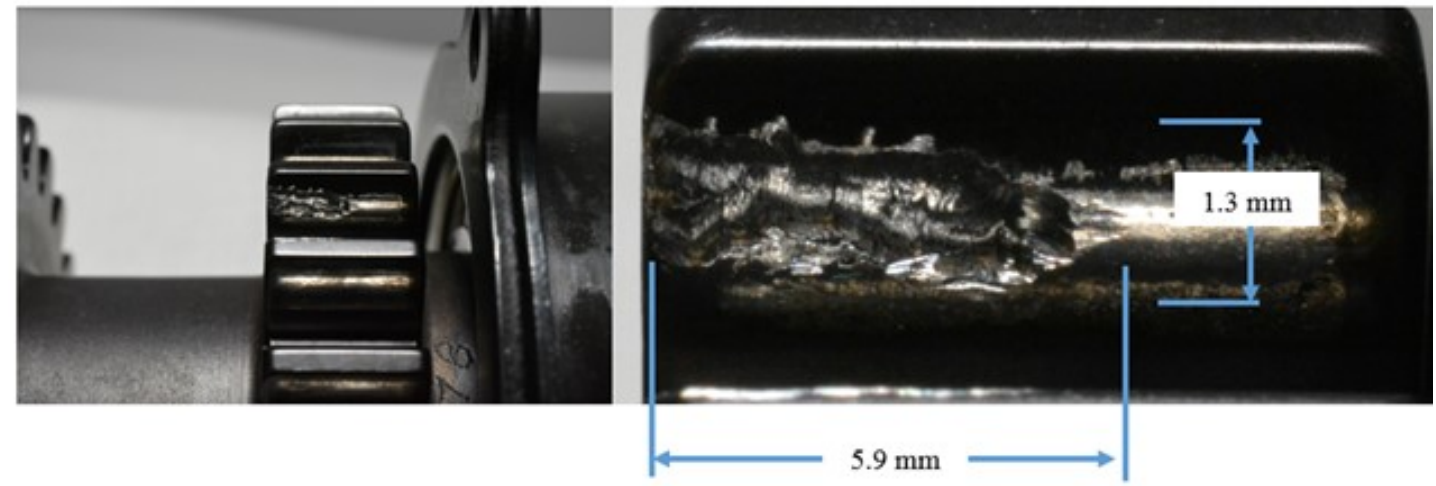

Figure 3-11 D2 Spall 


\subsection{Testing Procedure}

Three experiments were conducted following a similar procedure. To better describe the testing procedure, these three experiments are called baseline test, D1 test and D2 test, respectively, in the next chapters.

A baseline test is conducted on a non-defected gear. However, the gearbox has already served in the field and incurred normal operational wear on the various gears especially at the teeth meshing surfaces. Testing procedure steps are listed as follows:

1. Gearbox ramp up process. The speed of shaftgear B is gradually increased to $3671 \mathrm{rpm}$ which is the estimated speed of shaft B when the aircraft is in cruise.

2. Load condition 1. The alternator was loaded to 7A; meanwhile, the generator was loaded to $112 \mathrm{~A}$. Six recordings were collected and each recording spans for 32 seconds. For the next two load conditions (on the generator), the load applied on the alternator remains constant.

3. Load condition 2. Generator is loaded to $138 \mathrm{~A}$. Six recordings were collected.

4. Load condition 3. Generator is loaded to 167 A. 6 recordings were collected.

5. Speed ramp down process. The speed of shaft B ramp down from cruise speed to zero and the process was recorded.

Both D1 test and D2 test followed the same procedure. D1 test was conducted on the pinion gear that contains a D1 spall while D2 test was conducted on the pinion gear with a D2 spall. 18 recordings were collected for both tests respectively. 


\section{Chapter 4}

\section{Signal Processing Techniques}

This chapter provides detailed description of signal processing techniques used to analyze the acquired data. The description includes the mathematical equations and the algorithms in practical implementations. All collected data are outputted into MatLab files to be processed using the various signal processing functions. Total 54 MatLab files were generated and classified under 3 groups of data representing the signals of the three health conditions of the gear under study. Each file contains both dynamic and static measurements, however only dynamic signals are analyzed and presented in this study. The amount of information corresponding to the condition of the gearbox is significantly sensitive to the transmission path from the source of vibration to the location of a sensor. Both VF and VB measure vertical vibrations of the gearbox. VF is closer to the source of damage compared to the VB sensor and consequently the VF signals contain more features and provide consistent data based on analysis. HR sensor measures the vibration of the gearbox in the horizontal direction and based on data analysis, its signals contain very little information to be related to gear fault detection. Therefore, only signals acquired using the VF sensor are processed, analyzed and discussed in this thesis with representative results shown in this chapter. 


\subsection{Time Synchronous Averaging (TSA)}

TSA triggers the collected raw data with respect to a tachometer pulses and then averages the data based on a single revolution of the gear of interest. The mathematical basis of TSA is given by the following equation [83]:

$$
y(n)=\frac{1}{N} \sum_{r=0}^{N-1} x(n+r M), \quad n=0, \ldots, M-1
$$

where $x$ is a sequence of vibration signal; $N$ is the number of averages; $M$ is the estimated period in one revolution, with the unit of the number of data per revolution.

Basic TSA algorithm starts by dividing a vibration signal of the gear of interest into segments that each segment contains the vibration signatures of the gear in exact one revolution. Then TSA is obtained by averaging all the segments. In this study, TSA is implemented as a preprocessing step based on the algorithm provided by Bechhoefer and Kingsley [84]. Once TSA signals are obtained, further signal processing is implemented for fault diagnosis purposes.

The data logging system possesses a sampling frequency of $25,600 \mathrm{~Hz}$ with a time length of 32 seconds for each recording; therefore, one recording contains 819200 data points. Based on the TSA algorithm, the estimated average number of data points per rotation of gear F2 is 208, which gives approximately 3938 averages per recording during the TSA process. As the vibration signals of the target gear in one revolution are averaged over 3900 times, any vibration that is not synchronized with the rotation of the target gear tends to be 
eliminated, maintaining only the vibration of the target gear in one revolution. Figure 4-1 is plotted using the data from a baseline test signal. It shows that the TSA signal has much lower amplitude than the raw signal, and quantitatively the RMS of the raw signal is 22.3 $\mathrm{g}$ while the RMS of the TSA signal is reduced to $16.8 \mathrm{~g}$. The decrease in RMS of vibration signal after synchronous averaged is mainly due to vibrations removal of asynchronous components.

Figure 4-2 shows synchronous averaged signals of baseline, D1 and D2 tests respectively. It is observed that the gear F2 is vibrating at an amplitude between $-40 \mathrm{~g}$ and $40 \mathrm{~g}$ for all gear conditions when the gearbox is loaded with load condition \#3. The vibration features of the baseline TSA signal can be generally described as a repeating pattern which is constructed by two relative large jumps connected by three small jumps. Under the effect of spalling, the characteristic of these patterns is altered so that the amplitude of those three small jumps in baseline TSA signal are increased, resulting in the increase of the overall vibration amplitude. On the other hand, the RMS of the baseline, D1 and D2 TSA signals are $16.5 \mathrm{~g}, 17.2 \mathrm{~g}$ and $19.15 \mathrm{~g}$ respectively. The abnormality of RMS of the TSA signals may indicate a problematic gearbox system and could be used as an initial indication factor of anomaly. However, RMS is tremendously affected by the shaft speed and load variation and thus RMS is not sufficient for comprehensive gear fault diagnosis. 


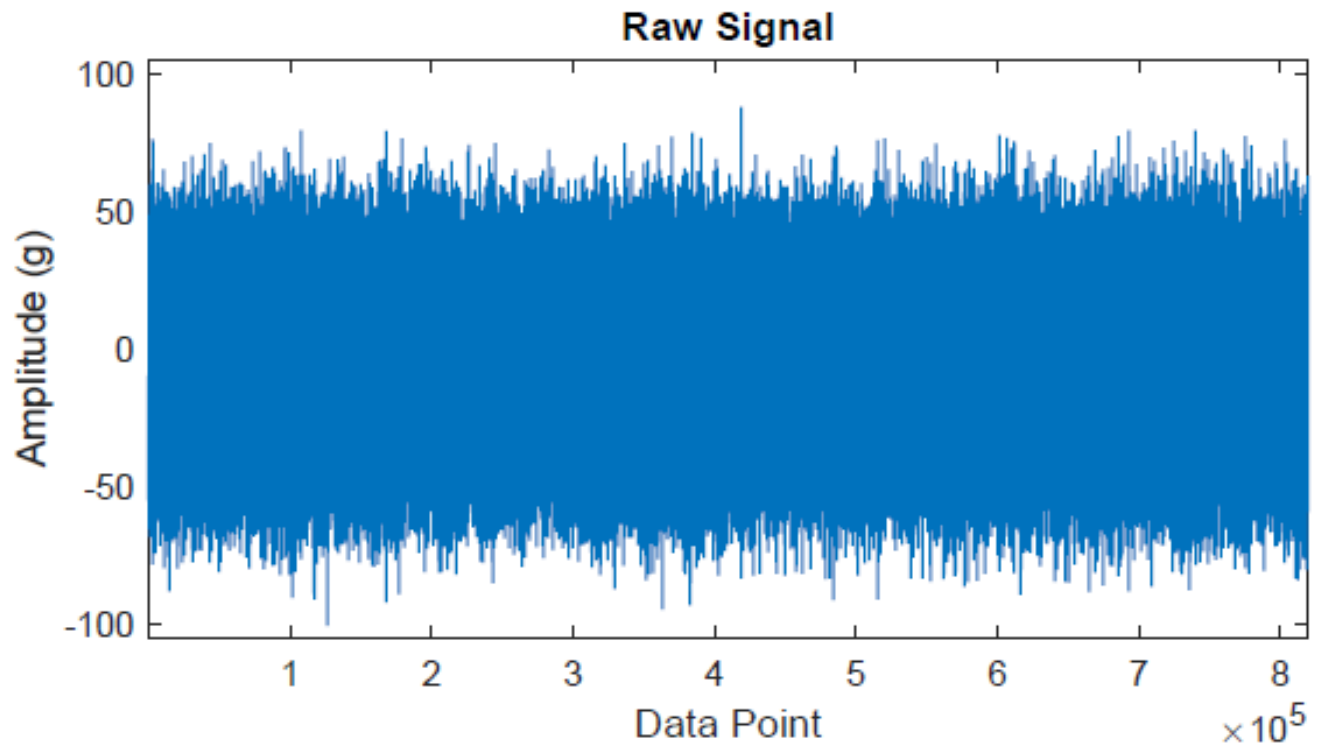

(a)

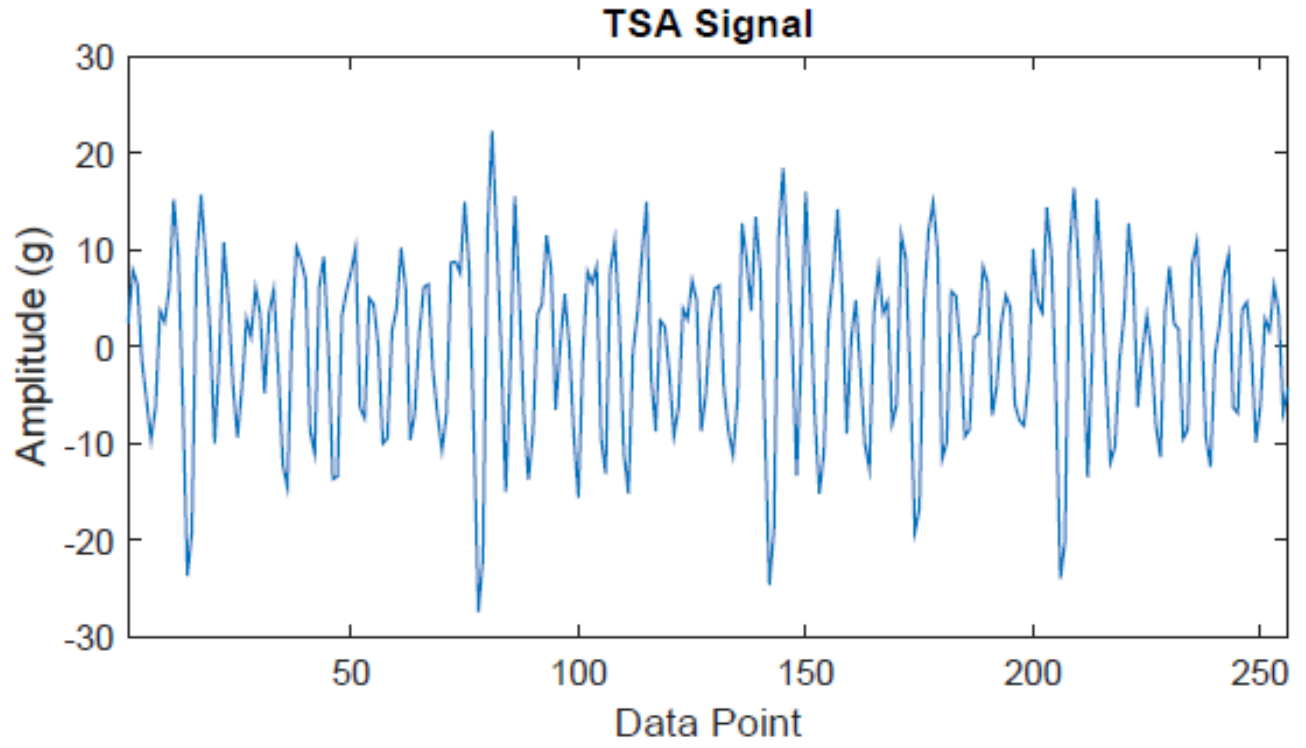

(b)

Figure 4-1 (a) Raw Signal (b) TSA signal. 

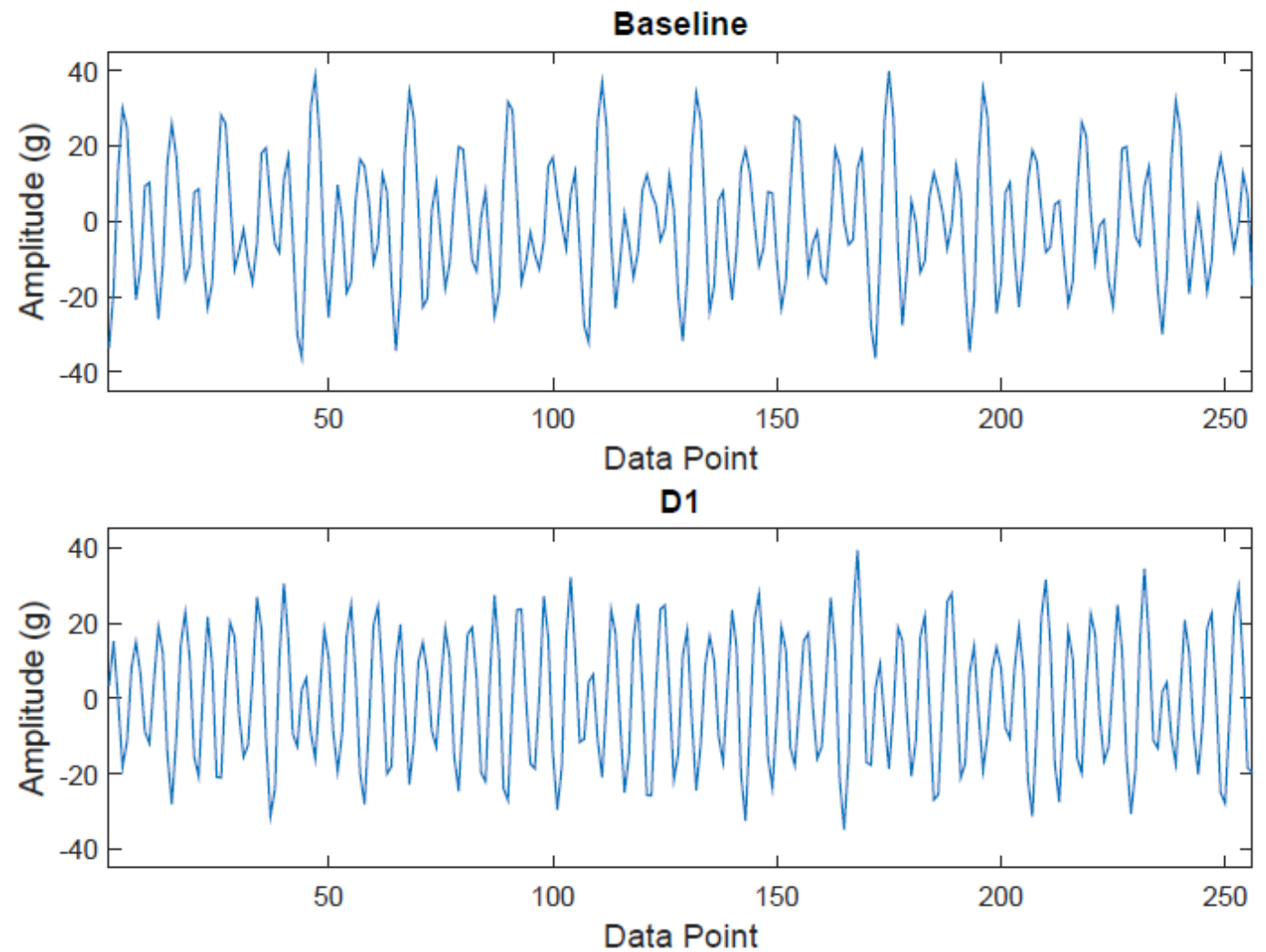

D2

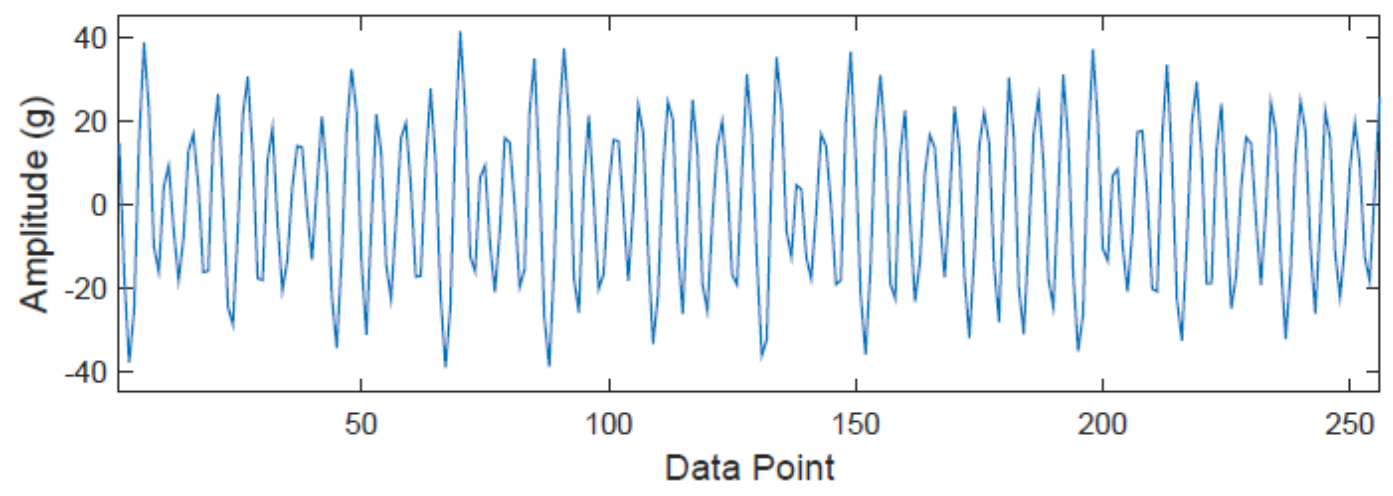

Figure 4-2 TSA signal at applied load \#3 of 1) Baseline test, 2) D1 test, 3) D2 test. 


\subsection{Amplitude and Phase Demodulation}

A pair of gears with identical profile under ideal operating conditions generate vibrations that contain the fundamental and several harmonics of tooth meshing frequency $N f_{r}$, where $N$ is the number of teeth of a gear and $f_{r}$ is the gear rotation frequency [31]. The vibrations can be estimated with a model:

$$
X(t)=\sum_{m=0}^{M} X_{m} \cos \left(2 \pi N f_{r} t+\phi_{m}\right)
$$

where $M$ is the number of teeth mesh harmonics considered.

If a local gear defect is developed, due to tooth stiffness variation, the gear mesh vibrations are modulated. The amplitude and phase modulation can be represented by a function $a_{m}(t)$ and $b_{m}(t)$ respectively:

$$
\begin{aligned}
& a_{m}(t)=\sum_{n=0}^{N} A_{m n} \cos \left(2 \pi n f_{r} t+\alpha_{m n}\right) \\
& b_{m}(t)=\sum_{n=0}^{N} B_{m n} \cos \left(2 \pi n f_{r} t+\beta_{m n}\right)
\end{aligned}
$$

Then the modulated vibration can be expressed as

$$
X(t)=\sum_{m=0}^{M} X_{m}\left(1+a_{m}(t)\right) \cos \left(2 \pi m N f_{r} t+\phi_{m}+b_{m}(t)\right)
$$

The amplitude demodulation of $X(t)$ can be obtained by determining the absolute magnitude of the analytic signal $z(t)$, while phase demodulation can be achieved by 
removing the initial phase angle from $z(t) . z(t)$ is determined from Hilbert transform:

$$
z(t)=X(t)-j H(X(t))
$$

Therefore, the analytic signals of $X(t)$ can be expressed as $z(t)$

$$
z(t)=\sum_{m=0}^{M} X_{m}\left(1+a_{m}(t)\right) \exp \left(j\left(2 \pi m N f_{r} t+\phi_{m}+b_{m}(t)\right)\right)
$$

The primary idea of implementing Hilbert transform is to process phase demodulation in a relative simple method. Based on equation 4.6, the phase of a function can be obtained by the angle between the real and imaginary value of the complex exponential function. Then, Hilbert transform plays an important role in obtaining the complex exponential function, or analytic signals. With the analytic signals, the amplitude demodulation can also be determined. Amplitude modulating signal $X_{m}\left(1+a_{m}(t)\right)$ can be obtained by taking the absolute amplitude of the Hilbert transform of modulated signals, i.e., TSA signal. Hilbert transform can be determined in MatLab with the function hilbert. The principle of phase demodulation to obtain $b_{m}(t)$ involves removing the initial phase angle, $\left(2 \pi m N f_{r} t+\right.$ $\left.\phi_{m}\right)$, from the instantaneous phase of the analytical function $z(t)$. The initial phase angle is a linear function with a slope of $2 \pi m N f_{r}$ and an interception of $\phi_{m}$. In practical implementation, the linear offset $2 \pi m N f_{r} t+\phi_{m}$ can be estimated by linear least-square fit [85]. The modulation term $b_{m}(t)$ is calculated by subtracting the estimated linear offset from the instantaneous phase.

The amplitude and phase demodulation of TSA signals are shown in Figure 4-3 and Figure 4-4 respectively. Amplitude demodulation determines the amplitude of the modulating 
components while phase demodulation shows the phase variation of the modulating terms. The red curves in Figure 4-3 are the demodulated envelopes of TSA signals and they are oscillating at around $25 \mathrm{~g}$ for all gear conditions. General observation implies that the pattern of the envelopes is similar for the three TSA signals. The figure shows that baseline envelope has more distinctly large jumps whereas the envelopes of D1 and D2 show fewer large jumps due to magnitude increase of small jumps. The RMS of amplitude demodulation increases with the size of spall. The baseline envelope has RMS value of 23.8 and it rises to 24.3 and 27.4 respectively when the spall is developed from D1 to D2.

The phase demodulation outputs the instantaneous phase of major sidebands according to the principle of phase demodulation. The phase variation, on the other hand, is attenuated with the presence of a spall. In Figure 4-4, the phase-change of the demodulated phase of baseline signal is dramatically reduced by the spall. The RMS of baseline phase is $2.03 \mathrm{rad}$ whereas the RMS of D1 and D2 phase are both approximately 0.47 rad. The reduction of overall phase suggests that the spall produces a phase function which has opposite direction and spans across the entire time period. 
(1) Baseline

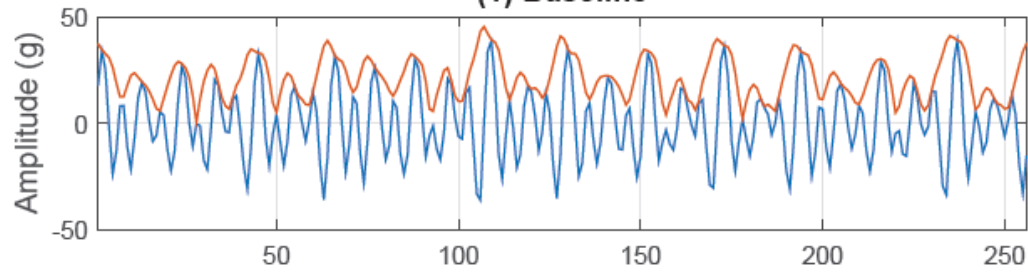

(2) D1

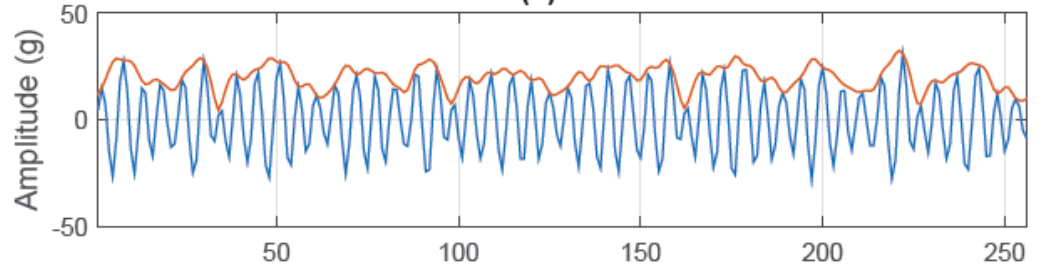

(3) D2

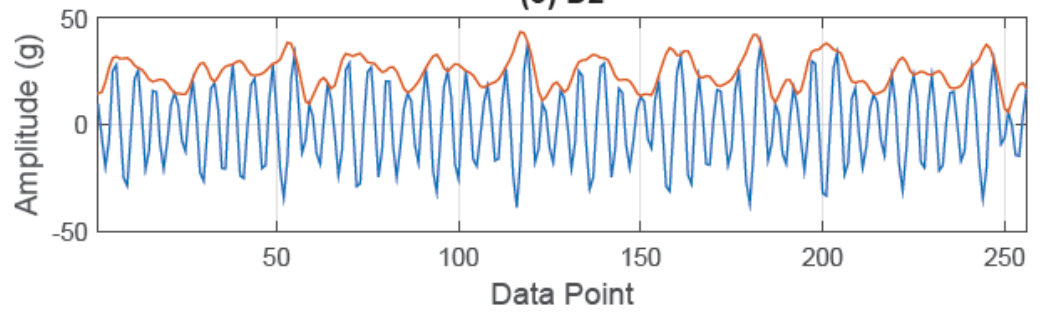

Figure 4-3 Amplitude Demodulation of TSA signals

(1) Baseline

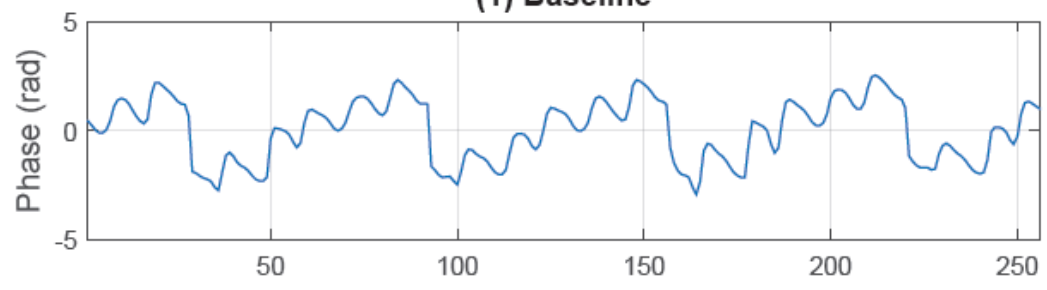

(2) D1

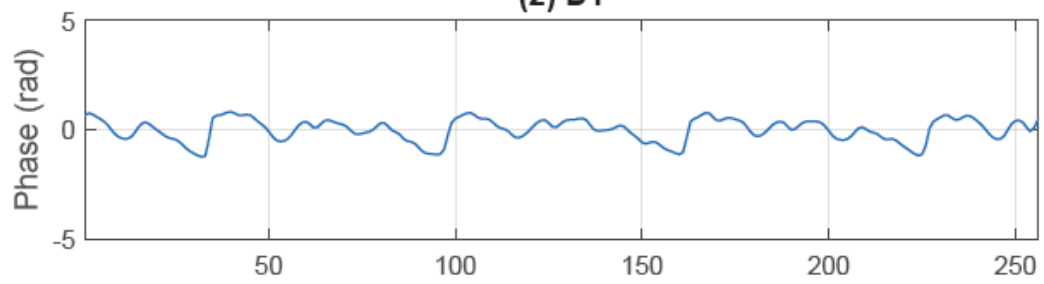

(3) D2

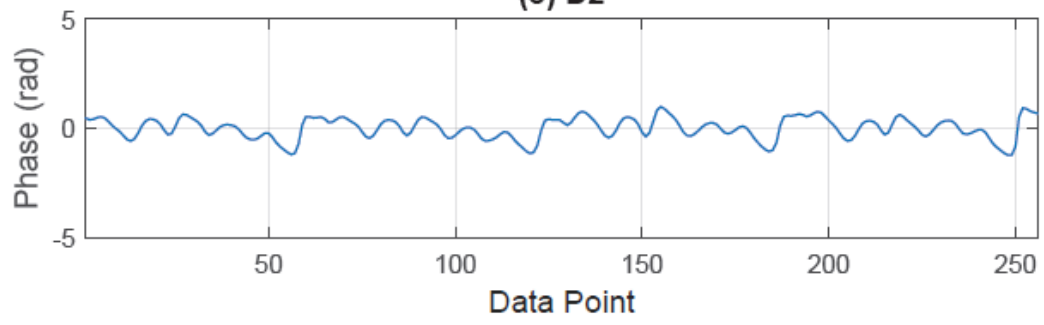

Figure 4-4 Phase demodulation of TSA signals 


\subsection{Fast Fourier Transform}

Fast Fourier Transform (FFT) is an algorithm to implement the Discrete Fourier Transform, which is defined as:

$$
X(m)=\sum_{n=0}^{N-1} x(n) e^{-j 2 \pi m n / N}
$$

FFT increases the computing speed of DFT by reducing the number of computations from $\mathrm{N} 2$ to $2 \mathrm{~N} \log 2 \mathrm{~N}$ for $\mathrm{N}$ data [86]. The computation of FFT signal is accomplished by using the Matlab built-in function $f f t$. Traditional spectrum analysis involves defining the frequency values on the abscissa of a spectrum with the sampling frequency. This approach does not apply to the time synchronous averaged signals because the equivalent sampling frequencies are constantly varied during the process of resampling so that the original sampling frequency can no longer define the frequency values. In order to accommodate the effect of resampling, order analysis is applied in the spectrum to represent frequency components in terms of the rotation frequency of the selected shaft. On the other hand, the $1^{\text {st }}$ harmonic of GMF in the order spectrum is equivalent to the number of teeth of the target gear and the $2^{\text {nd }}$ harmonic of GMF is twice as that number, and so on.

As shown in baseline TSA spectrum in Figure 4-5, the frequency values are expressed in terms of shaft speed orders instead of Hertz. The harmonics of GMF are 24, 48, 72 and 96, which are consistent with the physical property of the 24 teeth-gear. It is indicated that the major sidebands appear between the fundamental (the $1^{\text {st }}$ ) and the dominant (the $2^{\text {nd }}$ ) harmonic of GMF. It also demonstrates that the $2^{\text {nd }}$ harmonic of GMF is the carrier 
frequency in the modulation process because the major sidebands are the smeared frequencies of the $2^{\text {nd }}$ harmonic of GMF in the lower region. A major discovery from the spectrum of baseline, D1 and D2 in Figure 4-5 is the magnitude variation of the $1^{\text {st }}$ and the $2^{\text {nd }}$ harmonic of GMF. The spall raises the energy of the $2^{\text {nd }}$ harmonic of GMF while it lowers the magnitude of the $1^{\text {st }}$ harmonic of GMF. As it is shown, the magnitude of the $1^{\text {st }}$ harmonic of GMF of the baseline, D1 and D2 spectra are $12.17 \mathrm{~g}, 4.39 \mathrm{~g}$ and $6.33 \mathrm{~g}$ respectively. The energy of $1^{\text {st }}$ harmonic of GMF decreases $64 \%$ with the occurrence of D1 spall while D2 spall reduces the energy of $1^{\text {st }}$ harmonic of GMF by $48 \%$. The magnitude of the $2^{\text {nd }}$ harmonic of GMF increases from $15.58 \mathrm{~g}$ to $20.46 \mathrm{~g}$ ( $31 \%$ increase) when D1 spall is present and it reaches $23.05 \mathrm{~g}$ ( $48 \%$ increase) as the spall size propagates to $\mathrm{D} 2$. 


\section{(1) Baseline}

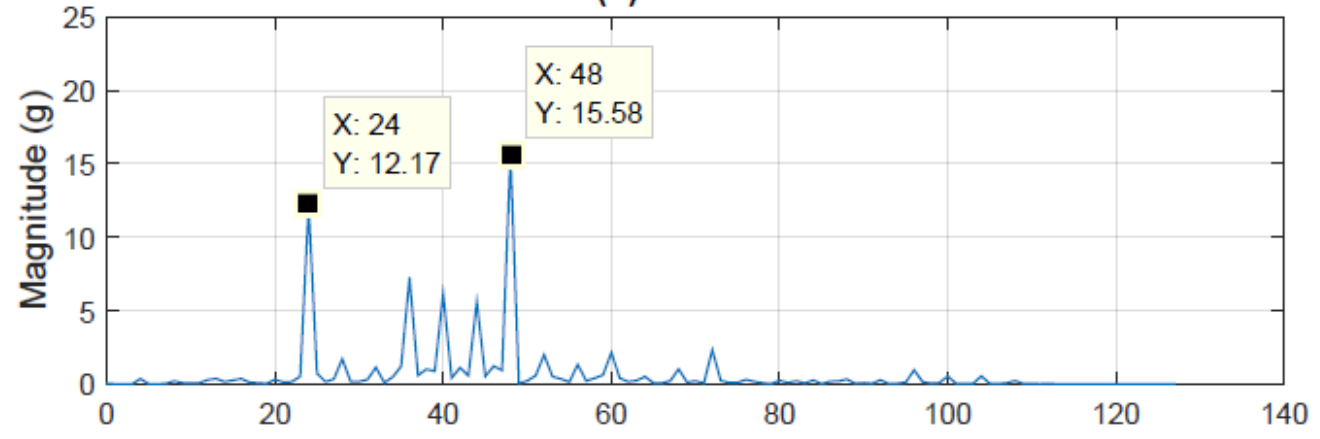

(2) D1

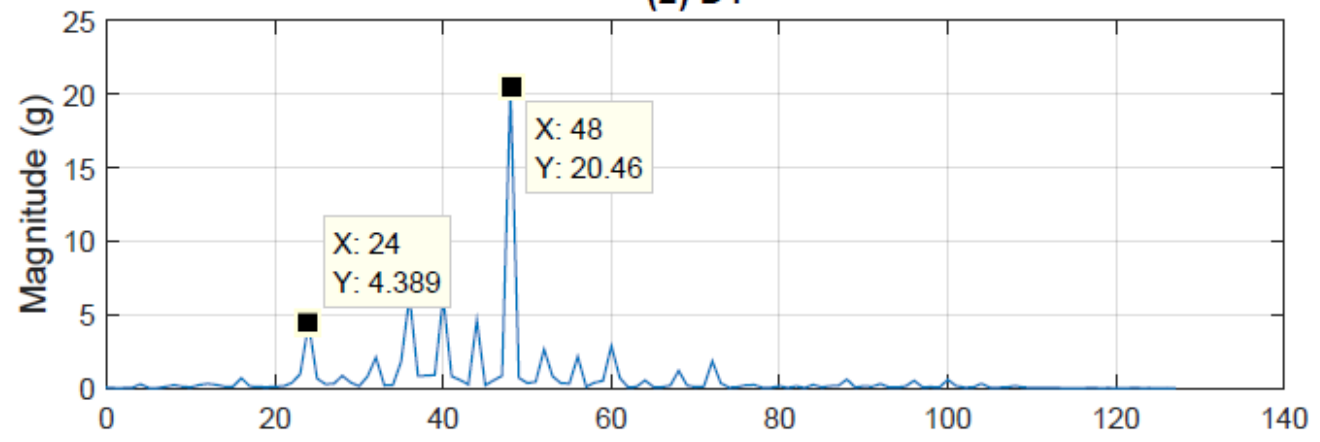

(3) D2

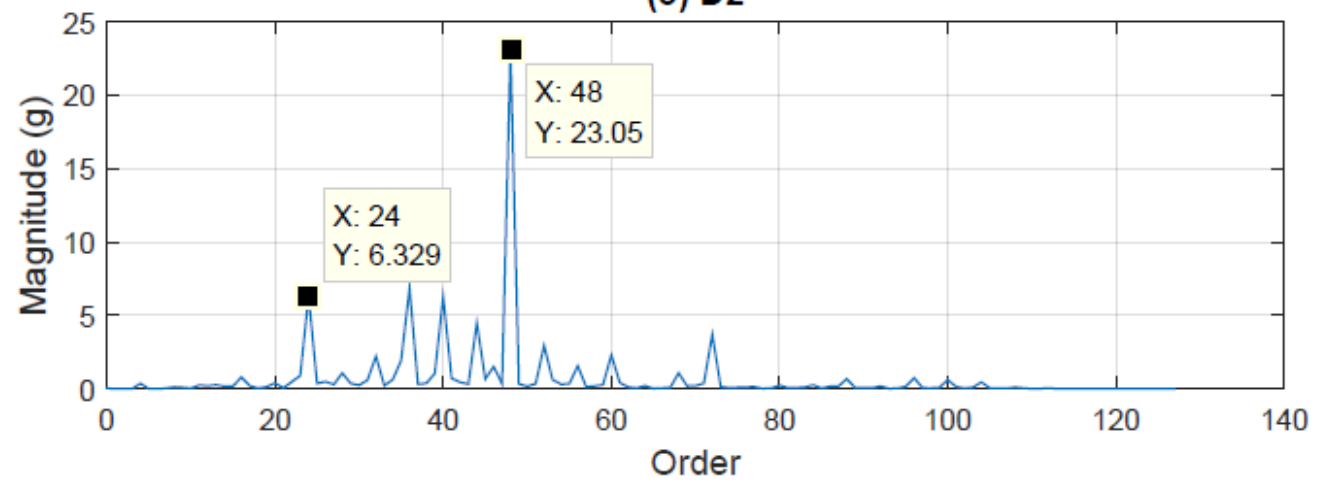

Figure 4-5 TSA spectrum: 1) baseline test, 2) D1 test, 3) D2 test 


\subsection{Continuous Wavelet Transform (CWT)}

The continuous wavelet transform is defined as follows [51]:

$$
C W T_{x}^{\psi}(\tau, s)=\Psi_{x}^{\psi}(\tau, s)=\frac{1}{\sqrt{s}} \int x(t) \psi^{*}\left(\frac{t-\tau}{s}\right) d t
$$

where $\tau$ and s refer to translation and scale respectively. $\psi(t)$ is called mother wavelet. $\psi^{*}(t)$ denotes the complex conjugate of the mother wavelet function. The mother wavelet is the main window function that is used to derive other window functions by multiplying the mother wavelet from low scale to high scale. Continuous wavelet transform can be computed in a fast way using Fourier Transform algorithm. According to Equation 4.11, CWT is the time convolution of the time signal and scaled mother wavelets. Convolution in time domain is equivalent to multiplication in frequency domain. Then, Equation 4.11 can be transformed into following equation [87]:

$$
C W T(\tau, s)=\sqrt{s} F^{-1}\left[X(f) W_{s}(f)\right]
$$

$X(f)$ and $W_{s}(f)$ are the FFT of $x(t)$ and $\psi^{*}\left(\frac{t-\tau}{s}\right)$ respectively. $F^{-1}$ is the inverse Fourier Transform operator. In frequency domain, $X(f)$ requires to be computed only once and $W_{s}(f)$ needs to be calculated for all scales, whereas in time domain both $x(t)$ and $\psi^{*}\left(\frac{t-\tau}{s}\right)$ are computed on all scales.

A wavelet map is a time-frequency representation which is generated by taking the absolute amplitude of wavelet coefficients of TSA signals. Figure 4-6 illustrates 3 wavelet maps of the TSA signals of gear F2 in 3 different health conditions respectively. High spectral 
energy occurs and concentrates at the $2^{\text {nd }}$ harmonic of GMF (48 order) and strip-shape energy pattern is formed while a much lower energy pattern spans across the fundamental GMF (24 order) in the baseline wavelet map. The formation of energy strip is due to residue energy of the $2^{\text {nd }}$ harmonic of GMF filtered by a successive moving wavelet filters overlapping with spectral smearing energy of sidebands from order 36 to order 52 . The baseline TSA signal have 48 peaks (Figure 4-6) whereas there are 12 distinctive energy strips spaced evenly at around the dominant GMF. The reason is due to the fact that the time resolution employed in the wavelet transform is not fine enough and the main purpose was to obtain better frequency resolution. With occurrence of a spall, the energy of the $2^{\text {nd }}$ harmonic of GMF is strengthened with the sacrifice of the power of the $1^{\text {st }}$ harmonic of GMF, which cross validates the results obtained from spectrum analysis. Furthermore, the area of each energy strip is enlarged and some of the energy strips are merged due to the gear defect. Wavelet map of D1 has a large energy pattern near time location of 50 (as circled in red) and similar energy pattern is observed in the wavelet map of D2 at time location of 200. As mentioned above, the spall introduces modulation effect that increases the amplitude of 'the three small jumps' of the baseline TSA signal. Since the time resolution of wavelet transform at a particular frequency band is fixed, two high energy peaks are detected as one large energy spot in the wavelet map. Therefore, it indicates that the local formation of high energy pattern is not caused by local transient excitation generated by the spall but is a result of modulation that affects the vibration signatures globally. The wavelet map provides intuitive representation for gearboxes fault diagnosis, which can be used as a preliminary factor in the early stage of decision making. 

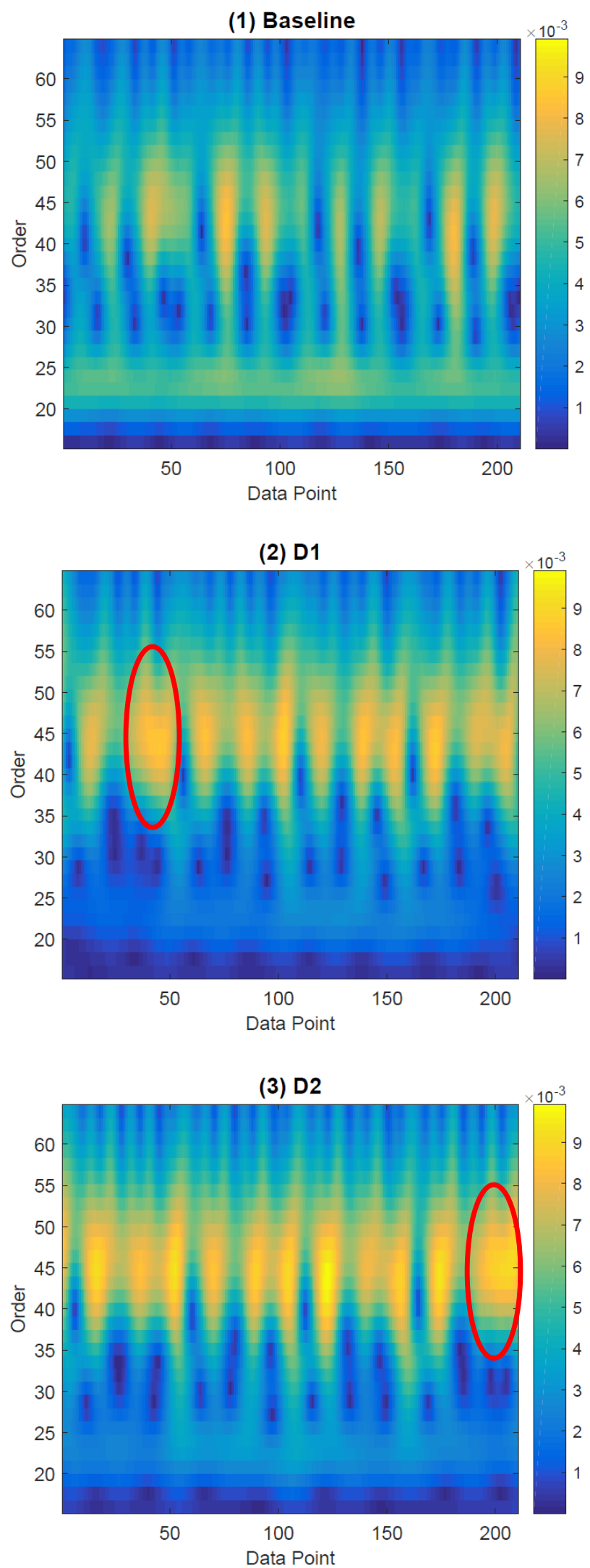

Figure 4-6 Wavelet map of TSA signals: 1) baseline test, 2) D1 test, 3) D2 test 


\subsection{Enhanced Empirical Mode Decomposition (EMD) and Teager's Kaiser Energy Operator (TKEO)}

EMD is a signal decomposition technique that decomposes a signal into a finite number of IFMs, where instantaneous frequency is computed effectively. As indicated by Huang [68], for a function to provide meaningful instantaneous frequency, it should hold two conditions: (1) symmetric with respect to the local zero mean, (2) has the same numbers of zero crossings and extrema, i.e., maxima and minima. He then proposed the IMFs that approximately meet the above conditions. He defines another two conditions for IMFs: (1) in the whole data set, the number of extrema and the number of zero crossings must either be equal or differ at most by one; and (2) at any point, the mean value of the envelope defined by the local maxima and the envelope defined by the local minima is zero. EMD is developed to obtain IMFs to satisfy those conditions. The algorithm is called sifting process and it is comprehensively described in [68]. Some main problems of EMD are addressed by Rato [88], including the effect of extrema locations, end effects and sifting stopping condition; while he proposed some solutions to alleviate those problems. Then the enhanced EMD is applied to EEG signals for performance evaluation and the outcome was extraordinary. The enhanced EMD along with TKEO is first used for gearbox fault diagnosis in this thesis. The detailed algorithm is demonstrated in the following discussion with illustrations. 
EMD decomposes a signal into several IMFs and residue:

$$
x(t)=\sum_{i=1}^{n} I M F_{i}(t)+r_{n}(t)
$$

where $I M F_{i}$ is the $\mathrm{i}^{\text {th }} \mathrm{IMF}$ and $r_{n}$ is the residue.

IMF is obtained with an iterative process followed by:

(1) set $h(t)=x(t)$, determine the maxima $M_{j}$ and minima $m_{j}$ of $h_{1}(t), j=$ $1,2,3, \ldots k$

(2) use parabolic interpolation to estimate better location of extrema values [88];

(3) extrapolate a maximum and a minimum for both ends;

- With the first maximum $M_{1}$ and first minimum $m_{1}$, determine the corresponding time location $T_{1}$ and $t_{1}$;

- The extrapolated maximum to the beginning of the signal, $M_{0}=M_{1}$ at time location $T_{0}=-t_{1}$, while a new minimum $m_{0}=m_{1}$ at time $t_{0}=$ $-T_{1}$

- Similar extrapolation is applied to obtain a maximum and a minimum at the end of the signal;

(4) estimate the upper and lower envelopes, $e_{u}(t)$ and $e_{l}(t)$ with cubic spine interpolation;

(5) a basis function is computed as $B(t)=\left(e_{u}(t)+e_{l}(t)\right) / 2$;

(6) determine if the stopping criterion is satisfied;

The stopping criterion is a comparison between a resolution factor and a predefined resolution. The resolution factor is given by the following equation [88]: 


$$
\text { Resolution Factor }=10 \log _{10}\left(\frac{E[h(t)]}{E[B(t)]}\right)
$$

where $E[]$ is the energy operator, $h(t)$ is the signal of the sifting process and $B(t)$ is the basis function. A predefined resolution is chosen to be $50 \mathrm{~dB}$ which is sufficient for machinery vibration signals. If the resolution factor exceeds the predefined resolution, then the sifting process stops;

(7) stopping criterion is satisfied: $I M F_{1}=h(t)-B(t)$; stopping criterion is not satisfied: $h(t)=h(t)-B(t)$ and repeat steps 2-7;

(8) determine the residue $r_{1}=x(t)-I M F_{1}$;

(9) check if the residue can be decomposed into IMFs;

if residue has more than one local extreme, treat $r_{1}$ as the original signal $x(t)$ and repeat the steps 1-9.

If residue has at most one local extreme, the EMD decomposition stops

TKEO was first introduced by Kaiser [89] as a non-linear operator which is capable of tracking linear oscillator and thus measure the modulation energy. TKEO was further applied to analyze speech signals through amplitude and frequency demodulation by calculating the instantaneous amplitude and frequency [90] [91]. The application of TKEO is widely extended to the engineering field, where bearing faults are diagnosed with the assistance of TKEO [92]. The discrete-time TKEO is defined as [93]:

$$
\psi\left[(x(t)]=[\dot{x}(t)]^{2}-x(t) \ddot{x}(t)\right.
$$

where $\psi$ is the energy operator, $\dot{x}(t)$ and $\ddot{x}(t)$ are the first and second time derivatives of 
$x(t)$ respectively. The $\dot{x}(t)$ and $\ddot{x}(t)$ can be approximated by time difference in discretetime domain and hence Equation 4.12 becomes

$$
\psi\left[(x(t)]=x^{2}(n)-x(n+1) x(n-1)\right.
$$

The instantaneous frequency $f(n)$ and instantaneous amplitude $|A(n)|$ can be calculated using discrete-time energy separation algorithm 1 (DESA-1) [93]:

$$
\begin{gathered}
f(n)=\arccos \left(1-\frac{\psi[x(n)-x(n-1)]}{4 \psi[x(n)]}\right) \\
|A(n)|=\sqrt{\frac{\psi[x(n)]}{1-\left(1-\frac{\psi[x(n)-x(n-1)]}{4 \psi[x(n)]}\right)^{2}}}
\end{gathered}
$$

A simulated signal is used to demonstrate the effectiveness of the enhanced EMD and TKEO decomposition. The signal, $x(t)$, consists of two parts, $x_{1}=\cos (2 \pi 40 t+$ $0.5 \sin (2 \pi 20 t))$ and $x_{2}=\sin (2 \pi 160 t) \cdot x_{1}$ has a carrier frequency of $40 \mathrm{~Hz}$ which is frequency demodulated by a $20 \mathrm{~Hz}$ sine wave, while $x_{2}$ is a single sine wave oscillated at $160 \mathrm{~Hz}$. The signal is shown in Figure 4-7. The extrapolation described in EMD step (3) is intended to reduce the end effect due to lack of data at both ends during cubic spline interpolation, which causes divergence at both ends of the interpolated envelopes. As it shows in Figure 4-8, divergence occurs at the beginning and at the end of the envelopes obtained from original extreme; whereas extreme extrapolation eliminates the end effect. The simulated signal is decomposed into two IMFs and a residue (Figure 4-9). The first IMF, IMF 1 contains high frequency component while $\mathrm{IMF}_{2}$ appears to be a low frequency 
signal. This is due to the sifting process where low frequency components are eliminated while high frequency components are remained in the decomposed signal. Therefore, IMFs are outputted in a frequency order from high to low. So EMD is equivalent to a bank of filters. TKEO and DESA-1 are applied to each IMF to examine the instantaneous frequency and instantaneous amplitude. Figure 4-10 shows that instantaneous frequency of $\mathrm{IMF}_{1}$ spans across the $160 \mathrm{~Hz}$ for the entire time span, while the instantaneous amplitude of $\mathrm{IMF}_{1}$ is precisely located at magnitude 1 (Figure 4-11). Both instantaneous frequency and instantaneous amplitude indicate that $\mathrm{IMF}_{1}$ is the second parts of the signal $\left(x_{2}\right)$. It is observed that the instantaneous frequency of $\mathrm{IMF}_{2}$ oscillates around $40 \mathrm{~Hz}$, suggesting frequency modulation of the component $40 \mathrm{~Hz}$. The spectrum of the instantaneous frequency of $\mathrm{IMF}_{2}$ (Figure 4-12) confirms that the modulating frequency is $20 \mathrm{~Hz}$. It is worthy to mention that the $20 \mathrm{~Hz}$ shown on the spectrum is actually $20.48 \mathrm{~Hz}$ with the magnitude of 0.48 . The deviation of frequency value is caused by discrete-time approximation of DESA-1 while the magnitude discrepancy is due to FFT error. The instantaneous amplitude of $\mathrm{IMF}_{2}$ also indicates the occurrence of amplitude modulation. The example has proven that EMD associated with TKEO is an efficient tool of obtaining demodulation features of a signal and hence, this technique has been employed to analyze the gearbox signals. 


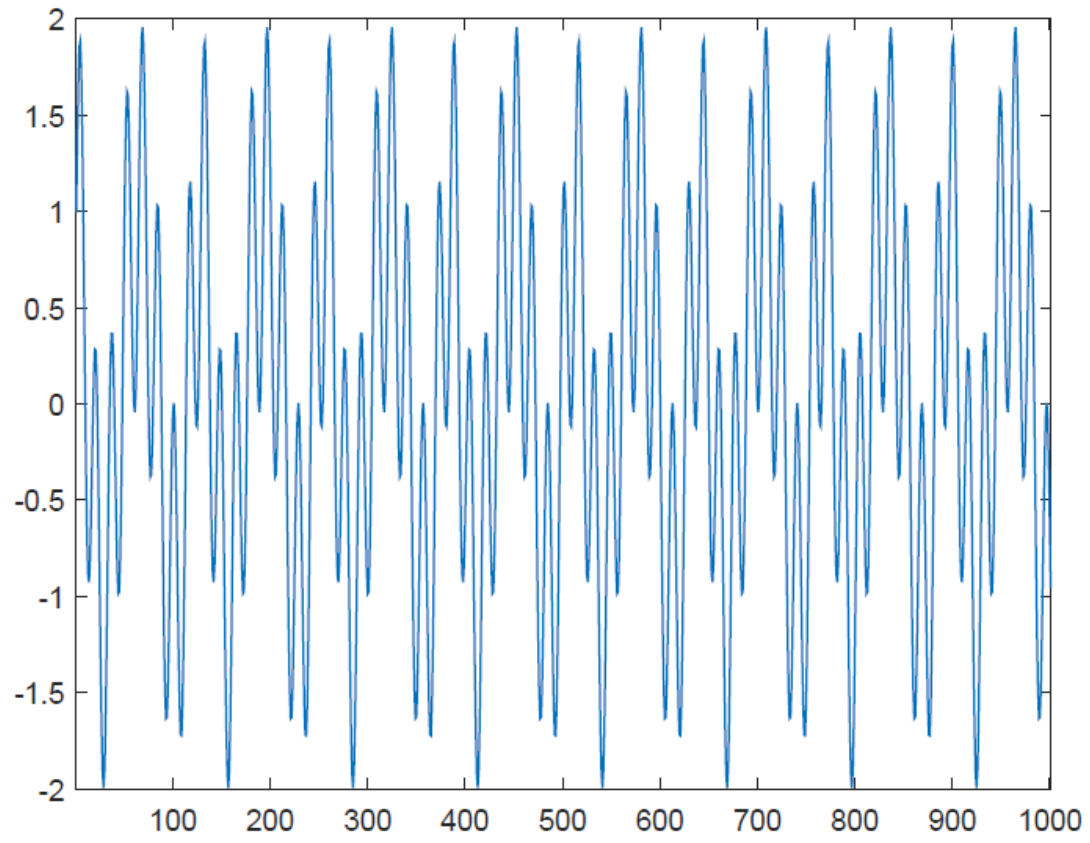

Figure 4-7 The simulated signal 


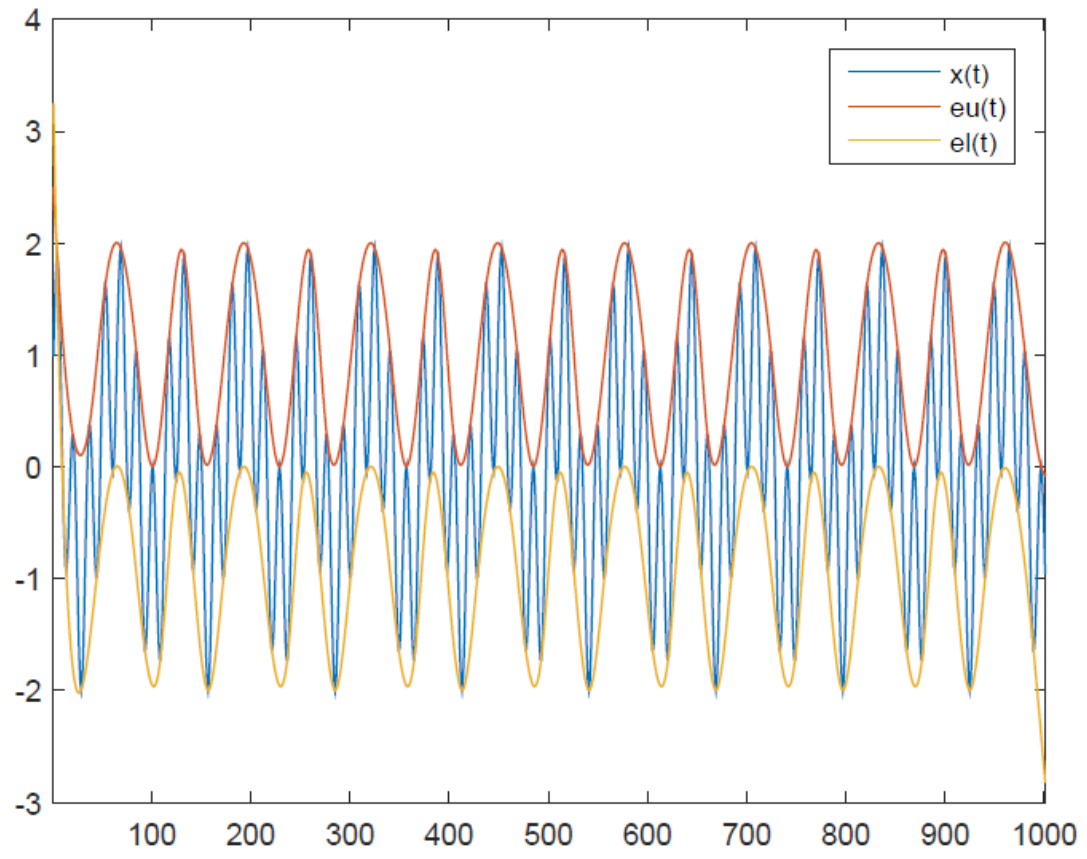

(1)

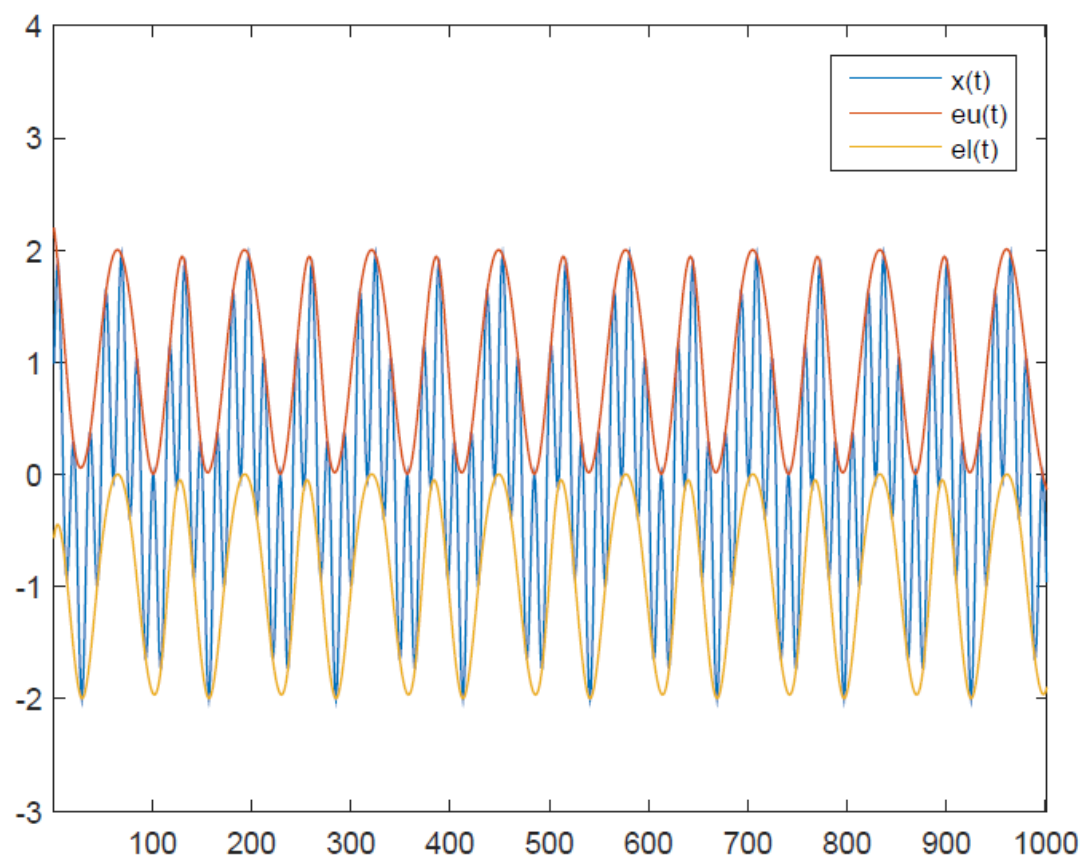

(2)

Figure 4-8 End effect illustration. Envelopes 1) without extrapolation 2) with extrapolation 

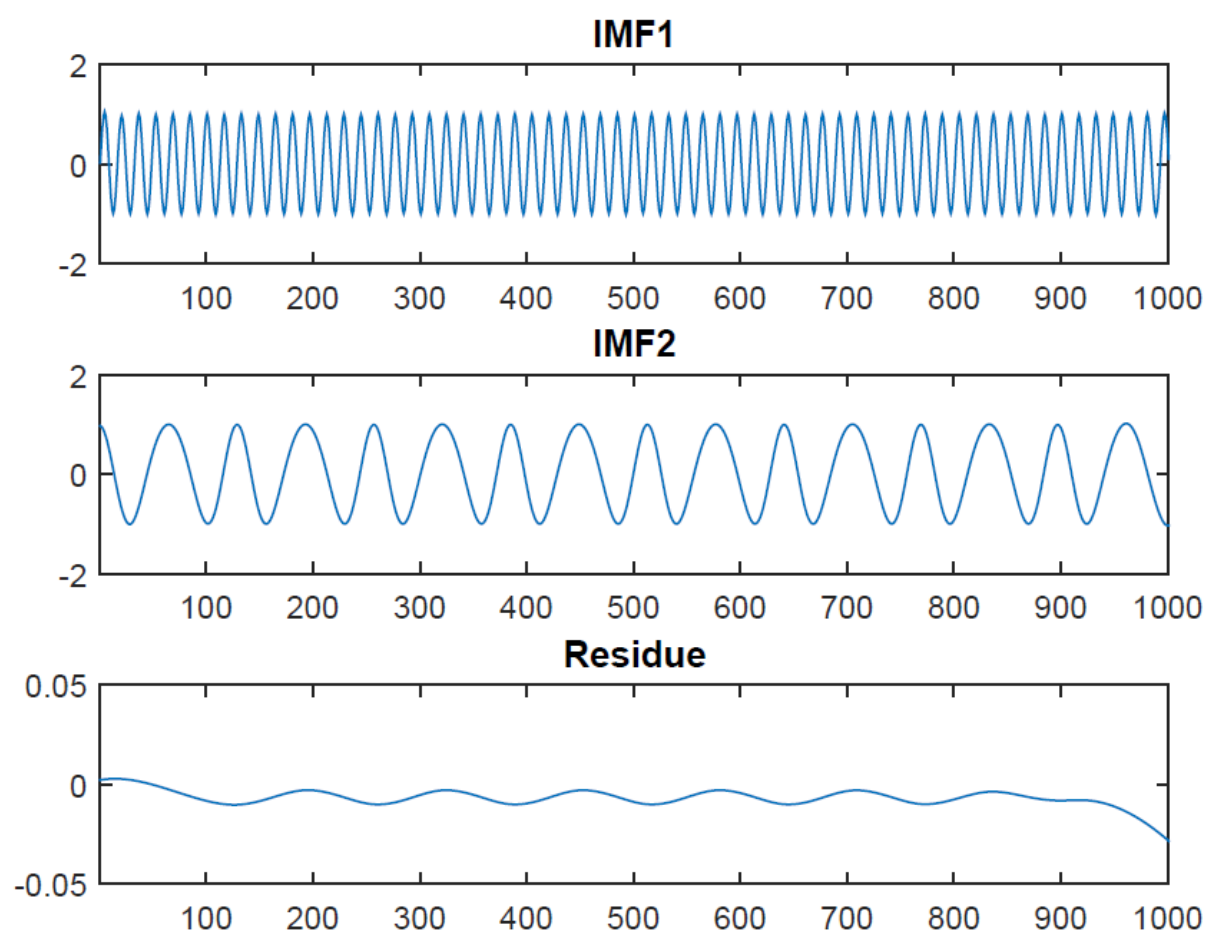

Figure 4-9 EMD decomposition of the simulated signal
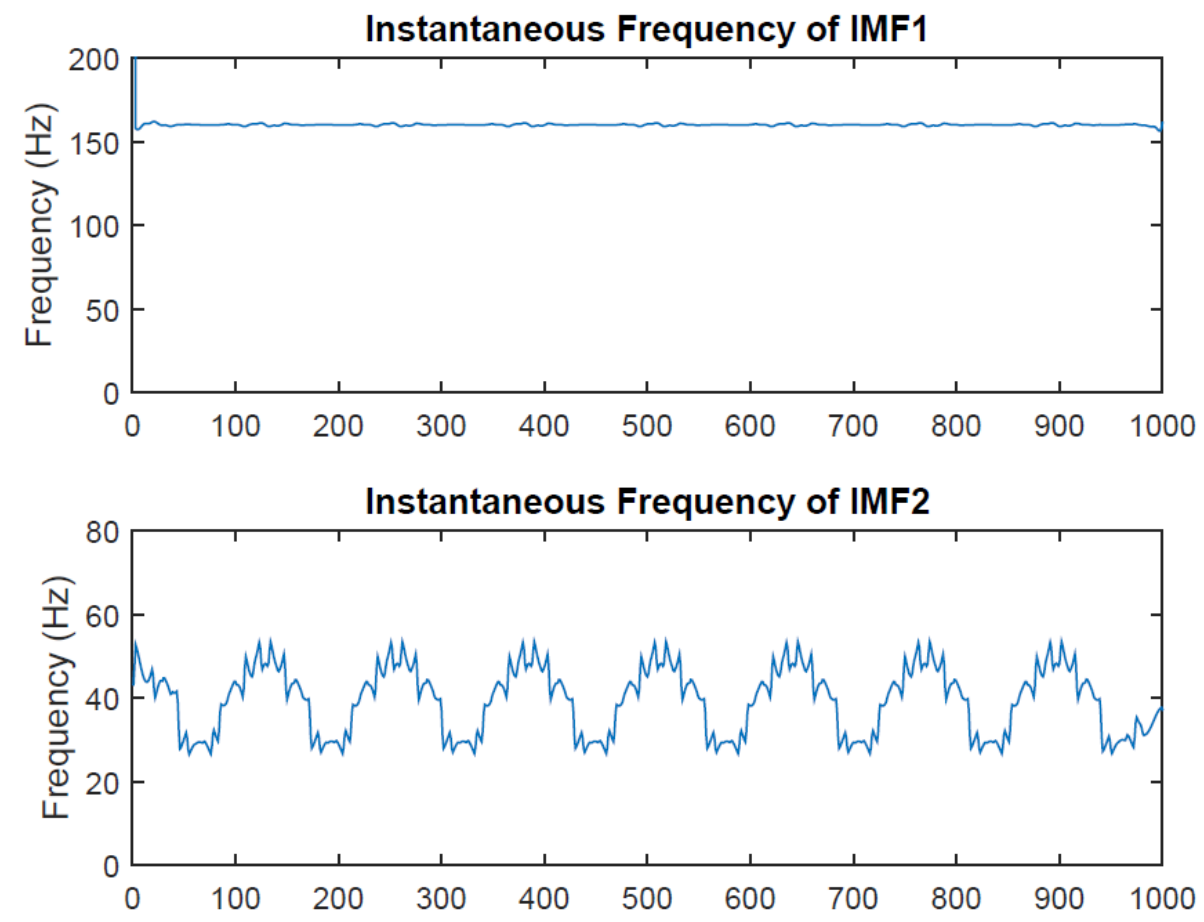

Figure 4-10 Instantaneous frequency of $\mathrm{IMF}_{1}$ and $\mathrm{IMF}_{2}$ 

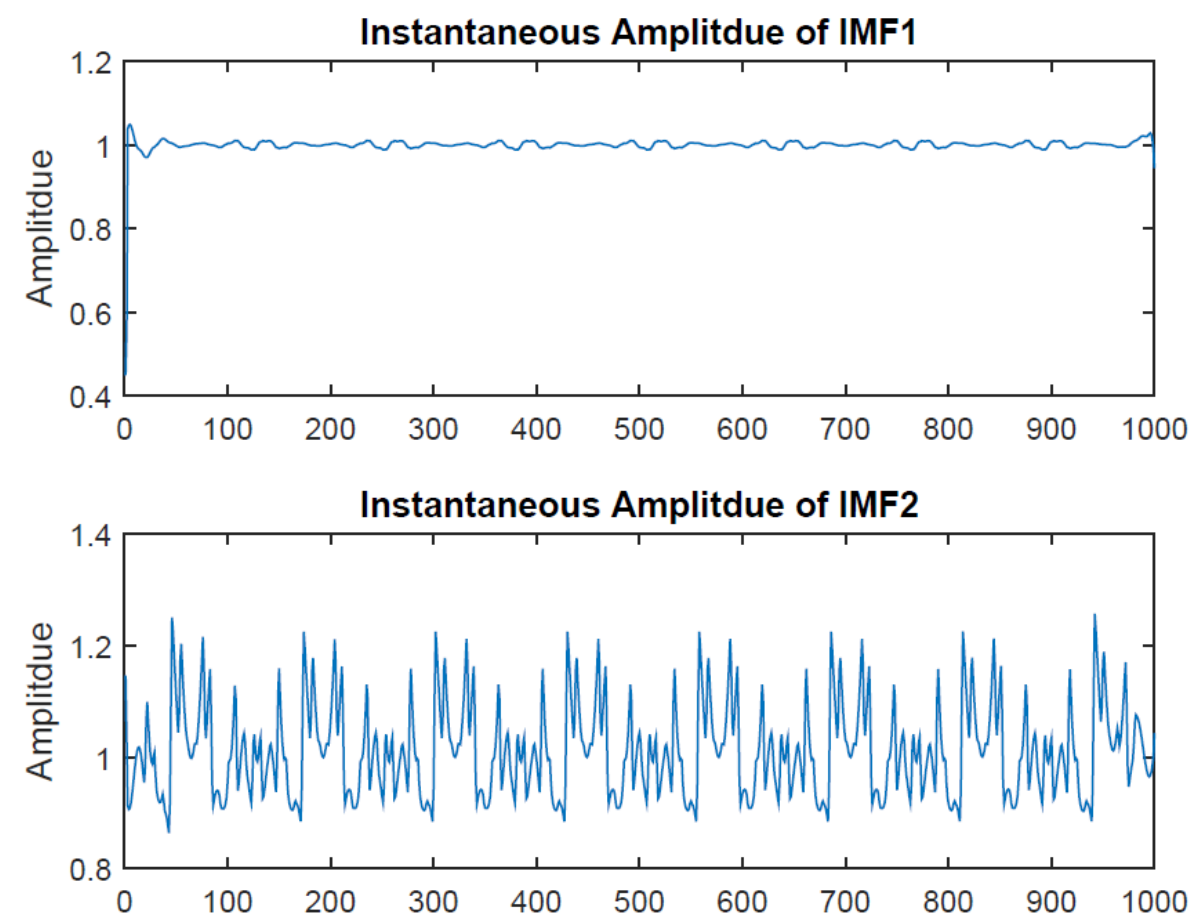

Figure 4-11 Instantaneous amplitude of $\mathrm{IMF}_{1}$ and $\mathrm{IMF}_{2}$

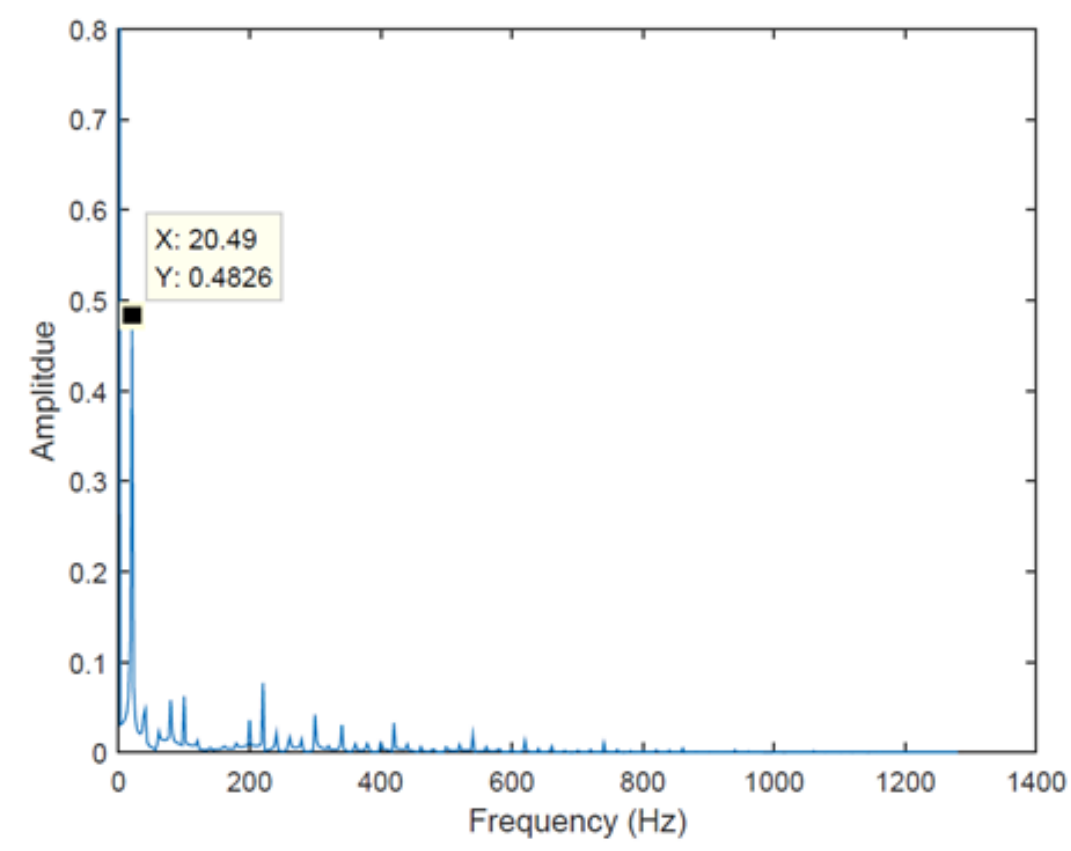

Figure 4-12 Spectrum of instantaneous frequency of $\mathrm{IMF}_{2}$ 
EMD decomposes a baseline TSA signal into 4 IMFs, as shown in Figure 4-13. The IMFs inherit the property of EMD that IMFs are outputted in a frequency order from high to low. Based on the time history of IMFs, the number of peaks is a representation of frequency component. It can be determined from Figure 4-13 that the number of peaks of the $1^{\text {st }}$ IMF to the $4^{\text {th }}$ IMF are $48,24,12$ and 4 respectively. The frequency components of the $1^{\text {st }}$ IMF include the $2^{\text {nd }}$ harmonic of GMF and the associated sidebands since modulation appears at the $1^{\text {st }}$ IMF whose envelopes are oscillating at the sideband frequencies. The spectra of the 4 IMFs are plotted in Figure 4-14 and it shows that $1^{\text {st }}$ IMF contains the $2^{\text {nd }}$ harmonic of GMF and 3 lower sidebands along with low energy upper sidebands and higher harmonics of GMF. The spectrum of the $2^{\text {nd }}$ IMF has one large peak at the $1^{\text {st }}$ harmonic of GMF followed by a low amplitude modulated sideband. Modulation can also be observed from the varying envelope of the time history of the $2^{\text {nd }}$ IMF (Figure 4-13). The main spectral component of the $3^{\text {rd }}$ IMF is 12 order which is the $3^{\text {rd }}$ harmonic of the frequency component of the $4^{\text {th }}$ IMF, 4 order. 

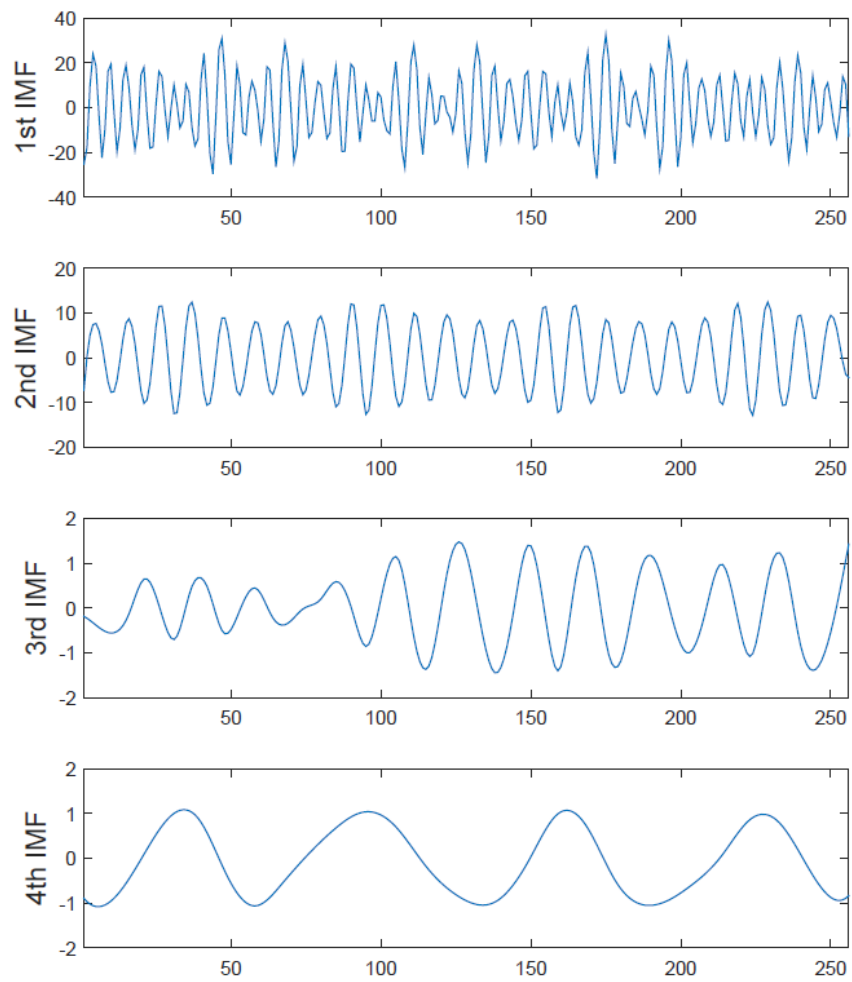

Figure 4-13 The first 4 IMFs of baseline TSA EMD decomposed signal
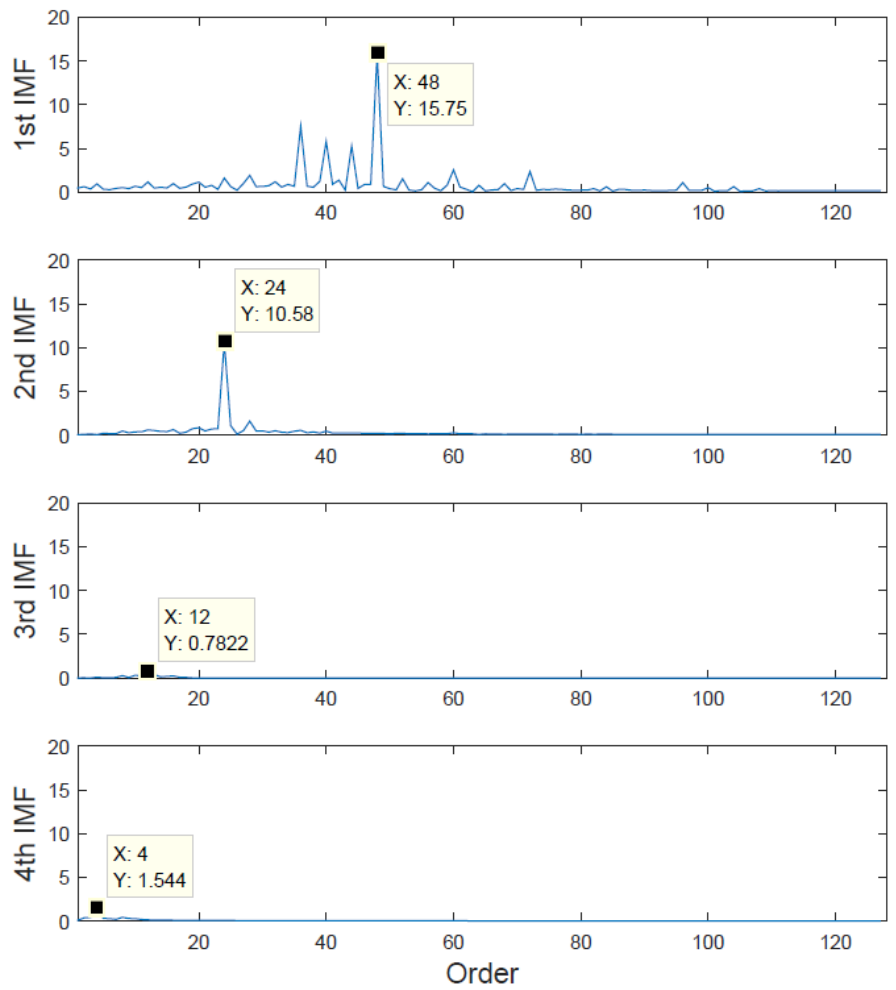

Figure 4-14 Spectrum of the 4 IMFs 
General modulation involves not only amplitude and phase modulation, but also frequency modulation. The characteristics of amplitude and phase modulation due to a spall have been extracted by the aforementioned techniques except for the frequency modulation. It is believed that the frequency modulated features are hidden in the $1^{\text {st }}$ harmonic of GMF because the $1^{\text {st }}$ harmonic of GMF is crucial to the spalling detection in this case. Since there is only one spall on a tooth of the gear F2, the spall generates excitation forces in each revolution of gear rotation which alters the phase of the $1^{\text {st }}$ harmonic of GMF. Previous discussed techniques lack the ability to extract the frequency modulated information of a specific frequency band. To this end, the enhanced EMD associated with TKEO is applied to TSA signals to extract instantaneous amplitude and frequency from the $1^{\text {st }}$ harmonic of GMF.

The instantaneous amplitude determined by the TKEO algorithm also demonstrates that spalling introducing modulation causes a decrease in the magnitude of the $1^{\text {st }}$ harmonic of GMF. As shown in Figure 4-15, the instantaneous amplitude curves are constructed by four large waves and the small consecutive ripples which are the result of discrete computation of TKEO. The average instantaneous amplitude of baseline signal is approximately $10 \mathrm{~g}$; whereas the average instantaneous amplitude of D1 and D2 signals are estimated to be $4 \mathrm{~g}$ and $5 \mathrm{~g}$ respectively. These conclusive results are consistent with those obtained from previous techniques. Figure 4-16 explores the characteristics of frequency modulation of the $1^{\text {st }}$ harmonic of GMF. The instantaneous frequency of the $2^{\text {nd }}$ IMF is oscillating around the $1^{\text {st }}$ GMF with an average amplitude of $6 \mathrm{~g}$, indicating frequency modulation. As D1 spall is developed on a tooth surface, the average amplitude of instantaneous frequency is increased to about $13 \mathrm{~g}$ and the original instantaneous frequency curve is distorted. D2 spall 
deteriorates the signatures of instantaneous frequency even more, outputting an average instantaneous frequency amplitude of $15 \mathrm{~g}$. The enhanced EMD associated with TKEO effectively reveals the hidden frequency modulation of the $1^{\text {st }}$ harmonic of GMF under the influence of spalling.

In conclusion, the spall generates modulation which alters the vibration signatures. The results of amplitude demodulation, spectra analysis and CWT indicate the increase of spectral energy of the $2^{\text {nd }}$ harmonic of GMF while the magnitude of the $1^{\text {st }}$ harmonic of GMF is decreased due to the present of spalling. Phase demodulation, on the other hand, shows that the spall attenuates the instantaneous phase. The enhanced EMD decomposes the vibration signals into several IMFs and the applied TKEO characterizes the instantaneous frequency. The analysis suggests that the spall produces frequency modulation in the way that instantaneous frequency is enlarged. Analysis of all applied methods provides the comprehensive descriptions of modulation characteristics introduced by a spall. Therefore, the development of reliable and robust gearbox health monitoring techniques should be based on various signal processing techniques which can deliver different features of defects. 
(1) Baseline

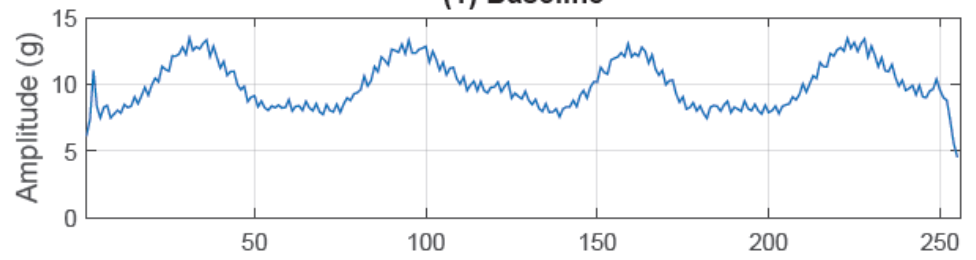

(2) D1

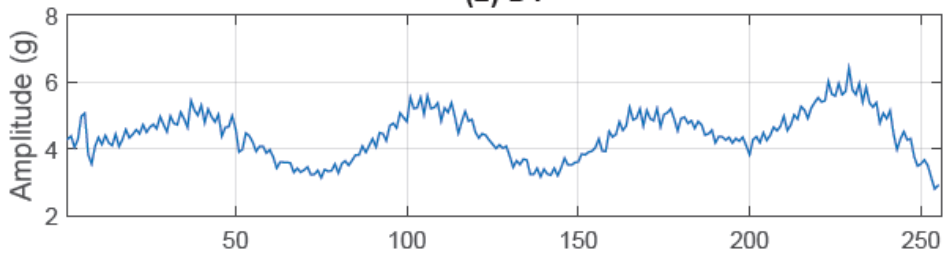

(3) D2

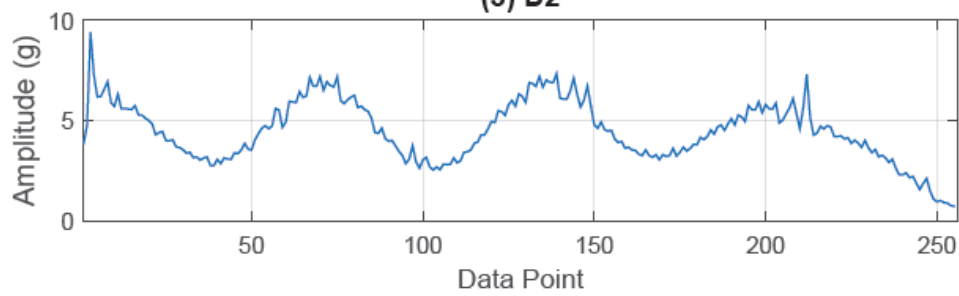

Figure 4-15 TKEO instantaneous amplitude of $\mathrm{IMF}_{2}$

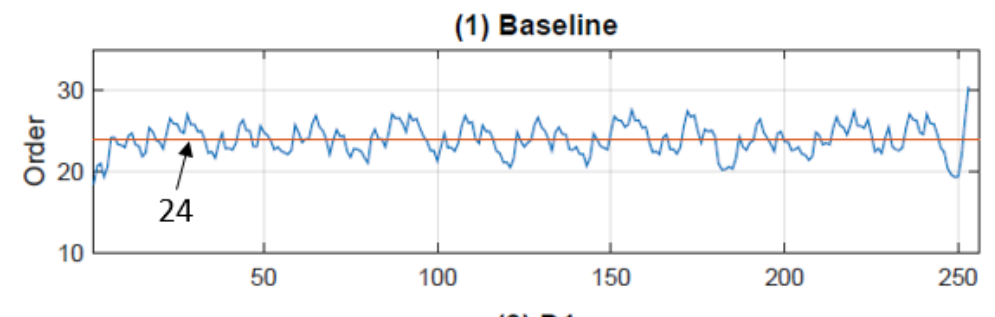

(2) D1

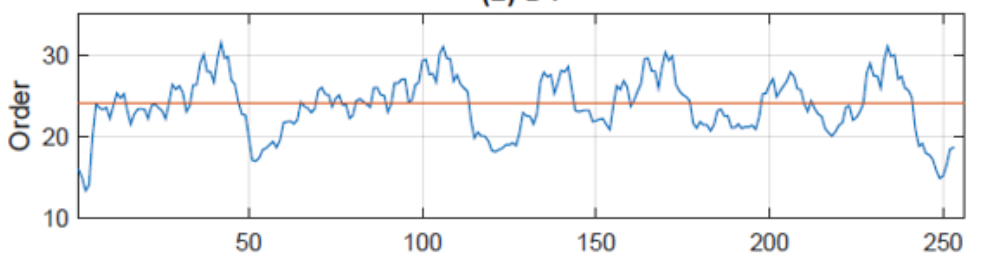

(3) D2

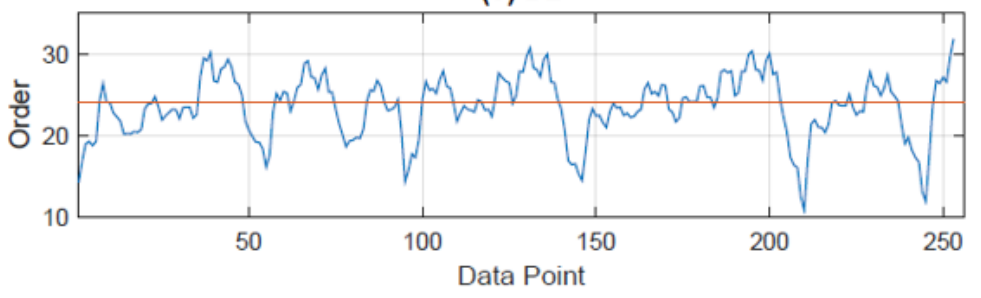

Figure 4-16 TKEO instantaneous frequency of $\mathrm{IMF}_{2}$ 


\section{Chapter 5}

\section{From the Vibration Features to the Physical State of Gear}

This chapter first provides an in-depth description of vibration features obtained from previous analysis results along with some remarkable findings. Those findings give a trace to the source of the vibration signatures. Then, a spall fault indicator is proposed based on the magnitude ratio of the $1^{\text {st }}$ and $2^{\text {nd }}$ harmonic of GMF. A discovery that bridges the vibration signatures of the digital signals and the physical state of the gear has been found and presented for the first time. The discovery leads to an exploration of a special configuration of the gearbox and provides a physical interpretation of the cause of the vibration signatures of non-defected and defect signals.

\subsection{Vibration Features}

Modulation is found in the vibration signatures from the previously discussed testing results. The baseline tests were conducted on the gearbox with normal operational teeth wear. Wear generates modulation which has a significant impact on vibration signatures. Since D1 and D2 spalls are introduced to the same worn gear, the vibrations of D1 and D2 gears are composed of modulation features due to both gear wear and spalling. 
A repeating pattern which is constructed in two relative large jumps connected by three small jumps was found in Figure 4-2 (Chapter 4). In the same figure, another hidden pattern which repeats four times during one revolution of gear F2 is discovered from the TSA signals of all gear conditions. To demonstrate the nature of the pattern, the TSA signals of all the gear conditions are divided into 4 segments respectively and the corresponding segments are overlapped in a plot for comparison. Figure 5-1 shows the plots of the overlapped TSA segments and preliminary discoveries are found based on observation. As the gear has 24 teeth, each signal segment represents the vibration signatures of every 6 teeth. From another point of view, the data between 2 extremes correspond to the vibration of one tooth since there are 12 extremes in every segment. Figure 5-1 also indicates that most areas of the four segments coincide along with small acceptable deviations. It is believed that this vibration pattern is mainly caused by modulation due to tooth wear. Segments overlap plots of D1 and D2 suggest that the influence of spalling on the characteristics of time domain signal is global instead of local, since no significant variation is observed in a single segment. A pattern that repeats four times in one revolution is also discovered from phase demodulation (Figure 4-4), wavelet map (Figure 4-6) and instantaneous amplitude of $2^{\text {nd }}$ IMF (Figure 4-15). The pattern discovered from the results of all signal processing techniques indicates that the vibration features are the direct result of the condition of the gear. Moreover, the pattern produces a frequency component that is 4 time of the shaft rotation rate, which is found in the spectrum. Besides, the findings in Figure 4-4, Figure 4-6 and Figure 4-15 converge to the same conclusion that the spall globally modulates the amplitude and phase of baseline signals, which is a special case because a gear defect usually introduces transient alteration of vibration signatures. 

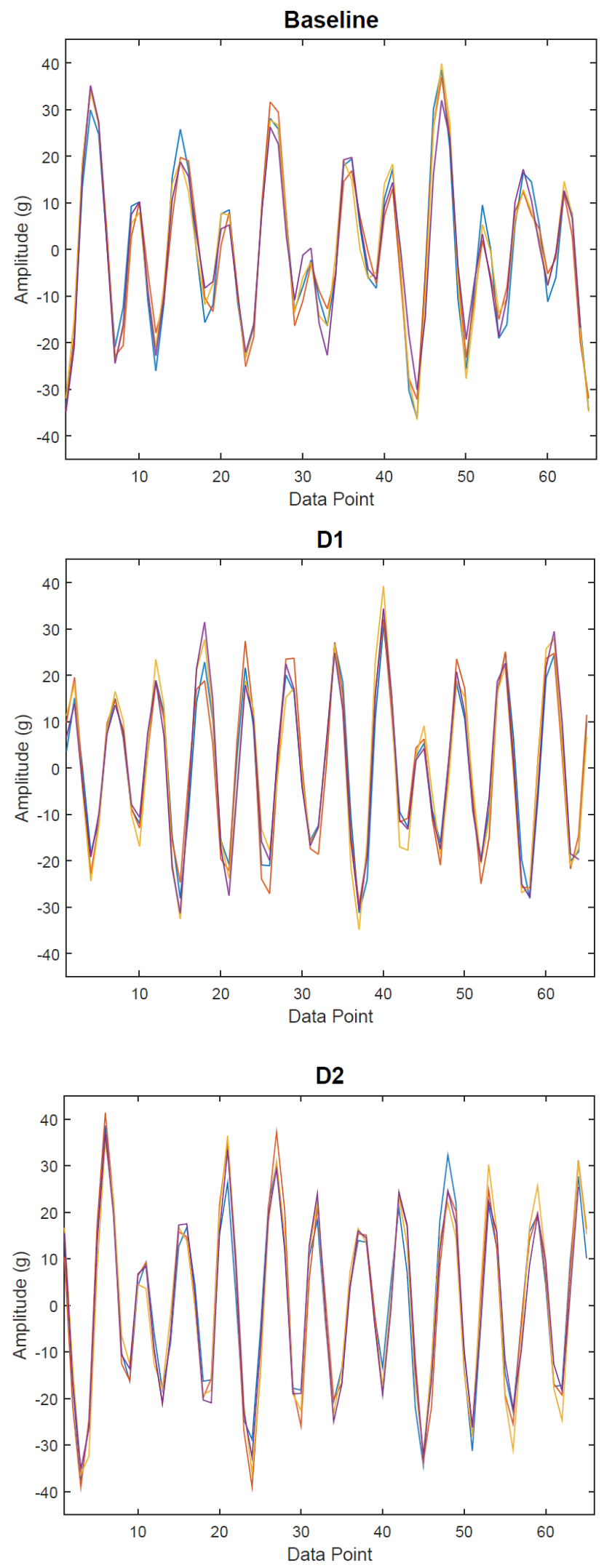

Figure 5-1 TSA signal segments overlap 1) Baseline test, 2) D1 test, 3) D2 test 


\subsection{A Proposed Spalling Defect Detection Indicator}

Frequency spectrum usually contains more features related to modulation than time domain signal, since modulation not only alters the vibration signatures of the TSA signal but also significantly affects the TSA spectra. Modulation caused by teeth wear results in sideband formation around the harmonics of GMF in a spectrum. Under most conditions, the sidebands are spaced by the amount of the shaft rotation frequency which functions as a modulation frequency in the modulation process. Figure 5-2 is the three spectra from Figure 4-5 overlapped in a plot which shows that the effect of spalling on the magnitude of sideband is negligible. It is worthy to point out that a 4 order component exists in the spectrum (Fig 5-2), which corresponds to the frequency mentioned above that is 4 times the rotation rate of the shaft. By investigating all the recorded data, it is concluded that this component has low energy and is insensitive to the load applied on the gearbox. The magnitude of the 4 order is found between 0.35 to 0.37 . This frequency is in fact called gear assembly phase frequency (GAPF) [94]. There is few document mentioning this frequency in the literature and the characteristic of GAPF will be explored in more detail in this thesis.

Chapter 4 section 4.3 addresses the issue that the spall raises the energy of the $2^{\text {nd }}$ harmonic of GMF while it lowers the magnitudes of the $1^{\text {st }}$ harmonic of GMF. In frequency domain, the spall alters the magnitude of the $1^{\text {st }}$ harmonic of GMF and the $2^{\text {nd }}$ harmonic simultaneously. In order to quantify the differences due to a spall, a magnitude ratio (MR) between the magnitudes of the $2^{\text {nd }}$ and the $1^{\text {st }}$ harmonic of GMF is introduced. The ratio is

computed as the magnitude of $2^{\text {nd }}$ harmonic of GMF over the magnitude of $1^{\text {st }}$ harmonic of GMF. The MR of all the recorded data is summarized in Table 5-1. The MR of the baseline 
data maintains below 2 whereas the MR of D1 and D2 data are all above 3. The MR of D1 data under loading condition 2 is much higher than the ratios of the other loading conditions, with an average value of 16.82, whereas the ratios of loading condition 1 and 3 are averaged to 6.15 and 5.07 respectively. A reasonable explanation is that the vibration of the target gear under loading condition 2 approaches resonance where the energy of the $1^{\text {st }}$ harmonic of GMF spreads over to the major sidebands and to the other harmonics of GMF, lowering the magnitude of itself. Apart from these high ratios, the others are consistent with the spalling size and applied load. It is interesting to point out that the MR decreases as the spall size becomes larger. The overall MR of D1 is higher than the overall MR of D2. The reason is that with a smaller spall, the magnitude of the $1^{\text {st }}$ harmonic of GMF is low. When the spall develops to a larger size, the spall generates higher vibration force and thus the magnitude of the $1^{\text {st }}$ harmonic of GMF increases. The magnitude of the $2^{\text {nd }}$ harmonic of GMF also increase but not as much as in the $1^{\text {st }}$ harmonic of GMF. So the MR decreases with a larger magnitude of the $1^{\text {st }}$ harmonic of GMF. It is concluded that spalling occurs when the MR is above 3. Defect diagnosis information can also be extracted from Table $5-1$. When the MR is computed between 3 to 5 , the spalling size is properly as large as D2 and as the MR exceeds 5, the spall has a smaller size as D1. The MR provides significant information for gearbox prognostics and health management that the development of incipient gear defects such as spalling can be monitored by the variation of MR. 


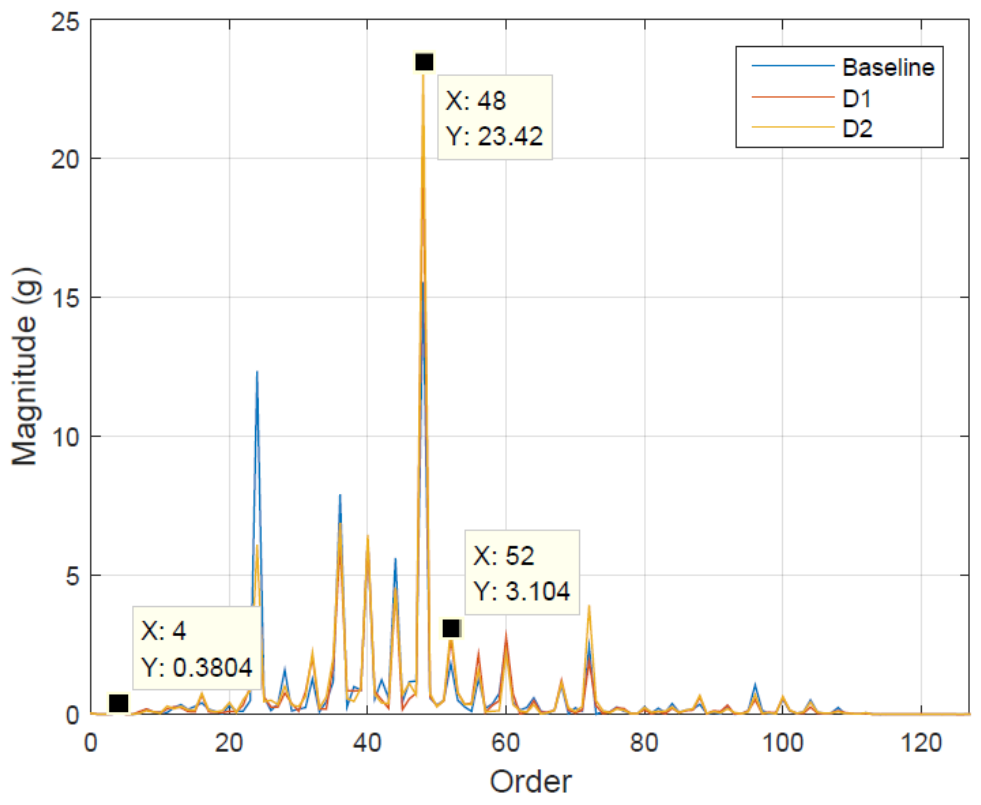

Figure 5-2 TSA spectra overlap

Table 5-1 Magnitude ratio of recorded data

\begin{tabular}{|c|c|c|c|c|}
\hline & Recording & Baseline & D1 & $\mathrm{D} 2$ \\
\hline \multirow{6}{*}{$\begin{array}{c}\text { Loading } \\
\text { condition } \\
1\end{array}$} & 1 & 1.62 & 6.43 & 3.18 \\
\hline & 2 & 1.78 & 6.23 & 3.02 \\
\hline & 3 & 1.72 & 6.52 & 3.87 \\
\hline & 4 & 1.78 & 6.63 & 3.3 \\
\hline & 5 & 1.79 & 5.24 & 5.56 \\
\hline & 6 & 1.76 & 5.86 & 3.92 \\
\hline \multirow{6}{*}{$\begin{array}{c}\text { Loading } \\
\text { condition } \\
2\end{array}$} & 1 & 1.59 & 19.83 & 5.75 \\
\hline & 2 & 1.55 & 15.52 & 4.52 \\
\hline & 3 & 1.59 & 15.1 & 4.54 \\
\hline & 4 & 1.68 & 15.86 & 5.02 \\
\hline & 5 & 1.63 & 17.21 & 6.27 \\
\hline & 6 & 1.64 & 17.39 & 5.52 \\
\hline \multirow{6}{*}{$\begin{array}{c}\text { Loading } \\
\text { condition } \\
3\end{array}$} & 1 & 1.26 & 6.12 & 3.75 \\
\hline & 2 & 1.25 & 4.48 & 3.56 \\
\hline & 3 & 1.28 & 4.66 & 3.64 \\
\hline & 4 & 1.26 & 4.41 & 3.78 \\
\hline & 5 & 1.26 & 5.4 & 3.9 \\
\hline & 6 & 1.26 & 5.35 & 3.83 \\
\hline
\end{tabular}




\subsection{Gear Assembly Phase Frequency (GAPF)}

GAPF is discovered from all the analytical data obtained from various signal processing techniques. The vibration signatures are projections of the physical condition of the gear since vibration signals are governed by the mechanism of the gearbox. GAPF is the frequency generated by a pair of mating gears whose numbers of teeth have more than one prime. In other words, the numbers of teeth of a pair of mating gears are designed to have a common factor. A pair of gears with equal numbers of teeth of the gear F2 and gear C are used to demonstrate the formation of GAPF. A schematic of gear F2 and gear C is drawn in Figure 5-3. Gear F2 has 24 teeth and gear C has 54 teeth so the largest prime number of both numbers is 6. During general gear rotation, as shown in Figure 5-3, a pair of teeth come into contact at the mesh region. A mating pair of teeth is highlighted in red and blue for gear F2 and gear C respectively. Since two gears have a prime number of 6 , the $7^{\text {th }}$ teeth of both gears apart from the mating pair in opposite direction to the rotation will mesh together. As a result, 4 teeth of gear F2 will always mesh with another specific 9 teeth of gear C. In particular, the 4 selected red teeth of gear F2 will always contact with the 9 blue teeth of gear $\mathrm{C}$ in gearbox operation, as shown in Figure 5-3. General teeth wear will develop after many hours of operation and because of the common prime number of teeth of the two gears, similar wear pattern will appear on the 4 red teeth and the 9 blue teeth. The wear on those 4 red teeth and 9 blue teeth will aggravate as the gearbox continues to operate. In consequence, two GAPFs occur in the vibration signature of the gearbox, which is equal to the one-sixth of the rotation frequency of the corresponding gear. Specifically, the GAPF of gear F2 expressed in terms of order is 4 order while the GAPF of gear $\mathrm{C}$ is 9 order. 


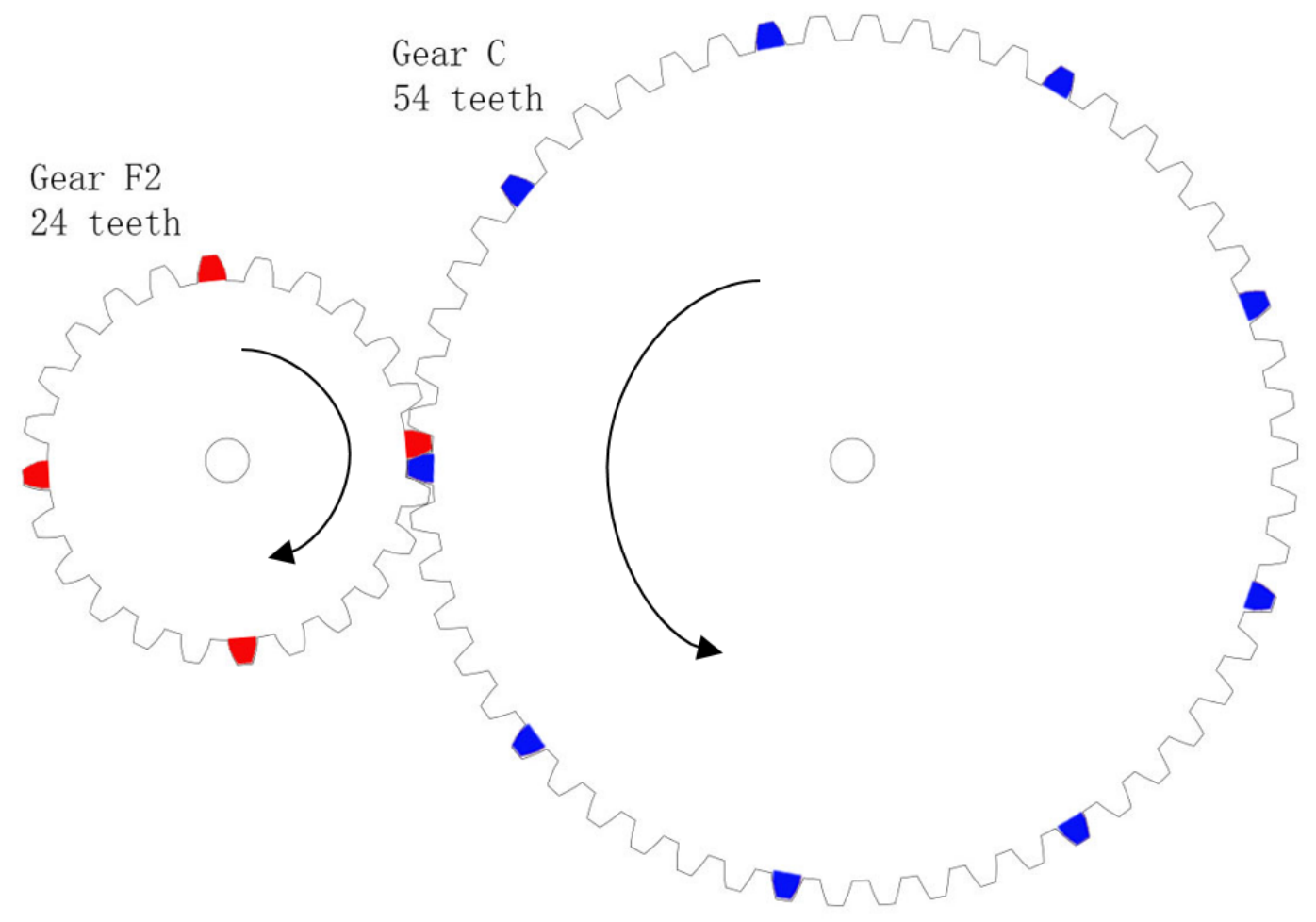

Figure 5-3 A schematic of gear F2 and gear C 


\subsection{Gear Wear Pattern}

In order to understand the source of the unique vibration signatures of gear F2, the gear was removed from the gearbox for a physical examination. The gear was examined under microscope and the pictures of each tooth were taken for analysis, as illustrated in Figure 5-4. As it can be seen, the wear pattern of teeth meets the general description: teeth wear patterns usually appear on both sides of the pitch line due to pure rolling action at the pitch line and sliding action on both sides. To clarify, the upper part of a tooth in a picture is near the tip while the lower part is close to the root of the gear. It is worthy to point out that the \#3 tooth is the defected tooth with the D2 spall. Through observation, the wear marks separated by pitch line are similar for all the teeth except the \#1, \#7, \#13 and \#19 tooth whose wear near dedendum is severer than the wear on the addendum. In fact, based on the shape of the lower wear pattern of these 4 teeth, the damage mode is scoring which appears as scratch lines in the direction of sliding. The cause of scoring is mainly owing to the mating gear $\mathrm{C}$. Before this research, other experiments were conducted to detect tooth root crack [78]. In this project, a $2.5 \mathrm{~mm}$ depth root rack was artificially introduced to the root of a tooth of gear C, as shown in Figure 5-5. During the tooth crack experiments, the gearbox was continuously operated for more than 100 hours until the tooth was broken. For the spalling research project, this gear $\mathrm{C}$ is replaced while other gears are retained. Therefore, the scoring of the 4 teeth is introduced due to the cracked tooth of gear C. In detail, the mating motion of a pair of teeth follows the action of sliding near addendum when two teeth are entering meshing, rolling at the pitch line during meshing and sliding near dedendum when they are leaving meshing. With the presence of a crack, the stiffness of a tooth is dramatically reduced and in consequence, the damaged tooth is easily bent by 
contact force and ultimately substantial tooth deflection is generated. The deflection occurs during the sliding contact of two teeth when they are leaving the mesh region but not before that moment because of the reaction time. The combined actions of tooth deflection and sliding scratch the surface near dedendum and lead to scoring formation. Due to the principle of GAPF, this cracked tooth will damage 4 evenly spaced teeth of the 24 teeth of gear F2. 


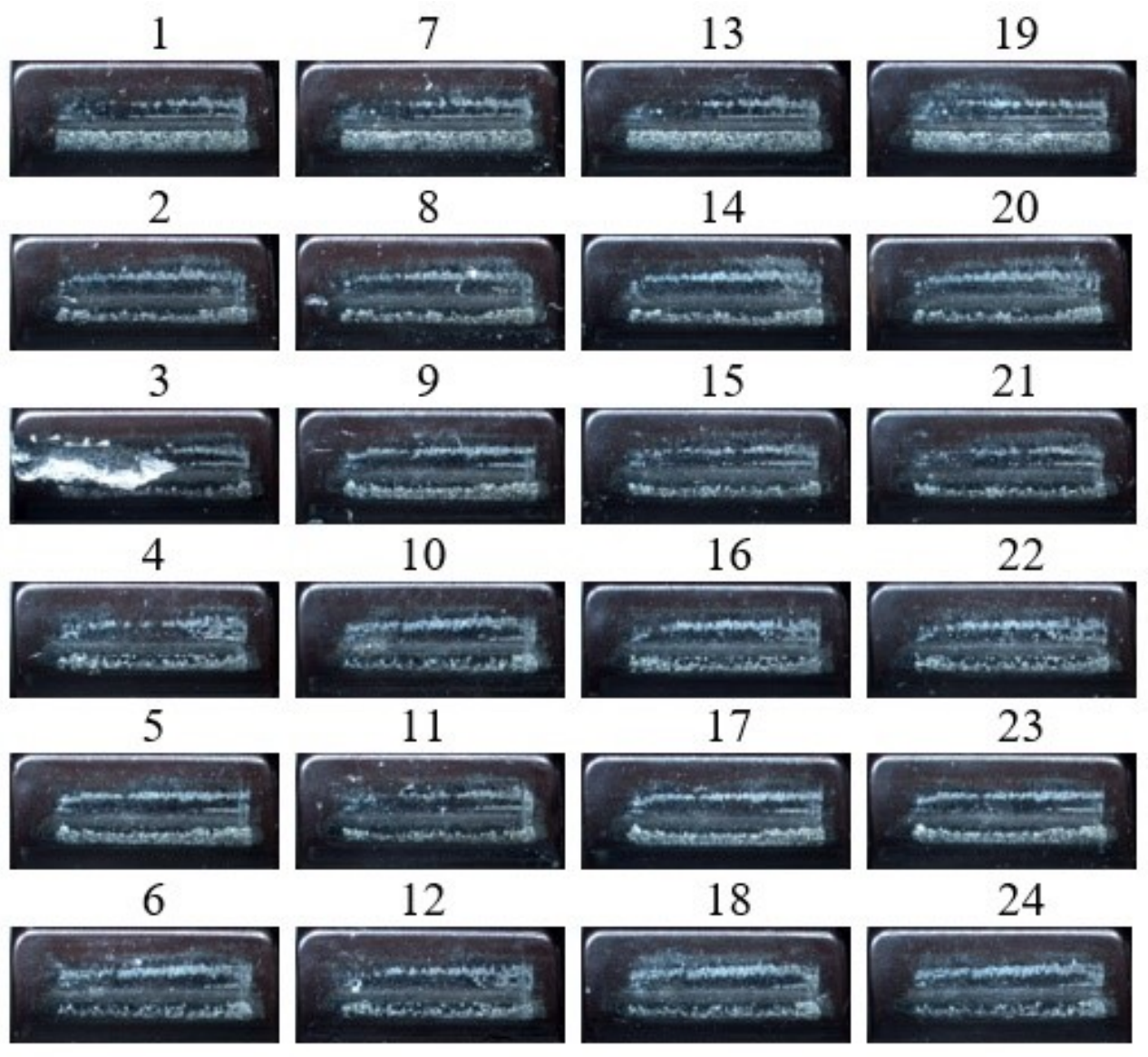

Figure 5-4 The conditions of the 24 teeth of gear F2.

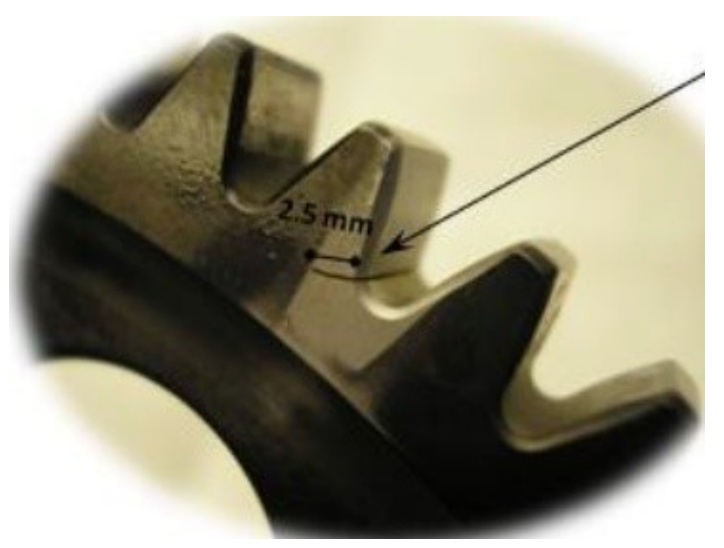

Figure 5-5 Gear C root crack [78] . 


\subsection{Vibration Signatures as Results of Gear Physical State}

The TSA accurately estimates the vibration signatures of gear F2 by removing features of the other components. It provides a close representation of vibrations of gear F2 in one revolution. Several techniques have been utilized to explore the characteristics of TSA signals obtained from different gear conditions. The 4 order component, as known as GAPF of gear F2 is continually discovered from analytic results, which include the TSA signals, amplitude and phase demodulation of TSA signals, TSA spectra and the $2^{\text {nd }}$ IMF of EMD. The GAPF is originated from the repetitive wear patterns of gear F2 which modulates dominant harmonics of GMF. In general application, a pair of gears is usually designed to have only one prime number so that all teeth of the gears are able to mate with each other to gain uniform wear on all the teeth. In such case, the harmonics of GMF are solely modulated by the rotation frequencies of both gears and the sidebands resulted from modulation are spaced by 1 order in a TSA spectrum. In this thesis, on the opposite, the sidebands are spaced by 4 orders, indicating that the 4 teeth with severe scoring produce the modulation of gear F2 in the process of teeth meshing. Analytic results from all the defect detection techniques demonstrate that a spall changes the gearmesh stiffness in a way that it excites the gearbox structure and produces modulation. Normally, a local defect such as spalling produces impulsive excitation force which changes the local characteristics of vibration signal. However, the results suggest that the spall causes global modulation effect instead of local features alternation, which is a unique case. Unfortunately, the gearbox system is complex and it is challenging to provide a persuasive explanation for this conclusion without further knowledge. 


\section{Chapter 6}

\section{Conclusion and future work}

The development of gearbox fault detection techniques is a continuously popular topic in the field of machinery condition monitoring. This thesis focuses on the development of gear spalling detection with vibration analysis. First, a complex testing system built based on an accessory gearbox of a GE J85 engine was introduced. Second, a spallwith similar shape and size to a natural formed spall is manually introduced to a pinion of the gearbox. Experiments were conducted on three different scenarios of gear health condition, which are baseline, D1 and D2 test. Vibration signals acquired from those experiments were used to develop fault detection techniques for gear spalling diagnosis.

Four well developed signal processing techniques are applied to vibration signals. TSA has the capability of background noise elimination and gears vibration pattern isolation and it is used as a preprocessed tool. RMS of TSA signals could be used as an initial indication of gearbox abnormal activity which leads to the pursue of further fault diagnosis with advanced techniques. A distinct pattern which repeats four times during one revolution of gear F2 is found from the TSA signals and the discovery is a beginning of a significant finding in gearbox fault diagnosis. Demodulation technique uncovers the features of modulated gear vibration signals due to defects. Amplitude demodulation gives an estimation of magnitude power change of the sidebands and the results are consistent with 
TSA signal analysis, in which the spall increases the amplitude of overall vibration. Phase demodulation describes the instantaneous phase change of major sidebands in the entire frequency band. The results show that the spall generates a phase function which has opposite direction to the original phase and causes phase reduction. Spectrum provides the best representation of frequency components alternation. TSA spectra indicate that spalling increases the spectral power of the $2^{\text {nd }}$ harmonic of GMF while it attenuates the spectral power of the fundamental GMF. A fault indicator, MR, is proposed based on the ratio of the power of the $2^{\text {nd }}$ and the $1^{\text {st }}$ harmonics of GMF. It is concluded that the MR can be used to monitor the development of spalling.

The extraction of local characteristics of vibration signal has been preferably focused in recent machinery condition monitoring. CWT is one of the early developed techniques that provides time-frequency representation of vibration signatures. One of many advantages of CWT is the balanced and optimized time-frequency resolution that offers fine frequency resolution in low-frequency band and good time resolution in high-frequency band. The comparison of wavelet maps among baseline, D1 and D2 TSA signals indicates that spalling decreases the energy of the fundamental GMF and increases the energy of the $2^{\text {nd }}$ GMF, which is consistent with the result from spectrum analysis. It also suggests that spalling alters the global characteristics of vibration signatures. CWT, however, usually produces redundancy due to substantial overlap moving filters and is not sensitive to the vibration signals which are less correlated to the nature of selected mother wavelet. On the other hand, CWT is not self-adaptive. An enhanced EMD is developed in this thesis to overcome these problems. The enhanced EMD decomposes a TSA signal into several IMFs, which are monocomponents. The effectiveness of the enhanced EMD associated 
with TKEO on signal demodulation has been proven with an illustration. The technique is then used to obtain the instantaneous amplitude and frequency of baseline and defected TSA signals. The findings of instantaneous amplitude are consistent with the results from spectrum analysis and wavelet map. Additionally, the outcome of EMD-TKEO reveals the frequency modulated information and the technique is superior to the other described techniques. The results demonstrate that spalling increases the frequency modulation of the fundamental GMF. It is concluded that each of these four techniques will be able to provide partial informative diagnosis of spalling damage, and it is suggested that they can be integrated as collaborative tools for more detailed defect symptom interpretation.

By investigating the vibration signatures obtained from all the methods, the GAPF is discovered in the signals. This frequency component is a result of the special design of gear parameters. Furthermore, this frequency component leads to the exploration of special gear teeth wear profile which is the source of the vibration signatures being found. This thesis offers detailed interpretation between the vibration signatures and the physical state of gear. 
Future research work could be conducted in the following subjects:

1. More experiments can be conducted to obtain further knowledge of the mechanism of the gearbox vibration.

2. Fault detection techniques for sensor in various locations could be developed for sensor classification.

3. More gear defect severities, various fault types and multiple fault gear system could also be studied to achieve comprehensive gear fault classification.

4. The spalling diagnosis could be extended to the investigation of gear fault prognosis. 


\section{References}

[1] W. Wang, "Rotary machinery health condition monitoring, fault diagnosis and prognosis," University of Waterloo, Waterloo, 2002.

[2] E. E. Shipley, "Gear Failures," 7 December 1967. [Online]. Available: http://www.xtek.com/pdf/wp-gear-failures.pdf. [Accessed 2504 2016].

[3] Y. Ding and N. F. Rieger, "Spalling formation mechanism for gears," Wear, vol. 254, no. 12, pp. 1307-1317, 2003.

[4] R. B. Randall, Vibration-Based Condition Monitoring, Chichester, England: John Wiley \& Sons, 2001.

[5] M. Åkerblom, "Gear noise and vibration - a Literature survey," Volvo Construction Equipment, Eskilstuna, 2001.

[6] S. Du, R. Randall and D. Kelly, "Modelling of spur gear mesh stiffness and static transmission error," Proceedings of the Institution of Mechanical Engineers Part C Journal of Mechanical Engineering Science, vol. 212, no. 4, pp. 287-297, 1998.

[7] A. Meisingseth, "Demodulation techniques in gearbox diagnostics," Uppsala University, Uppsala, 2012.

[8] Q. W. Wang, F. Ismail and M. F. Golnaraghi, "Assessment of gear damage monitoring techniques using vibration measurements," Mechanical Systems and Signal Processing, vol. 15, no. 5, pp. 905-922, 2001.

[9] M. Inalpolat and A. Kahraman, "A theoretical and experimental investigation of modulation sidebands of planetary gear sets," Journal of Sound and Vibration, vol. 
323, no. 3-5, pp. 677-696, 2009.

[10] Y. Li, K. Ding, G. He and H. Lin, "Vibration mechanisms of spur gear pair in healthy and fault states," Mechanical Systems and Signal Processing, 2016.

[11] P. T.R., S. K. and M. K., "Assessment of sideband energy ratio technique in detection of wind turbine gear defects," Case Studies in Mechanical Systems and Signal Processing, vol. 2, pp. 1-11, 2015.

[12] A. Jia and I. Howard, "Comparison of localised spalling and crack damage from dynamic modelling of spur gear vibrations," Mechanical Systems and Signal Processing, vol. 20, no. 2, pp. 332-349, 2006.

[13] F. Chaari, W. Baccar, M. S. Abbes and M. Haddar, "Effect of spalling or tooth breakage on gearmesh stiffness and dynamic response of a one-stage spur gear transmission," European Journal of Mechanics A/Solids, vol. 27, no. 4, pp. 691$705,2008$.

[14] R. Ma, Y. Chen and Q. Cao, "Research on dynamics and fault mechanism of spur gear pair with spalling defect," Journal of Sound and Vibration, vol. 331, no. 9, pp. 2097-2109, 2012.

[15] R. Ma and Y. Chen, "Research on the dynamic mechanism of the gear system with local crack and spalling failure," Engineering Failure Analysis, vol. 26, pp. 12-20, 2012.

[16] S. Abouel-seoud, L. Ahmed and M. Khalil, "An Experimental Study on the Diagnostic Capability of Vibration Analysis for Wind Turbine Planetary Gearbox," International Journal of Modern Engineering Research, vol. 2, no. 3, pp. 667-675, 
[17] B. D. Forrester, "Advanced Vibration Analysis Techniques for Fault Detection and Diagnosis in Geared Transmission Systems," A thesis submitted for examination for the degree of Doctor of Philosophy, Melbourne, Australia, 1996.

[18] P. D. Samuel and D. J. Pines, "A review of vibration-based techniques for helicopter transmission diagnostics," Journal of Sound and Vibration, vol. 282, no. $1-2$, pp. 475-508, 2005.

[19] H. J. Decker, "Crack Detection for Aerospace Quality Spur Gears," NASA, Cleveland, 2002.

[20] C. Pachaud, R. Salvetat and C. Fray, "Crest factor and kurtosis contributions to identify defects inducing periodical impulsive forces," Mechanical Systems and Signal Processing, vol. 11, no. 6, pp. 903-916, 1997.

[21] P. McFadden, "A technique for calculating the time domain averages of the vibration of the individual planet gears and the sun gear in an epicyclic gearbox," Journal of Sound and Vibration, vol. 144, no. 1, pp. 163-172, 1991.

[22] L. Zhu, H. Ding and X. Zhu, "Synchronous averaging of time-frequency distribution with application to machine condition monitoring," Journal of Vibration and Acoustics, vol. 129, no. 4, pp. 441-447, 2007.

[23] P. McFadden, "Interpolation techniques for time domain averaging of gear vibration," Mechanical Systems and Signal Processing, vol. 3, no. 1, pp. 87-97, 1989.

[24] P. McFadden, "A revised model for the extraction of periodic waveforms by time 
domain averaging," Mechanical Systems and Signal Processing, vol. 1, no. 1, pp. 83-95, 1987.

[25] E. B. Halim, M. S. Choudhury, S. L. Shah and M. J. Zuo, "Time domain averaging across all scales: A novel method for detection of gearbox faults," Mechanical Systems and Signal Processing, vol. 22, no. 2, pp. 261-278, 2008.

[26] K. McClintic, M. Lebold, K. Maynard, C. Byington and R. Campbell, "Residual and difference feature analysis with transitional gearbox data," in Proceedings of the 54th Meeting of the Society for Machinery Failure Prevention Technology, Virginia Beach, 2000.

[27] E. Choy, S. Huang, J. Zakrajsek, R. Handschuh and D. Townsend, "Vibration signature analysis of a faulted gear transmission system," NASA Technical Memorandum, Cleveland, 1994.

[28] X. M. V. Wang, "Autoregressive model-based gear shaft fault diagnosis using the kolmogorov-smirnovtest," Journal of Sound and Vibration, vol. 327, no. 3-5, p. 413-423, 2009.

[29] H. Endo and R. Randall, "Enhancement of autoregressive model based gear tooth fault detection technique by the use of minimum entropy deconvolution filter," Mechanical Systems and Signal Processing, vol. 21, no. 2, pp. 906-919, 2007.

[30] F. Combet and L. Gelman, "Optimal filtering of gear signals for early damage detection based on the spectral kurtosis," Mechanical SystemsandSignalProcessing, vol. 23, no. 3, pp. 652-668, 2009.

[31] P. D. McFadden, "Determining the location of a fatigue crack in a gear from the 
phase of the change in the meshing vibration," Mechanical Systems and Signal Processing, vol. 2, no. 4, pp. 403-409, 1988.

[32] H. Chen and M. J. Zuo, "Demodulation of gear vibration signals for fault detection," in Reliability, Maintainability and Safety, Chengdu, China, 2009.

[33] W. Wang, "Early detection of gear tooth cracking using the resonance demodulation technique," Mechanical Systems and Signal Processing, vol. 15, no. 5, pp. 887-903, 2001.

[34] S. Braun, Mechanical signature analysis: theory and applications, Orlando, London: Academic Press, 1986.

[35] P. McFadden, "Low frequency vibration generated by gear tooth impacts," NDT International, vol. 18, no. 5, p. 1985, 279-282.

[36] A. El-Shafei, "Gearbox fault diagnosis," RITEC, Cairo, Egypt, 2005.

[37] G. Dalpiaz, A. Rivola and R. Rubini, "Effectiveness and Sensitivity of Vibration Processing Techniques for Local Fault Detection in Gears," Mechanical Systems and Signal Processing, vol. 14, no. 3, pp. 387-412, 2000.

[38] G. Diwakar, M. R. S. Satyanarayana and P. R. Kumar, "Detection of Gear fault using vibration analysis," International Journal of Emerging Technology and Advanced Engineering, vol. 2, no. 9, pp. 2250-2459, 2012.

[39] A. V. Barkov and N. A. Barkova, "Diagnostics of gearing and geared couplings using envelope spectrum methods," VibroAcoustical Systems and Technologies, Saint-Petersburg, Russia.

[40] B. Bogert, M. Healy and J. Tukey, "The frequency analysis oftime series ofechoes: 
cepstrum pseudoautocovariance," in Proceedings ofthe Symposium on Time Series Analysis, Wiley, New York, 1963.

[41] R. B. Randall, "A history of cepstrum analysis and its application to mechanical problems," in Conference Surveillance, Chartres, Frence, 2013.

[42] R. B. Randall, "Cepstrum analysis and gearbox fault diagnosis," Brüel and Kjær Application Note, Copenhagen, Denmark, 1973.

[43] M. E. Morsy and G. Achtenová, "Vehicle gearbox fault diagnosis based on cepstrum analysis," International Journal of Mechanical, vol. 8, no. 9, pp. 1577$1583,2014$.

[44] M. E. Badaoui, J. Antoni, F. Guillet and J. Daniere, "Use of the moving cepstrum integral to detect and localise tooth spalls in gears," Mechanical Systems and Signal Processing, vol. 15, no. 5, p. 873-885, 2001.

[45] M. E. Badaoui, F. Guillet and J. Daniere, "New applications of the real cepstrum to gear signals, including definition of a robust fault indicator," Mechanical Systems and Signal Processing, vol. 18, no. 5, p. 1031-1046, 2004.

[46] D. Gabor, "Theory of communication. Part 1: The analysis of information," The Journal of the Institution of Electrical Engineers, vol. 93, no. 26, pp. 429-457, 1946.

[47] R. X. Gao and R. Yan, "From Fourier Transform to Wavelet Transform: A Historical Perspective," in Wavelets, New York, Springer US, 2011, pp. 17-32.

[48] M. L. A. e. T. M. Safizadeh, "Gear fault diagnosis using time frequency methods," in Proceeding of the 20th seminar on machinery vibration, Québec, 2002. 
[49] M. Mosher, A. H. Pryor and D. G. Lewicki, "Detailed vibration analysis of pinion gear with time-frequency methods," Ames Research Center, Moffett Field, California, 2003.

[50] R. Polikar, "The Wavelet Tutorial: Fundamental Concepts and an Overview of the Wavelet Theory," [Online]. Available: http://person.hst.aau.dk/enk/ST8/wavelet_tutotial.pdf. [Accessed 2503 2014].

[51] L. Debnath, "Brief Historical Introduction to Wavelet Transforms," International Journal of Mathematical Education in Science and Technology, vol. 29, no. 5, pp. 677-688, 1998.

[52] W. Staszewski and G. Tomlinson, "Application of the wavelet transform to fault detection in a spur gear," Mechanical Systems and Signal Processing, vol. 8, no. 3, pp. 289-307, 1994.

[53] W. M. P. D. Wang, "Application of orthogonal wavelets to early gear damage detection," Mechanical Systems and Signal Processing, vol. 9, no. 5, pp. 497-507, 1995.

[54] W. Wang and P. McFadden, "Application of wavelets to gearbox vibration signals for fault detection," Journal of Sound and Vibration, vol. 192, no. 5, pp. 927-939, 1996.

[55] D. Boulahbal, M. F. Golnaraghi and F. Ismail, "Amplitude and phase wavelet maps for the detection of cracks in geared systems," Mechanical Systems and Signal Processing, vol. 13, no. 3, pp. 423-436, 1999.

[56] C. Sung, H. Tai and C. Chen, "Locating defects of a gear system by the technique 
of wavelet transform," Mechanism and Machine Theory, vol. 35, no. 8, pp. 1169$1182,2000$.

[57] H. Zheng, Z. Li and X. Chen, "Gear fault diagnosis based on continuous wavelet transform," Mechanical Systems and Signal Processing, vol. 16, no. 2, pp. 447-457, 2002.

[58] M. Jafarizadeh, R. Hassannejad, M. Ettefagh and S. Chitsaz, "Asynchronous input gear damage diagnosis using time averaging and wavelet filtering," Mechanical Systems and Signal Processing, vol. 22, no. 1, pp. 172-201, 2008.

[59] A. Blesak and J. Flasker, "Adaptive wavelet transform method to identify cracks in gears," EURASIP Journal on Advances in Signal Processing, vol. 2010, 2010.

[60] R. Yan, R. X. Gao and X. Chen, "Wavelets for fault diagnosis of rotary machines-a review with applications," Signal Processing, vol. 96, no. Part A, pp. 1-15, 2014.

[61] P. S. Addison, "The discrete wavelet transform," in The illustrated wavelet transform handbook, Edinburgh, IOP Publishing Ltd, 2002.

[62] G. Strang and T. Nguyen, Wavelets and filter banks, Wellesley: WellesleyCambridge Press, 1996.

[63] N. Saravanan and K. Ramachandran, "Incipient gear box fault diagnosis using discrete wavelet transform," Expert Systems with Applications, vol. 37, no. 6, p. 4168-4181, 2010.

[64] Z. Li, Z. Ma, Y. Liu and W. Teng, "Crack fault detection for a gearbox using discrete wavelet transform and an adaptive resonance theory neural network," Journal of Mechanical Engineering, vol. 61, no. 1, pp. 63-73, 2015. 
[65] I. W. Selesnick, R. G. Baraniuk and N. G. Kingsbury, "The dual-tree complex wavelet transform," IEEE Signal Processing Magazine, vol. 22, no. 6, pp. 123 $151,2005$.

[66] N. Kingsbury, "The dual-tree complex wavelet transform:a new technique for shift invariance and directional filters," IEEE Digital Signal Processing Workshop, 1998.

[67] Y. Wang, Z. He and Y. Zi, "Enhancement of signal denoising and multiple fault signatures detecting in rotating machinery using dual-tree complex wavelet transform," Mechanical Systems and Signal Processing, vol. 24, no. 1, pp. 119-137, 2010.

[68] N. E. Huang, Z. Shen and S. R. Long, "The empirical mode decomposition and the Hilbert spectrum for nonlinear and non-stationary time series analysis," Proc. $R$. Soc. Lond. A, vol. 454, no. 1971, pp. 903-995, 1998.

[69] S. Loutridis, "Damage detection in gearsystems using empirical mode decomposition," Engineering Structures, vol. 26, no. 12, p. 1833-1841, 2004.

[70] J. Wang, G. Zhou, X. Zhao and S. Liu, "Gearbox fault diagnosis and prediction based on empirical mode decomposition scheme," in International Conference on Machine Learning and Cybernetics, Hong Kong, 2007.

[71] J. Cheng, D. Yu and Y. Yang, "The application of energy operator demodulation approach based on EMD in machinery fault diagnosis," Mechanical Systems and Signal Processing, vol. 21, no. 2, p. 668-677, 2007.

[72] W. Teng, F. Wang, K. Zhang, Y. Liu and X. Ding, "Pitting fault detection of a wind turbine gearbox using empirical mode decomposition," Journal of Mechanical 
Engineering, vol. 60, no. 1, pp. 12-20, 2014.

[73] Z. Wu and N. E. Huang, "Ensemble empirical mode decomposition: a noiseassisted data analysis method," Advances in Adaptive Data Analysis, vol. 01, no. 01, pp. 1-41, 2009.

[74] J. Lu, F. Meng, H. Shen, L. Ding and S. Bao, "Gear fault diagnoise based on ensemble empirical mode decomposition and instantaneous energy density spectrum," Applied Mechanics and Materials, vol. 142, no. 3, pp. 3-6, 2012.

[75] C. Yang and T. Wu, "Diagnostics of gear deterioration using EEMD approach and PCA process," Measurement, vol. 61, pp. 75-87, 2015.

[76] L. Zhao, D. Huang and Y. Qin, "Fault diagnosis for gearbox based on improved empirical mode decomposition," Shock and Vibration, vol. 2015, no. Article ID 215806, p. 9 pages, 2015.

[77] "Canadair CT-114 Tutor," Canadian Warplane Hertiage Museum, [Online]. Available: http://www.warplane.com/vintage-aircraft-collection/aircrafthistory.aspx?aircraftId=8. [Accessed 25 April 2016].

[78] A. Rezaeia and A. Dadouche, "Development of a Turbojet Engine Gearbox Test Rig for Prognostics and Health Management," Journal of Mechanical System and Signal Processing, vol. 33, pp. 299-311, 2012.

[79] "General Electric J85-GE-17A Turbojet Engine, Cutaway," Smithsonian National Air and Space Museum, [Online]. Available: http://airandspace.si.edu/collections/artifact.cfm?id=A19800072000. [Accessed 01 02 2016]. 
[80] A. Dadouche, A. Rezaeib, V. Wickramas, W. Dmochowskiah, J. W. Birda and F. Nitzsc, "Sensitivity of Air-Coupled Ultrasound and eddy current sensors to bearing fault detection," Tribology Transaction, vol. 51, no. 3, pp. 310-323, 2008.

[81] A. Rezaeia, A. Dadoucheb, V. Wickramas and W. Dmochowskib, "A comparison study between Acoustic sensors for bearing fault detection under different speed and load using a variety of signal processing techniques," Tribology Transaction, vol. 59, no. 2010, pp. 179-186, 2010.

[82] A. Rezaeia, "Multi-Sensor Based Framework For Gear Condition Monitoring," Queen's University, Kingston, 2013.

[83] L. Zhu, H. Ding and X. Zhu, "Synchronous Averaging of Time-Frequency Distribution With Application to Machine Condition Monitoring," Journal of Vibration and Acoustics, vol. 129, no. 4, pp. 441-447, 2007.

[84] E. Bechhoefer and M. Kingsley, "A Review of Time Synchronous Average Algorithms," in Annual Conference of the Prognostics and Health Management Society, San Diego, 2009.

[85] G. Forber, "Phase demodulation using the Hilbert transform in the frequency domain," 0701 2010. [Online]. Available: http://www.varg.unsw.edu.au/Assets/link pdfs/phase_demodulation_rev-1.pdf. [Accessed 0405 2016].

[86] H. R. Martin, "Statistical moment analysis as a means of surface damage detection," in The Systems Engineering Approach TO Mechanical Failure Prevention, 47th Meeting of MFPG, New York, 1993. 
[87] L. Jie, "An Intelligent System for Bearing Condition Monitoring," Univeristy of Waterloo, Waterloo, 2008.

[88] R. Rato, M. Ortigueira and A. Batista, "On the HHT, its problems, and some solutions," Mechanical Systems and Signal Processing, vol. 22, no. 6, pp. 1374$1394,2008$.

[89] J. F. Kaiser, "On a simple algorithm to calculate the 'energy' of a signal," in Acoustics, Speech, and Signal Processing, Albuquerque, NM, 1990.

[90] P. Maragos, J. F. Kaiser and T. F. Quatieri, "On amplitude and frequency demodulation using energy operators," IEEE Transactions on Signal Processing, vol. 41, no. 4, pp. 1532 - 1550, 1993.

[91] D. Vakman, "On the analytic signal, the Teager-Kaiser energy algorithm, and other methods for defining amplitude and frequency," IEEE Transactions on Signal Processing, vol. 41, no. 4, pp. 791 - 797, 1996.

[92] H. Liu, X. Wang and C. Lu, "Rolling bearing fault diagnosis based on LCD-TEO and multifractal detrended fluctuation analysis," Mechanical Systems and Signal Processing, Vols. 60-61, pp. 273-288, 2015.

[93] P. Maragos, J. F. Kaiser and T. F. Quatieri, "Energy separation in signal modulations with application to speech analysis," IEEE Transactions on Signal Processing, vol. 41, no. 10, pp. 3024 - 3051, 1993.

[94] Mobius Institute, "Utilizing Vibration Analysis to Detect Gearbox Faults," 2006 2014. [Online]. Available: https:/www.youtube.com/watch?v=0TH5SLghYPY. [Accessed 1205 2016]. 